\title{
In vitro investigation of trans SNARE complexes arrested between artificial membranes
}

\author{
PhD Thesis \\ in partial fulfilment of the requirements \\ for the degree "Doctor of Philosophy (PhD) \\ in the Molecular Biology Program \\ at the Georg August University Göttingen, \\ Faculty of Biology
}

submitted by

Halenur Yavuz

born in

Bandirma, Turkey 


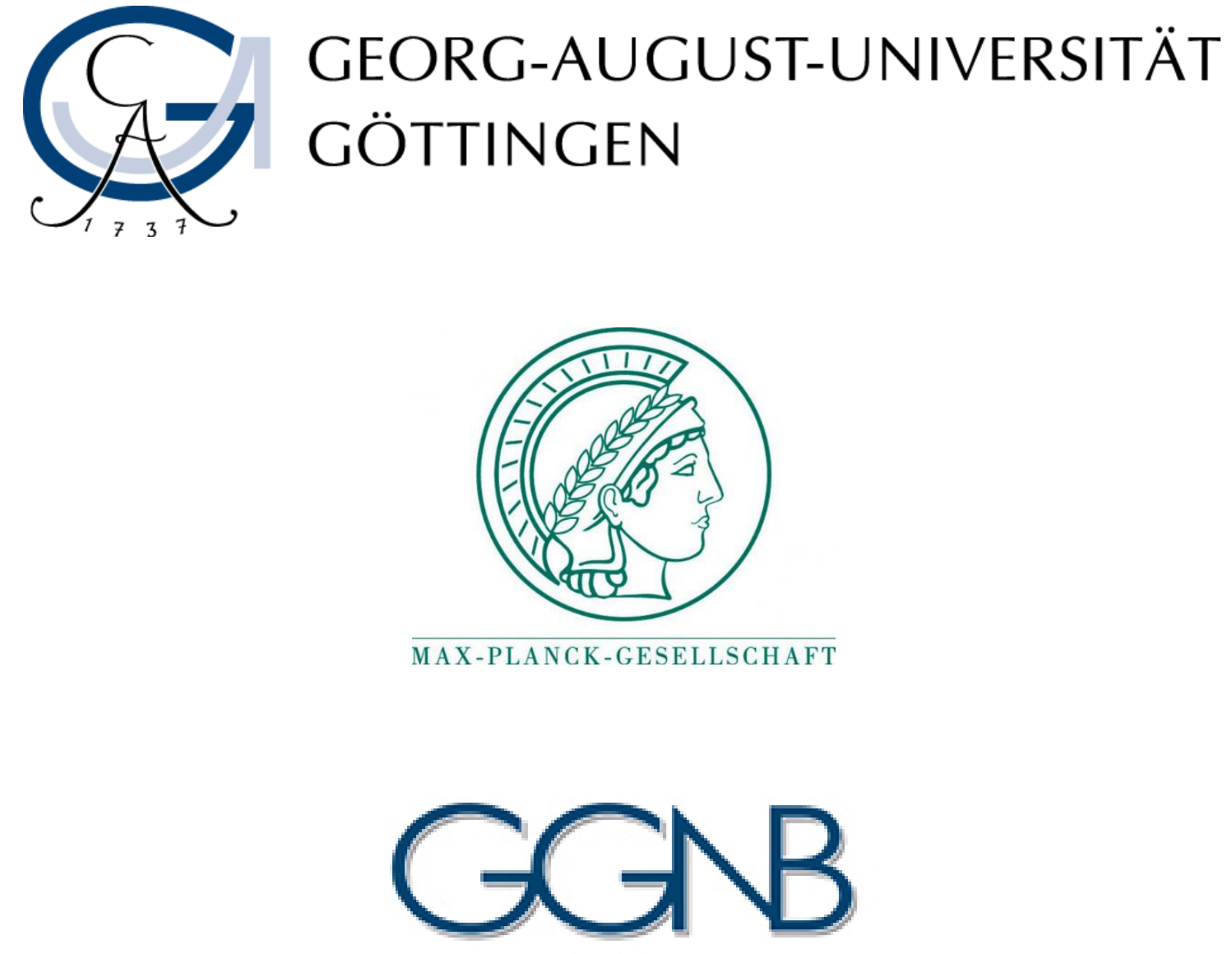

Göttingen Graduate School for Neurosciences and Molecular Biosciences

\section{Thesis Advisory Committee}

\section{Prof. Dr. Reinhard Jahn}

Department of Neurobiology, Max Planck Institute for biophysical chemistry

\section{Prof. Dr. Dirk Görlich}

Department of Cellular Logistics, Max Planck Institute for biophysical chemistry

\section{Prof. Dr. Silvio Rizzoli}

Department of Neuro- and Sensory Physiology, University Medical Center Göttingen

Date of the oral examination: 21 November, 2014 



\section{Declaration of Authorship}

Herewith I declare, that I prepared the $\mathrm{PhD}$ Thesis "In vitro investigation of trans SNARE complexes arrested between artificial membranes" on my own and with no other sources and aids than quoted.

Göttingen, 30.09.14

Halenur Yavuz 


\section{Acknowledgements}

I would like to thank Prof. Dr. Reinhard Jahn, who is a great supervisor and does wonderful things, from sharing valuable scientific ideas, establishing a distinguished international graduate school to organizing enjoyable bicycle tours! I am honored that he gave me the chance to work in his laboratory and always encouraged me to design my own experiments.

I am indebted to Dr. Matias Hernandez, who supervised me during my rotation and the first years of my doctoral studies. I am very grateful for his patience and Jedi mentoring skills, which toughened me up and set me free. I am only hoping that I could live up to his expectations from a young padawan.

I am thankful to the members of my thesis committee, Prof. Dr. Dirk Görlich and Prof. Dr. Silvio Rizzoli for their advice and feedback. I am grateful to have worked with my collaborators and the people I have had the chance to share insightful discussions: Iman Kattan, Prof. Dr. P. Jomo Walla, Dr. Dietmar Riedel, Dr. Jelger Risselada, Dr. Partho Halder, Dr. Angel Perez Lara and especially Dr. Yongsoo Park, with whom we together were amused by the mystery of how the protein, NSF worked.

I am pleased to be the part of the Department of Neurobiology, thank you all for keeping the mood always joyful! My special thanks in the department go to Ursel Ries for her excellent technical support and to our skillful managers Dr. Gottfried Mieskes, Dr. Hans Dieter Schmitt and Elisa Reckmann-Heinrich. The second team, which I was as proud to be part of was our 'MolBio' group in the greater GGNB family. I would like to thank Dr. Steffen Burkhardt, Kerstin Grüniger and the GGNB office for always being there to prepare us for Göttingen life in the best way.

I would like to thank my labmates, Julia, Matias, Esra, Momchil, Beyenech, Saskia, Caro, Dominika, Janina, Andreea for sharing with me not only their academic experience but also their personal adventures. I would also like to mention Kevser, who was always a part of those cheerful adventures. 
I want to mention the people who have been great inspiration to me: Dr. Murat Yaylaoğlu, Çalışkan ailesi, Pınar Veziroğlu, Damla Kalaycı (huzur içinde uyu), and people of the Istanbul Gezi Park.

My special thanks go to Nickias for his endless love and support. I would be lost many times without him being on the other side of the line. Every time an experiment put me up or down, he invented a new way to reset 700 kilometers to zero. Vielen Dank, Nicki! These moments were only a little part of what we have shared and will ever do happily.

The last but not the least, I am very indebted to my family and would like to dedicate this thesis to them. Sizin desteğiniz olmadan bu sayfaların hiç biri yazılamazdı. Bilim merakıma benden çok sahip çıktığınız için, her aylığınızın ilk kısmını kitapçıda harcadığımız için teşekkür ederim. Beni beni bu denli açık fikirli, mutlu yetiştiren her fedakarlığınıza binlerce kez minnetarım. Tezimi anneme, babama ve biricik kız kardeşime armağan ediyorum. 


\section{Table of Contents}

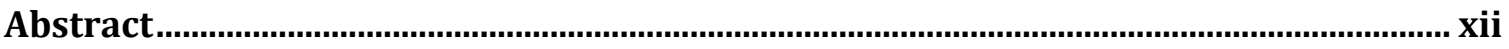

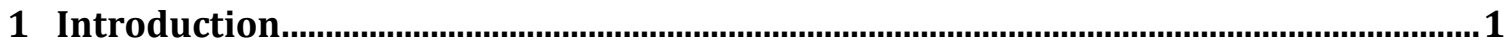

1.1 SNAREs: key members of membrane fusion machinery …....................................... 2

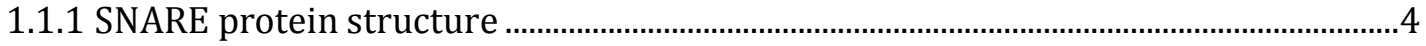

1.1.2 Structure of the fully assembled SNARE complex..............................................................

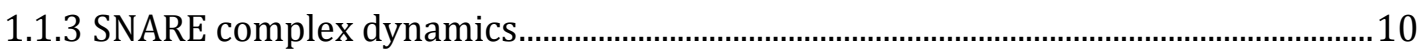

1.1.4 Proteins regulating the SNARE complex cycle.............................................................. 14

1.2 Partially assembled SNARE complexes ............................................................... 19

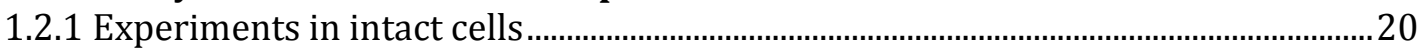

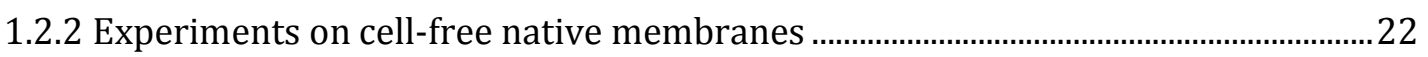

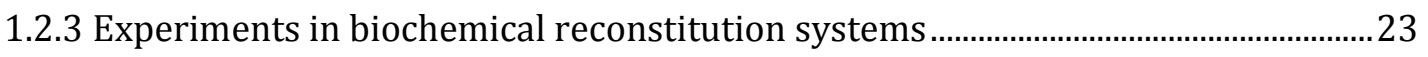

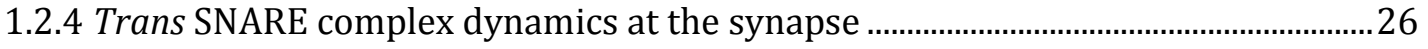

1.3 Aims of this study

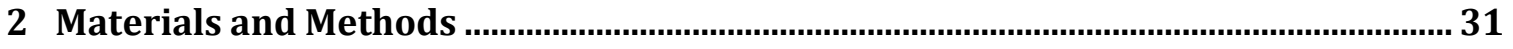

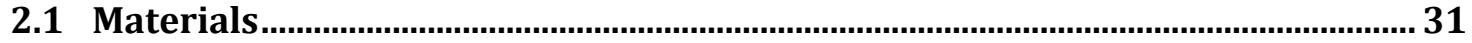

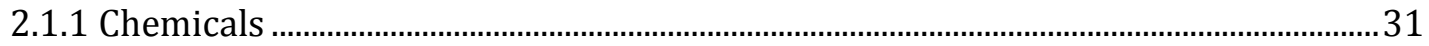

2.1.2 Buffers and media......................................................................................................... 31

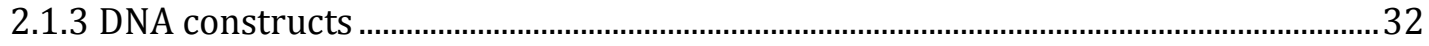

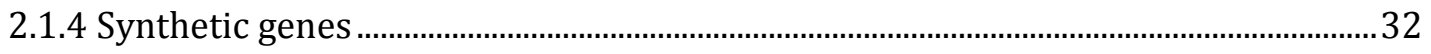

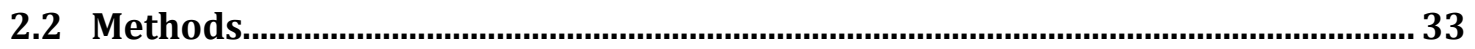

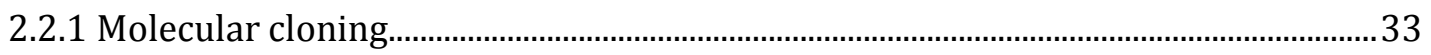

2.2.2 Protein expression and purification ................................................................................ 36

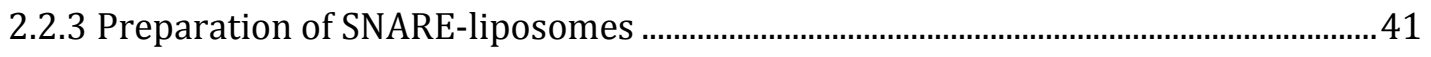

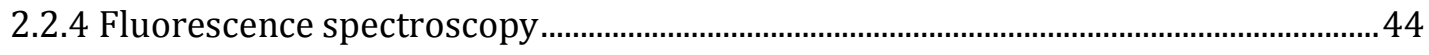

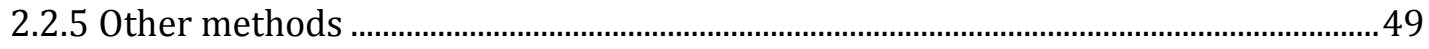

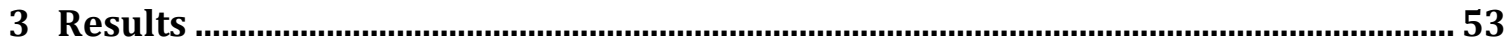

3.1 Strategies to reconstitute trans SNARE complexes in vitro.................................... 53

3.1.1 First reconstitution system: Q-SNARE complexes as acceptor sites ...........................53

3.1.2 Second reconstitution system: two different approaches for monitoring cis and

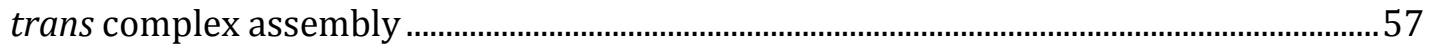

3.1.3 Final reconstitution system: using stabilized acceptor complexes.............................62 
3.2 trans SNARE complex dynamics: tightly zippering complexes...............................63

3.2.1 The deletion mutant does not facilitate large liposome fusion......................................63

3.2.2 NSF/ $\alpha$-SNAP-mediated disassembly of trans SNARE complexes ................................ 64

3.2.3 SNARE monomers do not re-assemble after trans complex disassembly................69

3.2.4 NSF/ $\alpha$-SNAP-mediated disassembly of the acceptor complexes ................................... 70

3.2.5 trans SNARE complexes are not "NSF-resistant" .............................................................. 72

3.3 trans SNARE complex dynamics: loosely zippering complexes............................ 76

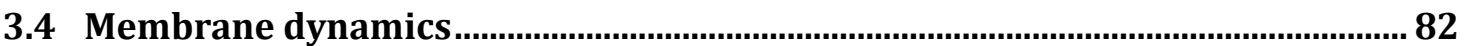

3.4.1 Do liposomes stop docking if all of the trans complexes are fully disassembled by

NSF? 83

3.4.2 What is the extent of zippering in trans SNARE complexes?

4 Discussion

4.1 Considerations for constructing an in vitro reconstitution system to study trans SNARE complexes.

4.2 Introducing a reconstitution system to study two types of trans SNARE

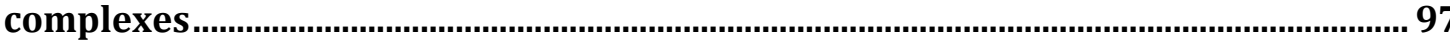

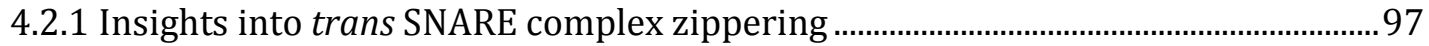

4.2.2 Insights into NSF-mediated trans SNARE complex disassembly .............................. 102

4.2.3 Irreversible docking of large liposomes ............................................................................. 104

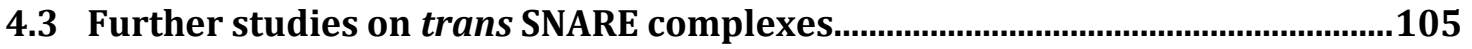

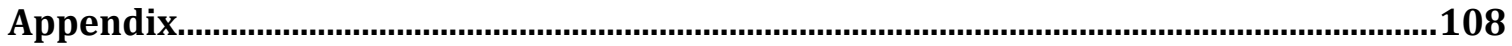

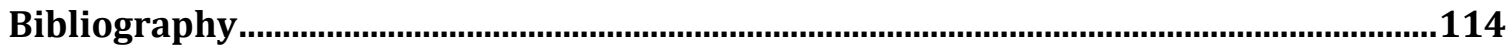

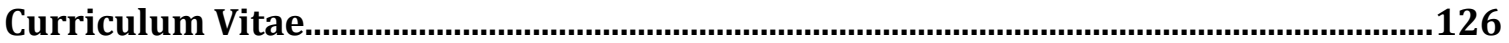




\section{List of Figures}

1.1. A simplified overview of the vesicular transport process......................................................

1.2. Domain compositions of the synaptic SNARE proteins...........................................................

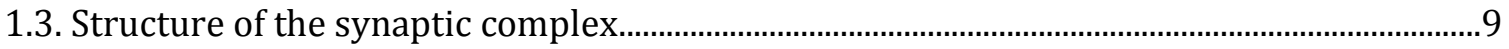

1.4. Conformational cycle of SNAREs during neuroexocytosis........................................................ 12

1.5. Two models describing the transition between priming and triggering steps .................. 18

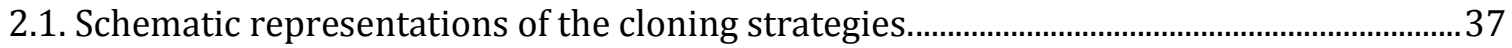

2.2. Expression and purification profile of syb (1-116) S28C, $\Delta 84$ (the deletion mutant) ..... 40

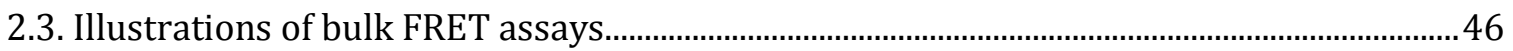

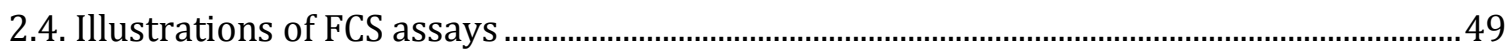

2.5. Illustration of the co-flotation assay.................................................................................. 49

3.1. Schematic representation of the first reconstitution system................................................... 54

3.2. Single labeled ternary SNARE complexes on large liposomes are disassembled...............55

3.3. Compositions of the SNARE complexes that assembled overnight remained unclear.....56

3.4. Schematic representation of the second reconstitution system...............................................58

3.5. Double labeled ternary complexes were purified \& reconstituted on large liposomes ..59

3.6. Double labeled ternary SNARE complexes are disassembled ...................................................60

3.7. Syntaxin and syb $\Delta 84 \mathrm{OG}$ were reconstituted on large liposomes successfully..................60

3.8. Trans SNARE complex dynamics in the second reconstitution system................................ 61

3.9. Schematic representation of the third reconstitution system................................................ 63

3.10. The deletion mutation prevents large liposome fusion......................................................... 64

3.11. Assembly of the trans SNARE complexes between large liposomes was monitored..... 65

3.12. NSF/ $\alpha$-SNAP disassembles trans SNARE complexes on large liposomes. ...........................67

3.13. FRET signal marks the interactions of the proteins ............................................................ 68

3.14. NSF/ $\alpha$-SNAP disassembles full-length trans SNARE complexes............................................69

3.15. SNARE monomers do not re-assemble after trans complex disassembly ........................... 70

3.16. SN25 dissociates off the membrane upon disassembly ........................................................... 71

3.17. Almost all of the syb $\Delta 84$ monomers assemble into trans SNARE complexes .................. 73

3.18. Incorporating TeNT to the NSF-driven trans complex disassembly reaction .................75

3.19. Large syb AA liposomes do not fuse with large acceptor liposomes. ..................................77

3.20. Trans SNARE complex assembly reactions monitored via the FRET-based assay..........78

3.21. Displacement of labeled syb (49-96) compared via fluorescence anisotropy .................79 
3.22. Trans SNARE complex assembly reactions monitored via fluorescence anisotropy .....81

3.23. Some of the syb AA monomers assemble into trans SNARE complexes.............................. 82

3.24. Large liposomes stay docked after trans complexes are disassembled..............................85

3.25. NSF/ $\alpha$-SNAP affects SNARE-dependent docking of syb $\Delta 84$ large liposomes ..................87

3.26. NSF/ $\alpha$-SNAP affects SNARE-dependent docking of syb AA large liposomes.....................89

3.27. Not all of the acceptor complexes are associated with synaptobrevin ...............................91

3.28. Syb wt (1-96) competes with the assembling trans SNARE complexes .............................92

4.1. Schematic representation of the modifications to the reconstitution system ................... 106 


\section{List of Tables}

2.1. List of buffers and media used in this study .......................................................................... 31

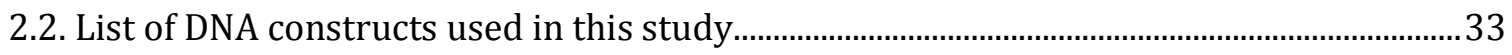

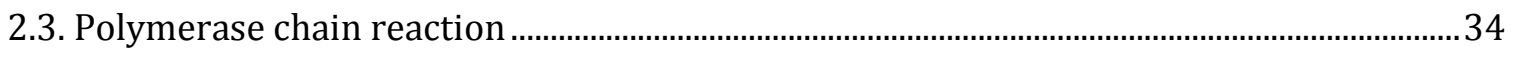

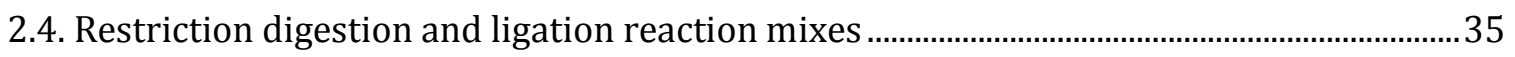

2.5. Composition of a lipid mix for large liposome preparations..................................................... 42

2.6. Reconstitution mix to prepare synaptobrevin liposomes ............................................................ 43

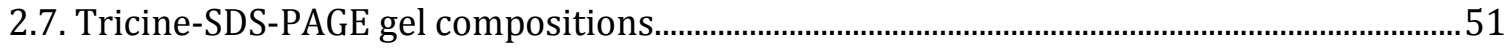




\section{List of Abbreviations}

$\begin{array}{ll}\alpha \text {-SNAP } & \text { N-ethylmaleimide-sensitive factor attachment protein alpha } \\ \text { ATP } & \text { adenosine triphosphate } \\ \mathrm{CaCl}_{2} & \text { calcium chloride } \\ \text { chol } & \text { cholesterol } \\ \text { ddH }_{2} 0 & \text { double distilled water } \\ \text { DTT } & \text { dithiothreitol } \\ \text { EDTA } & \text { ethylenediaminetetraacetic acid } \\ \text { f.c. } & \text { final concentration } \\ \text { FCS } & \text { fluorescence correlation spectroscopy } \\ \text { FCCS } & \text { fluorescence cross-correlation spectroscopy } \\ \text { FRET } & \text { Förster resonance energy transfer } \\ \text { HEPES } & \text { 2-[4-(2-hydroxyethyl) piperazin-1-yl] ethane sulfonic acid } \\ \text { HCl } & \text { hydrochloric acid } \\ \text { HRP } & \text { horse radish peroxidase } \\ \text { IMPACT } & \text { intein mediated purification with an affinity chitin-binding tag } \\ \text { IPTG } & \text { isopropyl } \beta \text {-D-1-thiogalactopyranoside } \\ \text { KAc } & \text { potassium acetate } \\ \text { KCl } & \text { potassium chloride } \\ \text { KGlu } & \text { potassium glutamate } \\ \text { LB } & \text { lysogeny broth } \\ \text { mAU } & \text { milli absorbance units } \\ \text { mbar } & \text { millibar } \\ \text { MgCl } 2 & \text { magnesium chloride } \\ \text { min } & \text { minutes } \\ \text { mS/cm } & \text { milli siemens per centimeter } \\ \text { MW } & \text { molecular weight } \\ \text { NaCl } & \text { sodium chloride } \\ \text { NBD } & \text { nitrobenzoxadiazole } \\ \end{array}$




$\begin{array}{ll}\text { NBD-PS } & \text { 1,2-dioleoyl-sn-glycero-3-phospho-L-serine-N- } \\ & \text { (7-nitro-2-1,3-benzoxadiazol-4-yl) } \\ \text { NEB } & \text { New England Biolabs } \\ \text { NSF } & \text { N-ethylmaleimide-sensitive factor } \\ \text { nm } & \text { nanometer } \\ \text { OD } \lambda & \text { optical density (at wavelength } \lambda \text { ) } \\ \text { og } & \text { n-octyl- } 3 \text {-D-glucoside } \\ \text { OG } & \text { Oregon Green 488 iodoacetamide } \\ \text { OG-DHPE } & \text { Oregon Green 488 1,2-Dihexadecanoyl-sn-Glycero-3-Phosphoethanolamine } \\ \text { o/n } & \text { overnight } \\ \text { PC } & \text { phosphatidylcholine } \\ \text { PCR } & \text { polymerase chain reaction } \\ \text { PE } & \text { phosphatidylethanolamine } \\ \text { PS } & \text { phosphatidylserine } \\ \text { Rho } & \text { rhodamine } \\ \text { Rho-PE } & \text { 1,2-dioleoyl-sn-glycero-3-phosphoethanolamine-N- } \\ & \text { (lissamine rhodamine B sulfonyl) } \\ \text { rpm } & \text { revolutions per minute } \\ \text { S } & \text { second } \\ \text { SN25 } & \text { SNAP-25 } \\ \text { SNARE } & \text { soluble N-ethylmaleimide-sensitive factor attachment protein receptor } \\ \text { syb } & \text { Synaptobrevin 2 } \\ \text { syx } & \text { Syntaxin 1A } \\ \text { TB } & \text { terrific broth } \\ \text { TCEP } & \text { tris (2-carboxyethyl) phosphine } \\ \text { TR } & \text { Texas Red c5 bromoacetamide } \\ \text { TR-DHPE } & \text { Texas Red 1,2-Dihexadecanoyl-sn-Glycero-3-Phosphoethanolamine } \\ \text { V } & \text { volt } \\ \text { v/v } & \text { volume/volume } \\ \text { w/v } & \text { weight/volume } \\ & \end{array}$




\section{Abstract}

The key components of the machinery that catalyzes membrane fusion include membrane-associated small proteins that are termed as soluble $\mathrm{N}$-ethylmaleimidesensitive factor attachment protein receptors (SNAREs). Calcium-triggered synaptic vesicle exocytosis is one of the most investigated SNARE-mediated fusion processes. Three synaptic SNARE proteins zipper into ternary complexes from their membranedistant $\mathrm{N}$-termini towards their membrane proximal C-termini. This assembly drives the merger of the synaptic vesicles with the pre-synaptic membrane. After the exocytosis, ternary complexes are disassembled into reactivated monomers for subsequent rounds of neurotransmitter release. This cycle includes a transient assembly intermediate that forms as the synaptic vesicle SNARE (synaptobrevin) starts zippering with the pre-synaptic plasma membrane SNAREs (syntaxin and SNAP-25). Prior to membrane fusion, partially zippered SNARE complexes are found in trans configurations with the transmembrane domains of synaptobrevin and syntaxin residing in two opposing membranes. These complexes are the substrates of various regulatory factors that control the calcium-triggered release process tightly. Current models of this regulation are based on the indirect measurements of membrane fusion probes. It was not possible so far to address the protein-protein interactions directly due to difficulties in isolating the short-lived trans SNARE complexes. This study presents a biochemically well-defined reconstitution system that captures such trans complexes successfully. SNARE zippering was artificially arrested between docking, but not-fusing large liposomes (diameter, $100 \mathrm{~nm}$ ) by using mutants of synaptobrevin. The disassembly machinery (NSF and $\alpha$-SNAP) and tetanus neurotoxin were effectively incorporated into this system. Several biochemical assays were developed utilizing these factors to study the characteristics of the trans complexes. The assembly and disassembly cycles of the trans SNARE complexes were monitored directly via fluorescently labeled proteins or indirectly via fluorescently labeled lipids. These measurements were extremely helpful in deducing the extent of SNARE zippering. Each of the two synaptobrevin mutants was 
involved in very different trans configurations depending on the region where their zippering was arrested, one being loosely and the other being tightly zippered. These advances proved this reconstitution system as an ideal medium for further investigations. It is made possible to address the key steps that regulate the progression from loose to tight zippering directly. Understanding this regulation is crucial for elucidating the step-wise mechanisms that attain high accuracy and speed in synaptic transmission. 


\section{Introduction}

Eukaryotic cells display a remarkable organization of functionally distinct membrane-enclosed compartments, organelles. Intracellular trafficking of the transport vesicles in the network of organelles assures the integrity and function of this network. Thanks to Palade and co-workers, the fundamental mechanisms of this trafficking process was explained via the vesicular transport hypothesis [1]. According to this hypothesis, transport vesicles bud from a donor compartment. They are subsequently targeted to a specific acceptor compartment, where they fuse with the acceptor membrane to deliver their cargo.

Newly synthesized secretory proteins are examples of such cargo molecules. For a successful round of secretion, the transport vesicles at their acceptor compartments must fuse successfully to the target membranes. Figure 1.1 provides a very simplified overview of the vesicular transport hypothesis with an emphasis on membrane fusion. The protein machinery mediating this key fusion step includes soluble $\mathrm{N}$ ethylmaleimide-sensitive factor attachment protein receptors (SNAREs). So far, neurotransmitter release in neurons has been one of the best studied SNAREmediated secretion processes, which was also the center of focus in this study.

The major incentive of this work was to develop a biochemical reconstitution system to study the dynamics of the neuronal SNARE proteins assembling into complexes between artificial membranes prior to their fusion. This thesis presents it with five chapters. The first chapter introduces how SNARE proteins and their regulators orchestrate membrane fusion. Particularly, the approaches that had been taken so far to arrest partially assembled SNARE complexes and why there is still a need for a new approach are explained. The second chapter describes the experimental techniques 
for purifying recombinant proteins, preparing proteo-liposomes and monitoring these probes via fluorescence spectroscopy. The third chapter reports on the results obtained throughout this study in four sections. Its first section compares different methods that were applied to develop a suiting biochemical reconstitution system for the purpose of the study. The observations made using the newly developed reconstitution system, were the subject of the rest of the results chapter. The evaluations of these observations are discussed in the succeeding fourth chapter with respect to the current understanding in the field. The last chapter finally provides a list of the references.

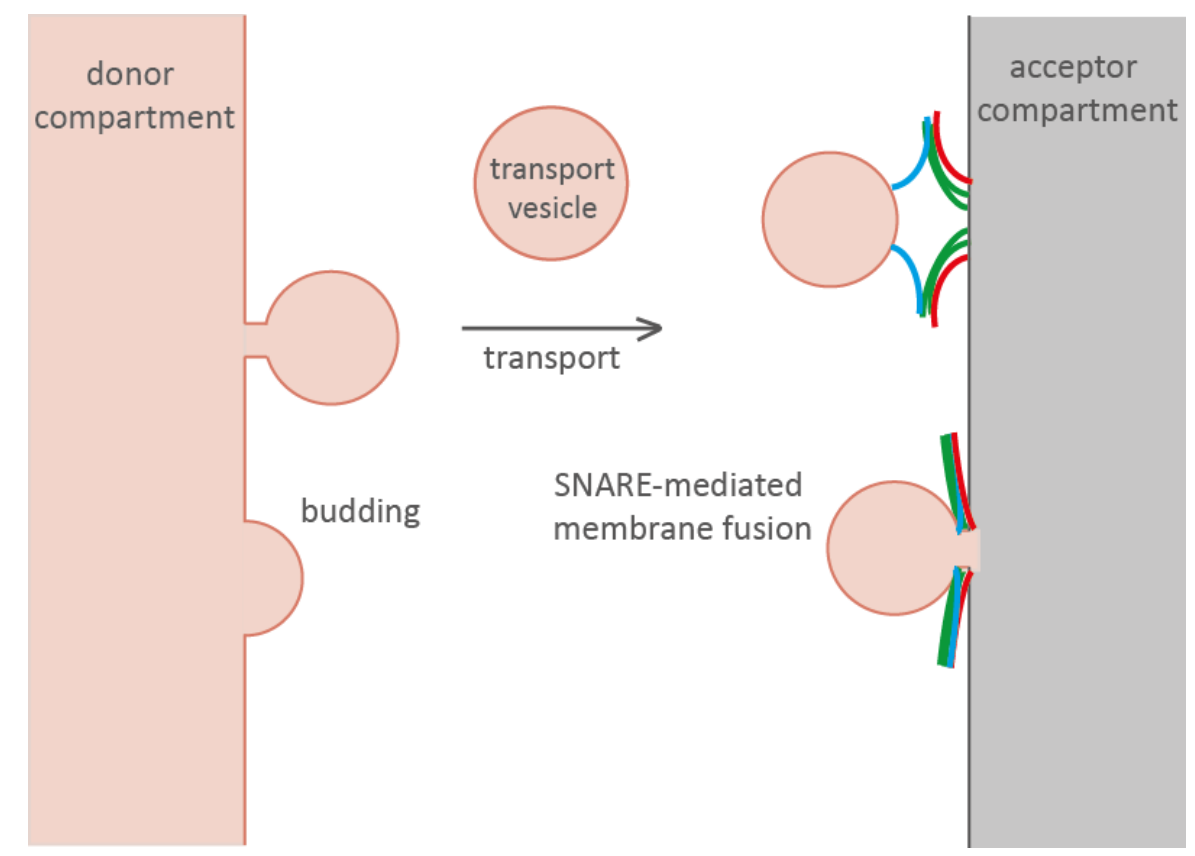

Figure 1.1. A simplified overview of the vesicular transport process. The illustration is modified from [2] with an emphasis on the SNARE-mediated fusion of vesicles to their acceptor membranes. SNARE proteins are depicted in red, green and blue.

\subsection{SNAREs: key members of membrane fusion machinery}

By the late 1970s, it was known that the secretion of newly synthesized proteins was mediated by the fusion of the transport vesicles with their target compartments. Nevertheless, the further understanding of the essential players of this process or their reaction mechanisms was still missing. Following 20 years of research identified the SNARE proteins as the key components of the membrane fusion machinery. 
A pioneering yeast (Saccharomyces cerevisiae) genetic screen was performed by Schekman and co-workers [3]. Each of the temperature sensitive 'sec' mutants they had isolated displayed defects at a particular stage of the secretory pathway. By arranging these mutants into complementation groups, they were able to identify novel genes governing the processes of the secretory pathway, for example the yeast protein Sec18p. This component caught particular attention as its mammalian homolog (Sec18p/NSF) was identified via an independent approach [4, 5]. Rothman and co-workers discovered an N-ethylmaleimide-sensitive factor (NSF) with the help of a cell-free intra-Golgi transport assay [6]. They concluded that N-ethylmaleimide treatment inhibited the in vitro transport reactions between Golgi stacks. NSF, which was shown to be responsible for this outcome, was found both in cytosolic and in membrane bound forms depending on the availability of the ATP nucleotide [4]. Soon it became clear that an adaptor protein was able to bind NSF to the membranes [7]. Three isoforms of this adaptor, soluble NSF attachment protein (SNAP) were purified from bovine brain cytosol and termed $\alpha$-SNAP, $\beta$-SNAP and $\gamma$-SNAP. Later, it was also implicated that there are some integral membrane receptors for NSF/SNAP complexes [8]. Furthermore, $\alpha$-SNAP was identified as a homolog of the yeast protein Sec17p (another "sec" mutant). Hence, the membrane receptors, SNAPs and NSF made up the potent membrane fusion machinery that was presumed to be conserved in eukaryotic fusion reactions [9]. These findings all together raised interest for a search into the membrane receptors that attach to NSF/SNAP complexes.

In 1993, three membrane associated proteins were purified via affinity purification from bovine brain using NSF/SNAP complexes as baits [10]. Later in the same year, these SNAP receptors (SNAREs), synaptobrevin, SNAP-25 and syntaxin were found to assemble in a complex in the absence of NSF and SNAP. The same study also showed that NSF, then identified as an ATPase, disassembled this complex together with SNAPs $[11,12]$.

In parallel, with a more functional approach, targets of clostridial neurotoxins were discovered to be the same proteins as the membrane receptors for NSF/SNAP. By having demonstrated each of these toxins to specifically block neuroexocytosis, these 
Introduction

studies provided direct evidence that the agents driving membrane fusion were the SNARE proteins [13-16].

These two lines of research brought a consensus on the pivotal role of SNAREs in membrane fusion. However, the mechanism of SNARE action and their interactions with NSF were highly debated. The SNARE hypothesis assumed NSF as a fusion factor which acted as an ATPase to trigger the exocytosis [17]. But the evidence from in vitro yeast vacuole fusion reactions clearly showed Sec18p (NSF) as a pre-fusion factor and that further factors were still needed for fusion to occur [18]. The mechanism of SNARE complex assembly was controversial as well. It was known that the three monomers came together and formed a coiled coil. Yet it was unclear whether this was a parallel or antiparallel assembly between the synaptic vesicle and the presynaptic membrane. The organization of the SNARE complex finally became evident as structural data was brought about via Förster resonance energy transfer and electron microscopy techniques $[19,20]$. These data put forward the zippering hypothesis explaining how the membrane distal ends of SNARE monomers "zippered" towards their membrane proximal regions to form complexes that drive membrane fusion. The parallel arrangement of the complex became widely accepted as its crystal structure was resolved in 1998, which set a valuable reference for the subsequent structural and mechanistic studies on SNARE proteins [21].

\subsubsection{SNARE protein structure}

SNARE proteins are members of a highly conserved family of small membrane proteins. They all share a homologous region of 60-70 amino acids, termed the SNARE motif [22]. This region is composed of mostly hydrophobic residues that are arranged in heptad repeats. These properties contribute to the assembly into coiled coils as cognate SNARE monomers come together. A short linker connects the SNARE motif to a C-terminal single transmembrane domain. Most SNAREs also have regulatory domains that are positioned N-terminal to the SNARE motif. However, these different types of individually folded N-terminal domains are not well conserved like the SNARE motifs (for classical reviews see [2, 23]). 
The synaptic SNAREs are good examples for the exceptions of the general domain organization introduced above. Figure 1.2 shows an overview of the domain compositions of these three proteins, as well as their variants which were used in the course of this study. Synaptic vesicle SNARE, synaptobrevin 2 (referred to as syb) belongs to a subset of SNAREs that lack N-terminal domains. It has a very short Nterminal region followed by its SNARE motif, and is attached to the synaptic vesicle membrane via its transmembrane domain. One of the two presynaptic plasma membrane SNAREs, SNAP-25 (referred to as SN25) does not have a transmembrane domain. This unusual SNARE protein has two different SNARE motifs and is anchored to the membrane via palmitoylation at the linker region that connects these two SNARE motifs. The second SNARE on the presynaptic membrane, Syntaxin 1a (referred to as syx) is probably the best fit to the above description. It contains an individually folded regulatory N-terminal domain (Habc domain), a single SNARE motif (H3 motif) and a transmembrane domain.

\subsubsection{Structure of the fully assembled SNARE complex}

Since their discovery, the synaptic SNARE proteins were known to form complexes that are attacked by the ATPase NSF and its adaptor SNAP. Yet, how these assembly and disassembly reactions governed the mechanism of exocytosis became clear only after structural and energetic properties of the SNARE complex were studied.

In the beginning, an interesting observation was made when the recombinant synaptic SNAREs truncated at their transmembrane domains were mixed in solution. The three monomers spontaneously assembled into an SDS-resistant ternary complex [24]. The same study also reported that none of the clostridial neurotoxins were active on this tightly packed complex. More evidence on the tight packing was brought by circular dichroism (CD) and nuclear magnetic resonance (NMR) spectroscopy experiments $[25,26]$. As the SNARE monomers were analyzed, SN25 and syb were found to be largely unstructured and only syx showed $\alpha$-helicity. Surprisingly, the overall thermal stability and $\alpha$-helicity of the ternary complex was substantially higher than its unstructured monomers [25]. 
Introduction
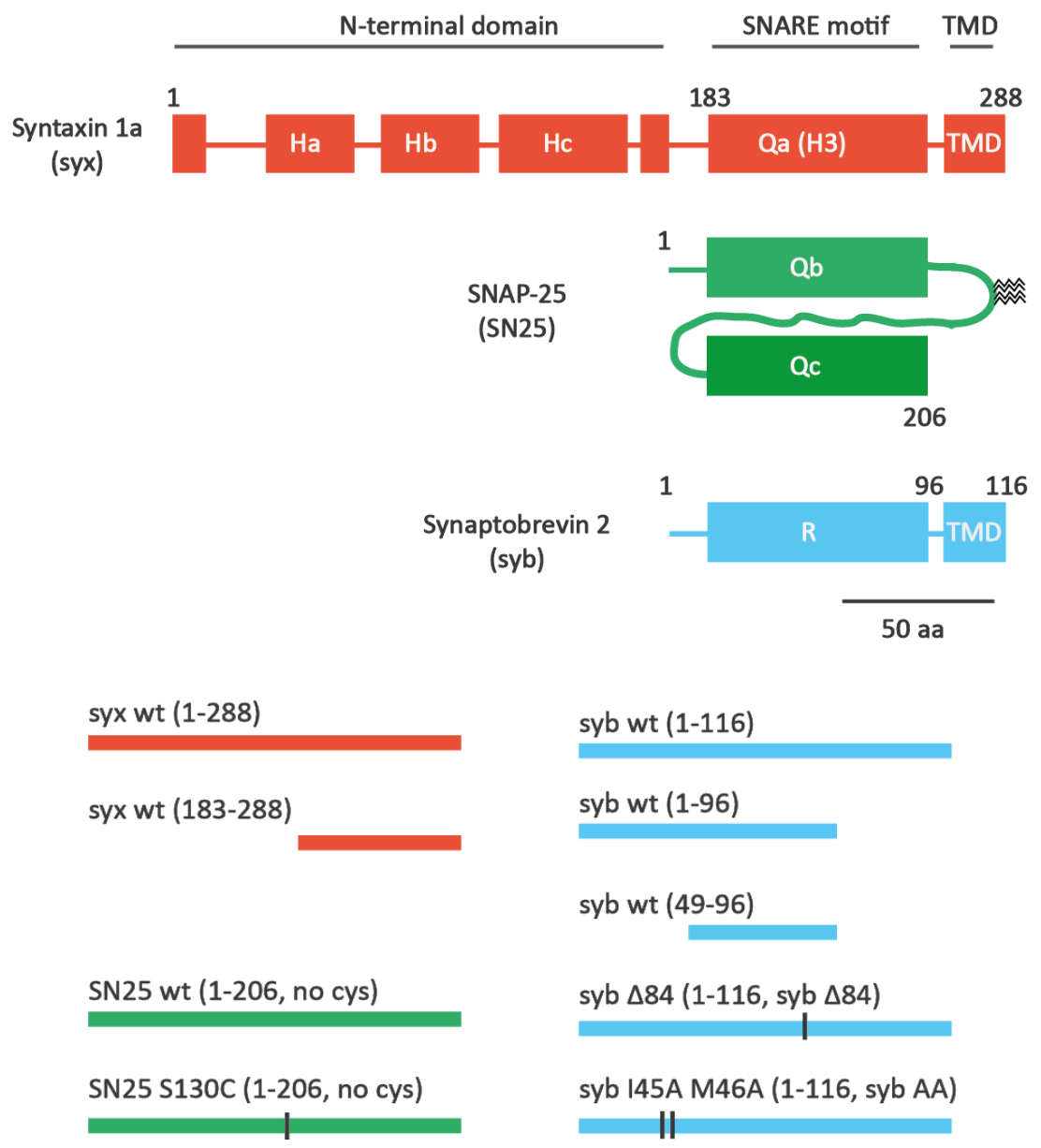

Figure 1.2. Domain compositions of the synaptic SNARE proteins. Top, domain structures of Syntaxin 1a (syx, red), SNAP-25 (SN25, green) and synaptobrevin 2 (syb, blue) are shown. Only syx has an N-terminal domain, the Habc domain. SN25 has two SNARE motifs and lacks a transmembrane domain (TMD). Its linker region with the palmitoylation sites (zig-zag lines) is shown to connect the two SNARE motifs. The SNARE motifs are annotated as $Q a, Q b, Q c$ and $R$ and the principle of this classification is explained in the following section. The residue numbers designate the boundaries of separate domains. $183^{\text {rd }}$ residue of syx marks the beginning of its SNARE motif (Qa or H3) whereas $96^{\text {th }}$ residue of syb corresponds to the end of its cytoplasmic portion. Bottom, schematic representations of the variants of synaptic SNAREs purified for this study. All variants are shown with a line in arbitrary length and mutations are depicted with black dashes. A fulllength syx wild type (wt, 1-288) was prepared via intein purification (for details see 2.2.2.3). The rest of the variants were purified using a hexahistidine tag. Unless stated otherwise all experiments were conducted using an N-terminally truncated syx [27]. A full-length SN25 wt (1-206, all cysteines replaced by serines) as well as its single cysteine mutant (S130C) were prepared. Variants of syb wt in different length and its mutants are shown on the right. For simplicity, single cysteine mutants of syb are not shown. They were all mutated at the $\mathrm{S} 28 \mathrm{C}$ residue, except for syb (49-96), which was mutated at its T79C residue. 
Researchers challenged this highly stable complex further and looked into its essential parts necessary to form a 'core complex'. Recombinant and native ternary complexes were subjected to limited proteolysis by various proteases $[28,29]$. The parts that were removed included the cysteine-rich loop region of SN25 that connects its two SNARE motifs (see figure 1.2). This region was proposed to be unstructured and responsible for anchoring SN25 to the presynaptic membrane via palmitoylation at its cysteine residues [30]. Without the connecting linker SN25 was found in the complex with its two independent fragments corresponding to its two SNARE motifs. The parts that were protected from proteolysis were the N-terminal domain of syx (Habc domain) and a minimal core complex. The transmembrane domains of syx and syb were also protected when they were present at the beginning of the proteolysis. The Habc domain was later shown to form an independently folding three-helix bundle ([31,32] and figure 1.3). And as the core complex components were identified, they revealed the minimal monomers corresponding to four SNARE motifs. Furthermore, these short monomers also assembled spontaneously into SDSresistant minimal complexes and were reversibly disassembled by NSF/ $\alpha$-SNAP [29]. The composition of this minimal complex had been an important guide both to crystallize the SNARE complex and to deduce its function [21].

The crystal structure of the core complex is a 120 A cylinder of the four SNARE motifs of the hetero-trimer. It is a four-helix bundle where all four components are arranged in parallel with their $\mathrm{N}$-terminal regions at the same end of the complex. The distance between the two SNARE motifs of SN25 corresponds to about the length of its linker region. This implies that the linker extends over the entire bundle. Main forces holding this bundle together are the interactions between the side chains of the hydrophobic residues at the inner core of the four helices. These residues are grouped into 16 layers, from the $\mathrm{N}$-terminal $-7^{\text {th }}$ layer to the $\mathrm{C}$-terminal $+8^{\text {th }}$ layer and are numbered with respect to a ' 0 layer'. This is the only hydrophilic layer, among the 15 hydrophobic layers, where positively charged Arg (R) side chain (syb 56) interacts with negatively charged three Gln (Q) side chains (syx 226, SN25 53 and 174). Sequence alignments of the most conserved regions of the SNARE motifs supported 


\section{Introduction}

this main structural arrangement into the hydrophobic layers [33, 34]. These alignments showed a remarkable conservation of the zero layers across the SNARE homologs. Therefore, the SNARE proteins were proposed to be classified corresponding to the amino acid present in the zero layer as Q- and R-SNARE, respectively [34]. Among the Q-SNAREs a sub-classification into Qa-, Qb- and QcSNAREs was established [35]. For example, the synaptic SNAREs syx and the Nterminal and C-terminal SNARE helices of SN25 were named as Qa-, Qb- and QcSNARE, respectively. And syb was named as the R-SNARE of the synaptic complex (see figure 1.2). Like the synaptic SNARE complex, each fusion competent SNARE complex was presumed to be composed of a cognate set of Qa-, Qb-, Qc-SNAREs and an R-SNARE [23].

High degree of conservation of the hydrophobic layer pattern suggested that other SNARE complexes might have a similar parallel four-helix bundle topology. This view became widely accepted as the crystal structure of the distantly-related endosomal SNARE complex was resolved and showed extensive structural similarities to the synaptic complex [36]. Another structural study, which provided further information on the fully assembled SNARE complex, was the crystallization of the helical extension of the synaptic core complex ([37] and figure 1.3). This study investigated a longer core complex including the linker and transmembrane regions of syx and syb that was purified in detergent. It was shown that the longer complex was more resistant to thermal and chemical denaturation. Its crystal structure revealed that both syx and syb form continuous helices not only along their SNARE motifs but also along the linker and the transmembrane regions. The stabilizing side chain interactions that were mapped along the linker region explained why the complex was more resistant against denaturation. This structure corresponds to the state of the fully assembled complex with both transmembrane domains on the same bilayer (defined as the cis conformation) at the end of a round of membrane fusion. 


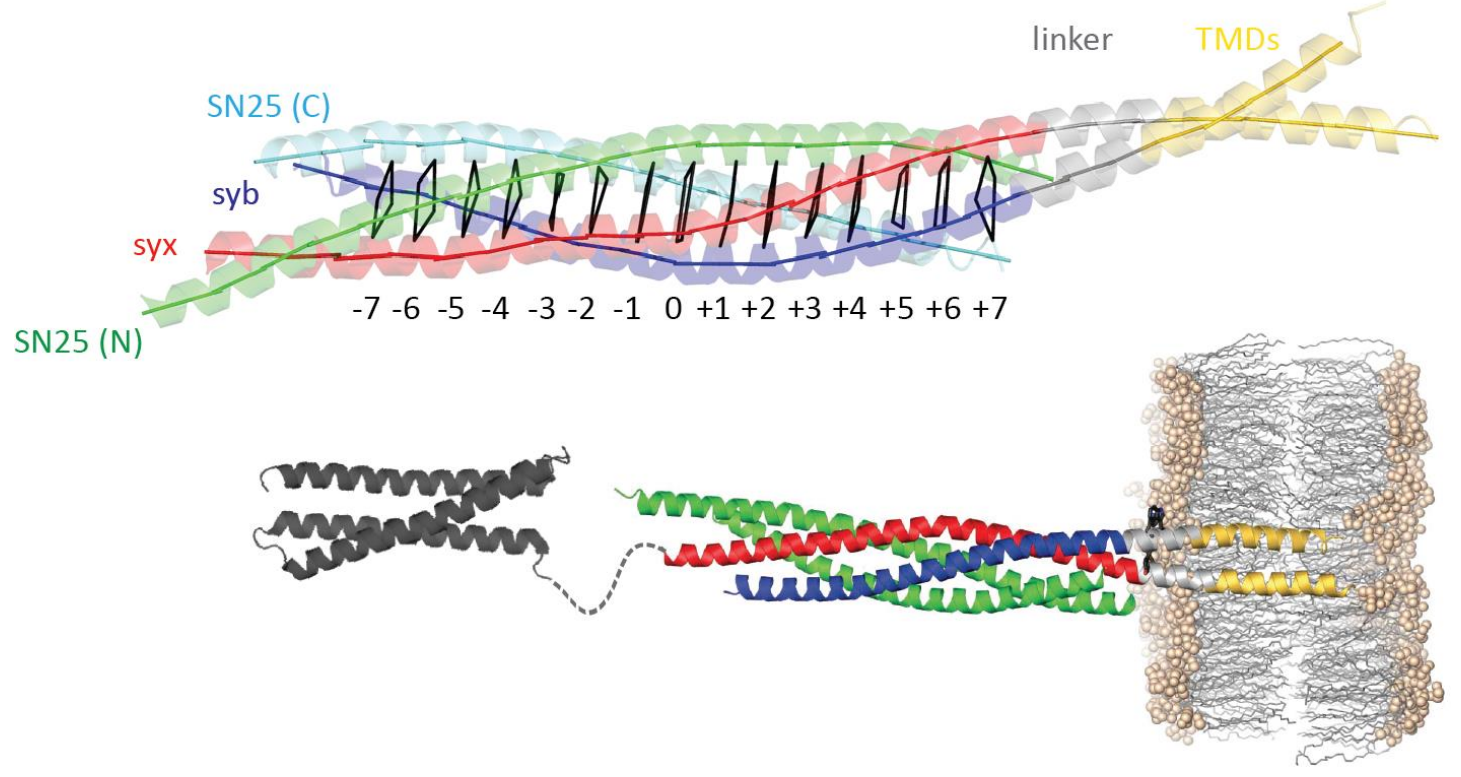

Figure 1.3. Structure of the synaptic complex including the linker and transmembrane domains of syx and syb. Top, the ribbon plot of the N-terminally truncated synaptic complex is shown (kindly provided by Dr. Matias Hernandez and Dr. Gert Weber and modified from [38]). The hydrophobic layers (from -7 to +7 ) are illustrated along the fourhelix bundle. The last $+8^{\text {th }}$ layer is not depicted. Since a part of the SN25 C-terminal helix was absent in the electron density, this layer was not resolved. Bottom, a model of the fully assembled synaptic complex inserted in a PE membrane (modified from [31, 37]). The color code is the same as in the top panel. The crystal structure of the Habc domain of syx is shown in gray and attached to the core complex with dashed lines in arbitrary size. The aromatic residues of the linker region are shown in black.

The crystal structure of the core synaptic complex revealed not only the inner hydrophobic interactions but also the surface interactions [21]. The salt bridges and hydrogen bonds on the surface of the complex demonstrated the amphiphilic nature of the SNARE motif. Shortly after these realizations, various homo- and heterooligomeric complexes of the SNARE monomers were discovered. Among these complexes, homo-oligomers of syx were the first ones to be investigated [30, 39]. At high concentrations, the SNARE motif of syx was found to be assembled into helical bundles, with only some of the $\alpha$-helices organized in parallel. Another complex that received considerable attention was the hetero-oligomeric complex of syx and SN25. In solution syx and SN25 spontaneously assembled in binary complexes with 2:1 stoichiometry; in Qaabc configurations, including N- and C- terminal SNARE motifs of SN25 (Qb and Qc) and two identical SNARE motifs of syx (Qaa) [25]. 
Introduction

Although SNARE monomers were shown to associate in various combinations, not all complexes they formed were parts of the fusion pathway. There were also nonfusogenic SNARE complexes identified with unknown biological significance. An example of such complexes was a Qabab complex of syx and the N-terminal SNARE motif of SN25. The crystal structure of these four SNARE motifs uncovered a parallel four-helix bundle, which failed to react with the SNARE motif of syb [40].

A closer examination of the 2:1 complexes of syx:SN25 was performed via sitedirected spin labeling. These studies reported that the 2:1 complexes also had a fourhelix bundle structure with very similar features to those of the ternary 1:1:1 complexes $[30,41,42]$. Moreover, the second syx was shown to be displaced by the syb monomer [25]. Including these 2:1 complexes, all of the homo- and heterooligomeric SNARE complexes had syx monomer as the common monomer. Also, many salt bridges were identified between syx and SN25 in the synaptic core complex crystal. All together, these observations led to the proposal that binary complexes of syx and SN25 might serve as the precursors in the SNARE complex assembly reactions. The dynamics of such reactions and their precursors are introduced in more detail in the following section.

\subsubsection{SNARE complex dynamics}

It was established that syx and SN25 are presynaptic membrane proteins and syb is an integral membrane protein on synaptic vesicles $[43,44]$. With reference to this topology, it was speculated intuitively that the assembly of these three monomers starts at their N-termini. However, this view was only one of the initially proposed models [45]. As evidence accumulated on the assembly and disassembly cycle of SNAREs, a current understanding has emerged. It is now referred to as the 'zippering hypothesis' based on this N-terminal nucleation proposal.

First, NSF was recognized as an early factor for membrane fusion in an in vitro yeast vacuolar fusion system [18]. Later, like their yeast homologs, synaptic SNARE complexes provided evidence in the same line. In one study, synaptic SNARE monomers were found in the same synaptic vesicle membrane where they were 
assembled into ternary complexes [46]. In the same study, NSF/SNAP were shown to disassemble these synaptic SNARE complexes. Due to the fact that both assembly and disassembly took place on the same synaptic vesicle membrane, it was suggested that NSF disassembly must have been an early step in SNARE cycle driving the fusion of opposing membranes. Additionally, the SNARE complex was shown to be composed of monomers that are arranged in parallel to each other via two independent approaches $[19,20]$. Likewise, in chromaffin cells, an antibody against the $\mathrm{N}$-terminal region of SN25 completely prevented complex formation [47].

These findings together with the structural data (see the previous section) had formed the basis of the zippering hypothesis [23]. This hypothesis asserted that SNARE monomers undergo an assembly and disassembly cycle. After the NSFmediated disassembly, free monomers form fresh complexes in parallel configurations. The best possible way to maintain such a cycle has been explained with the monomers that zipper from their membrane-distal amino termini to membrane-proximal carboxyl termini. During this cycle SNARE complexes exhibit different conformations. The zippering complexes on opposing membranes with transmembrane domains on two different membranes are termed as the trans SNARE complexes. On the other hand, the complexes that form at the end of the fusion process, with all transmembrane domains on the same membrane, are termed as the cis SNARE complexes. The energy release during the conversion process from unfolded monomers to partially folded trans complexes and finally to low-energy cis complexes is thought to fuel the fusion pore opening and the merger of two opposing membranes [48]. Figure 1.4 illustrates the assembly and disassembly cycle of the synaptic SNAREs, the machinery that mediates the neuronal exocytosis process.

In the search for the mechanism of the SNARE complex assembly pathway, a fascinating phenomenon was discovered. Under the conditions that synaptic SNARE complexes were unfolded, no refolding of the monomers occurred [49]. These observations revealed that the assembly and dissociation reactions were energetically separated from each other and that they must follow different pathways. It was put forward that the reason behind this pronounced hysteresis 
phenomenon was a high energy barrier that the assembling monomers must have overcome. With this reasoning, the SNARE complex assembly pathway was suggested to proceed through intermediate states.

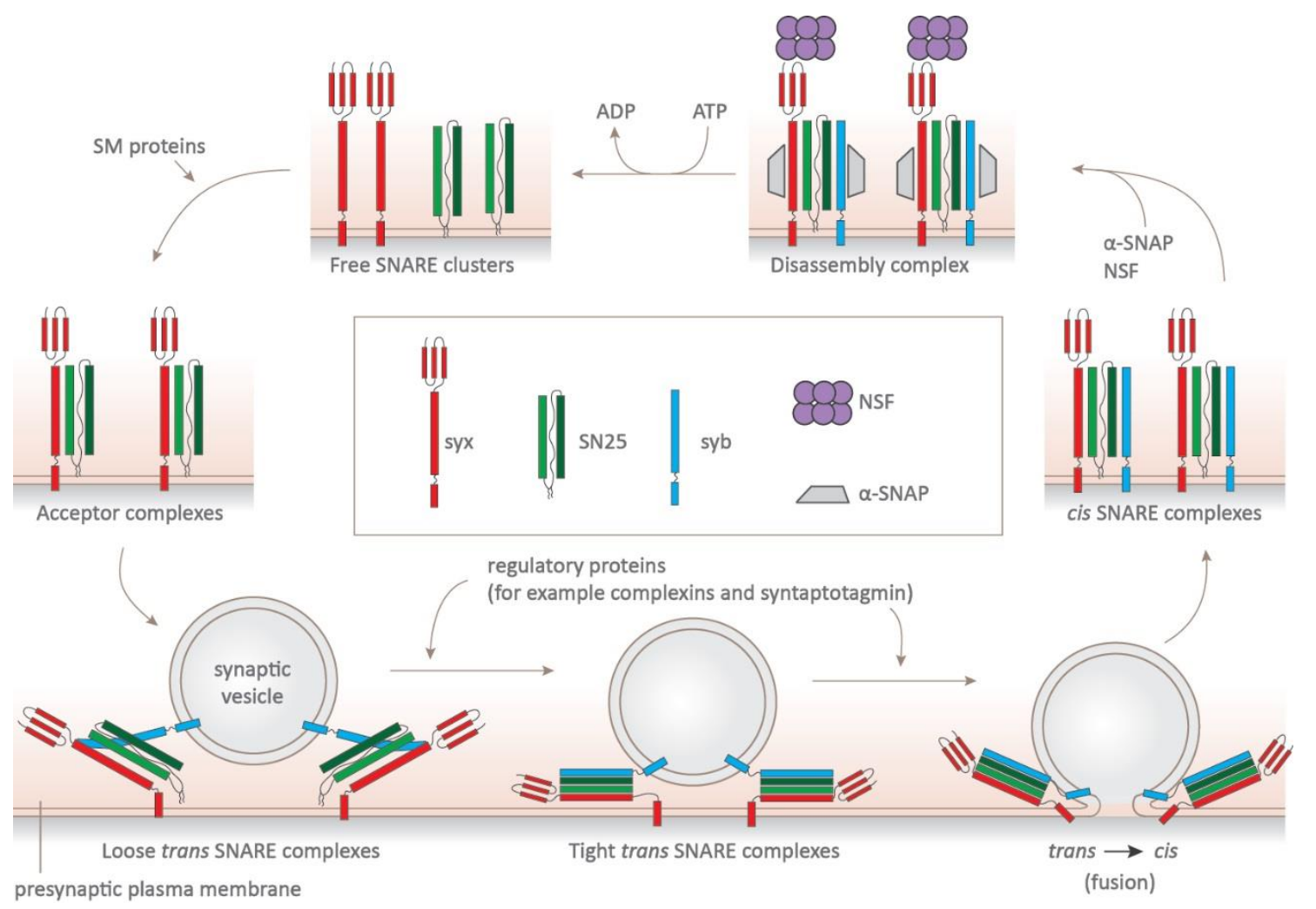

Figure 1.4. Conformational cycle of SNAREs during neuroexocytosis. The illustration was taken from [23] and adapted for the synaptic SNARE cycle.

The evidence of such intermediates first came from recombinant yeast SNARE protein studies. Binary complexes of Sso1p (Qa-SNARE, syx homolog) and Sec9p (Qbc-SNARE, SN25 homolog) exhibited significant folding as unstructured monomers were mixed [50]. These complexes were also shown to have a 1:1 stoichiometry and hence a Qabc configuration [51]. The view that these binary complexes might serve as assembly precursors was supported via independent CD and NMR spectroscopy experiments $[50,52]$. Expectedly, as the pre-assembled binary Qabc complex was mixed with the R-SNARE (Snc1), a rapid formation of the ternary complex was observed. Among the yeast SNARE complex assembly intermediates, one of the most recent and uncommon one was a QabR complex of ER-Golgi trafficking SNAREs [53]. This unexpected combination still provided an acceptor site for the rapid complex assembly. 
In agreement with the zippering hypothesis and the topology of the synaptic SNAREs, spontaneously forming binary complexes of syx and SN25 were suggested to serve as acceptor complexes for the syb monomer [25]. In vitro association of syb with 2:1 complexes of syx:SN25 was possible only when the second syx was displaced by the syb monomer. However, in contrast to the Sso1p:Sec9p (Qabc) complex, the syx:SN25 2:1 (Qaabc) complex did not associate rapidly with the R-SNARE, syb. It was suggested that only the transient syx:SN25 1:1 (Qabc) complex can bind to syb rapidly [54]. Later, this view was strengthened as a biochemically stabilized acceptor complex was generated [55]. This was achieved by leaving the $\mathrm{N}$-terminal nucleation site of the syx:SN25 1:1 complex free and still protecting the syb binding site from the second syx via a C-terminal syb fragment (49-96). Since the resulting complex was Nterminally truncated it was named as the $\Delta \mathrm{N}$ complex. When compared with the syx:SN25 2:1 (Qaabc) complex, the $\Delta \mathrm{N}$ complex was highly reactive in forming a complex with the syb monomer both in solution and on opposing membranes [55].

In order to study the SNARE complex assembly and disassembly dynamics, the $\Delta \mathrm{N}$ complex was used in this study as the acceptor complex for syb. In doing so, the inhibiting role of the second syx in the syx:SN25 2:1 (Qaabc) complexes was avoided. For clarity, during the results chapter the $\Delta \mathrm{N}$ complex was referred to as the acceptor complex.

In most of the studies on the intermediate states of yeast SNAREs and synaptic SNAREs, a general inhibitory role for the N-terminal domains of the Qa-SNAREs had emerged. As recombinant SNARE monomers were mixed in solution, the N-terminal domain of syx was self-associating with the Qa-SNARE motif and preventing syx and its homologs from taking part in complex formation. Therefore the SNARE complex assembly studies were mostly performed with truncated Qa-SNARE domains. It was later revealed that the $\mathrm{N}$-terminal domains of Qa-SNAREs are regulatory regions and soluble factors interact with these regions to stabilize the acceptor complexes. 
Introduction

\subsubsection{Proteins regulating the SNARE complex cycle}

\section{The assembly machinery}

It is now known that prior to the neuroexocytosis of the synaptic vesicles, SNARE complex assembly reaction is highly regulated [56]. In this section, four of the key regulators of this pathway are introduced. Munc18 and Munc13 are the early factors which prepare SNARE assembly intermediates and 'prime' them before the calcium signal. Whereas synaptotagmin and complexin are the late factors which 'trigger' them for calcium-induced membrane fusion.

The two early factors were first identified in a nematode worm (Caenorhabditis elegans) genetic screen of 'uncoordinated' mutant genes as unc-13 and unc-18 [57] in paralyzed worms. It was later discovered that mammalian ortholog proteins Munc13 and Munc18 deletions completely blocked exocytosis [58, 59]. These upstream elements of SNARE complex assembly are believed to regulate the stability of the acceptor complexes and perhaps even the trans SNARE complexes [56].

Munc18 is a member of a conserved family of cytosolic proteins of about 600 residues, named as Sec1p/Munc18-like (SM) proteins. SM proteins share a characteristic archshaped structure with a central cavity. It is currently believed that SM proteins interact mainly with the N-terminal domains of their cognate syx, thereby stabilize the monomer as it takes part in acceptor complex and/or SNARE complex formation. Via this mechanism, SM proteins were suggested to be brought to the fusion site and associate with the helical bundle of the assembling SNARE motifs (a function which is proposed for their central cavity) [56, 60, 61]. For example, the yeast SM protein Vps33p has been shown to facilitate yeast SNARE complex assembly in vitro [62]. However, the SM protein of the neuronal exocytosis, Munc18, initially did not fit into this model. Even though it was identified as an essential component of the fusion machinery together with the SNARE proteins, its mode of action remained unclear [63]. Deletion of Munc18 in neurons blocked the neurotransmitter secretion completely, which demonstrated Munc18 as a vital element of the fusion machinery. In parallel, the N-terminal domain of syx was shown to self-associate with its SNARE 
motif and adapt a "closed" conformation. Munc18 was found to bind to this conformation and lock syx in its monomeric form $[64,65]$. It was difficult to reconcile these two lines of research. If Munc18 was an indispensable element, and a lock for syx monomer, how come syx would take part in SNARE complex formation in the presence of Munc18? A general picture of Munc18 function has emerged after two binding sites on Munc18 with different affinities were studied [66-68]. It is now believed that Munc18 keeps syx closed and perhaps inactive, but upon the calcium signal, it also supports syx in complex formation. These events might occur via a possible interaction of Munc18 with the trans SNARE complexes, as in vitro reconstitution of neuroexocytosis studies reported Munc18 to mediate SNARE complex nucleation $[69,70]$.

The second essential factor for exocytosis, Munc13, has a distinctive domain structure with a phorbol-ester-binding $\mathrm{C} 1$ domain, two calcium-binding $\mathrm{C} 2$ domains and a socalled MUN domain [71]. As it had been difficult to express the full-length recombinant protein, a minimal MUN domain has been studied. This fragment has been shown to partly rescue the neurotransmitter release in neurons lacking Munc13 [72]. As its crystal structure was resolved, the MUN domain has been found to be remarkably similar to the members of the CATCHR protein family (Complex Associated with Tethering Containing Helical Rods) [73]. CATCHR proteins are modular proteins which are identified as rod-like domains of helical bundles. Via these tertiary structures they are proposed to accommodate SNARE complexes and/or the regulatory proteins associated with the complex assembly. For instance, a CATCHR protein, Dsl1p of yeast has been reported to stabilize the Qabc acceptor complex of endoplasmic reticulum SNAREs. A similar function for Munc13 has been proposed. Fluorescence and NMR spectroscopic experiments put forward that Munc13 might be responsible for the transition between the closed syx/Munc18 complex to fully assembled SNARE complex [74, 75]. However, at which step of this sequence Munc18 is released from the SNARE complexes is still not clear.

In addition to the upstream regulation of SNAREs exerted by Munc13 and Munc18, neurons and neuroendocrine cells require a tight regulatory mechanism. Therefore, 


\section{Introduction}

the fast coupling of excitation to the neurotransmitter release can be achieved in milliseconds. Synaptotagmin and complexin are the components of the downstream regulation of SNAREs at the presynaptic nerve terminal [56]. They ensure the fast synchronous release when an action potential induces the calcium trigger. The SNARE monomers "primed" by Munc13 and Munc18 are "triggered" by synaptotagmin and complexin to drive synaptic vesicle exocytosis.

Neuronal synaptotagmin resides on the synaptic vesicles [76]. It is anchored to the membrane via its $\mathrm{N}$-terminal single transmembrane domain. Its cytosolic domain is made up of two calcium-binding C2 domains. Both C2 domains are connected to each other and to the transmembrane domain with flexible linkers. It functions as a calcium sensor at the presynaptic terminal. Upon the arrival of the calcium signal, it binds to both the membrane and the assembling SNARE complex [77]. It has been reported to associate with the presynaptic membrane or to crosslink the two opposing membranes [78-80].

Complexin on the other hand, belongs to a small family of soluble and charged proteins [81, 82]. It was initially found to be co-localized with or bound to the SNARE complex [83]. As its SNARE complex-bound form has been crystallized, an interaction between its central $\alpha$-helix and a surface groove of the SNARE complex was discovered [84]. In addition to the central $\alpha$-helix, an accessory $\alpha$-helix was also reported to associate with the assembling SNARE complexes. This helix is believed to interrupt the SNAREs from further zippering [85]. Evidence from different lines of research proposed both positive and negative roles for complexin. Even though it has been extensively discussed, complexin is now believed to attach itself to the trans SNARE complexes via its central helix and the inhibitory accessory helix is displaced upon the calcium signal [86-88].

It has been highly debated how synaptotagmin and complexin work, perhaps synergistically, on the zippering SNARE complexes. Two scenarios have been proposed with respect to the half-life of the partially assembled SNARE complexes [56]. Both cases explain the stage where acceptor complexes or freshly nucleating 
complexes are stabilized by the upstream elements, Munc13 and Munc18. Upon calcium triggering they proceed via two different mechanisms. According to the more widely accepted first scenario, complexin clamps the late zippered complex via its accessory helix and synaptotagmin removes this clamp when bound to calcium. The second scenario, however, claims that syb binding to the acceptor complexes occurs only after the calcium signal, as synaptotagmin brings the opposing membranes closer. Once the SNARE complex nucleation has started, complexin helps to stabilize these early zippering complexes (see figure 1.5 and figure 3 in [56]).

Either path eventually leads to the conversion of the trans SNARE complexes to the cis SNARE complexes. This establishes the fusion pore opening and the neurotransmitter release. Once the SNARE monomers are found in low-energy complexes, they do not dissociate spontaneously [49]. Hence, they must be disassembled to become re-energized for the following rounds of secretion. This is achieved by the ATPase NSF and its co-factor SNAP by using the hydrolysis of ATP as an energy source.

\section{The disassembly machinery}

NSF belongs to the family of the ATPases associated with various cellular activities $(A A A+)$. The hallmark of the members of this family is that they couple ATP hydrolysis to large conformational changes or re-modelling of protein assemblies of various processes in the cell [89]. NSF has two of the characteristic AAA ATPase domains, the D1 and D2 that mediate SNARE complex disassembly [11]. The D1 domain is responsible for the main disassembly reaction, whereas the D2 domain maintains an ATP-dependent oligomerization of the NSF subunits [90, 91]. In addition to the AAA domains, NSF contains a very flexible $\mathrm{N}$ - terminal $\mathrm{N}$ domain which is required for the binding to the co-factor SNAP and to the SNARE complex. N, D1 and D2 domains make up one subunit of an NSF hexamer [92]. A wealth of structural data has been gathered on the domains of the NSF subunits and the hexamers they organize into [20, 92-96]. 


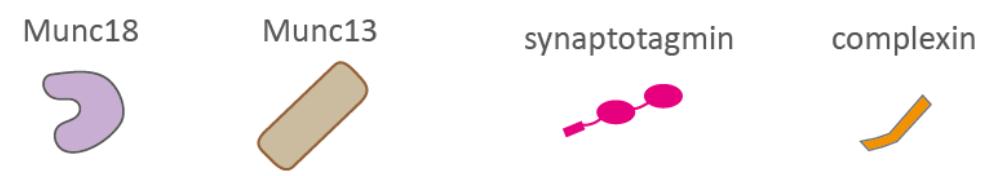

\section{Model I (with long-lived partial complex)}

priming I

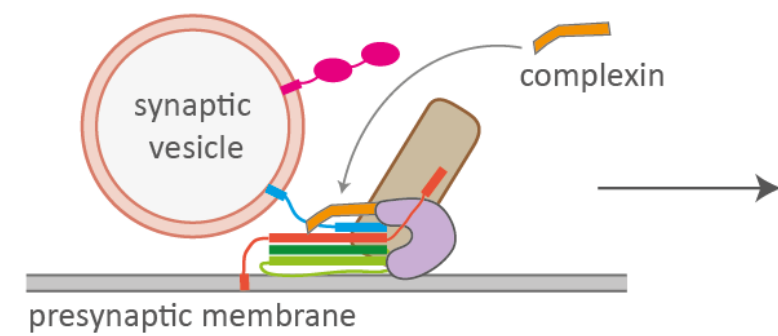

presynaptic membrane triggering I

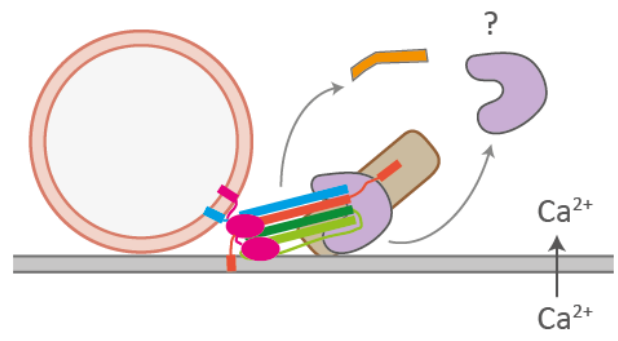

Model II (with short-lived partial complex)

priming II

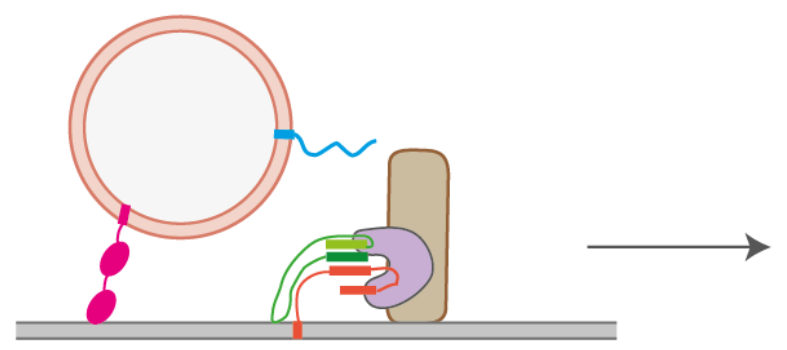

triggering II

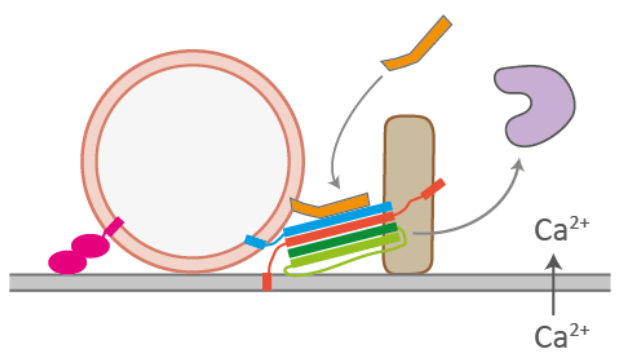

Figure 1.5. Two models describing the transition between priming and triggering steps before synaptic vesicle exocytosis. The illustration was adapted from [56].

NSF alone is not able to bind to the SNARE complexes. It requires a co-factor, SNAP. There are three mammalian SNAP isoforms, identified as $\alpha$-, $\beta$-, and $\gamma$-SNAPs [7]. $\alpha$-, and $\gamma$-SNAPs are ubiquitously expressed, whereas $\beta$-SNAP is a brain-specific isoform [97]. The crystal structure of the Sec17p (yeast $\alpha$-SNAP homolog) revealed its $14 \alpha$ helices assembled in an asymmetrical structure [98]. The availability of its structure and the fact that it is the yeast homolog made the recombinant $\alpha$-SNAP a popular probe for the following research. Recently, an N-terminal loop region was discovered that serves as a conserved membrane attachment site [99]. Interestingly, this region increased $\alpha$-SNAP's affinity (to about 20-fold) to the membrane-bound SNARE complexes. These findings highlighted the importance of investigating the protein- 
Introduction

protein interactions using in vitro reconstitution systems that incorporate a membrane environment.

Before the discovery of the SNARE proteins, NSF and SNAP were identified in a 'multisubunit particle' with the SNARE complex in a detergent solubilized system. This essential particle of membrane fusion was referred to as the ' $20 \mathrm{~S}$ particle' with regard to its sedimentation coefficient [8]. Later studies, mainly via electron microscopy, focused on how NSF, $\alpha$-SNAP and the SNARE complex are organized into this large assembly $[20,100,101]$. According to the widely-accepted stoichiometry, three SNAP molecules mediate the binding of one NSF hexamer to one SNARE complex $[101,102]$. A recent $3 \mathrm{D}$ reconstruction of the $20 \mathrm{~S}$ particle has been put forward that aimed to combine all structural data available [103].

Like the stoichiometry of the $20 \mathrm{~S}$ particles, how the energy is transformed through this structure to disassemble the SNARE complexes has also been debated. Two contradicting models have recently attracted attention. One model proposes a stepwise destabilization of the complex upon multiple rounds of ATP hydrolyses $[104,105]$. On the contrary, the second model assumes that NSF destabilizes the entire complex globally possibly in one round of ATP hydrolysis [106].

Even though the stoichiometry and the mechanism of disassembly remain elusive, it is clear that both native and recombinant cis complexes are recognized by SNAPs and disassembled by NSF $[10,12,46,107]$.

\subsection{Partially assembled SNARE complexes}

From the description above, it is evident that several aspects of the SNARE complex assembly/disassembly cycle are not entirely clear and still discussed controversially. According to the zippering hypothesis, the key step in SNARE assembly involves the transient formation of trans complexes. The nucleation initiates at the membranedistal amino termini of the SNARE motifs and progresses towards the membraneproximal carboxyl termini. One of the reasons that the SNARE dynamics are still open to discussion since years has been the difficulty to indisputably prove the existence 
Introduction

of the trans complexes. This was partly due to their transient nature. It has been challenging to isolate these structures without converting the trans conformation into cis conformation, especially via detergent solubilization.

\subsubsection{Experiments in intact cells}

Although indirect, the most valuable evidence for the existence of the trans complexes were obtained with the studies of regulated exocytosis in neurons and neuroendocrine cells [108-110]. In crayfish neuromuscular junctions, two different clostridial neurotoxins, tetanus neurotoxin (TeNT) and botulinum neurotoxin B (BoNT/B) were injected into the axon of the excitatory synapses [109]. These toxins digest the unfolded synaptobrevin monomer (syb) at the same cleavage site when it is not involved in a SNARE complex. TeNT recognizes an N-terminal site of the monomer, whereas BoNT/B recognizes a C-terminal region. Interestingly, only BoNT/B processed the syb monomer both before and after nerve stimulation. TeNT, on the other hand, cleaved the monomer only after the nerve activity. These findings were best explained by the trans SNARE complexes that had assembled prior to nerve stimulation. In this state the complex is N-terminally zippered, hence TeNT could not recognize the syb monomer. However, the C-terminal region of the complex had not yet folded and this region of the syb monomer was still susceptible to BoNT/B cleavage [109]. Only after stimulation, when NSF had recycled SNARE complexes into unfolded monomers, then TeNT cleaved the syb monomer and blocked neurotransmission. Likewise, a set of functional data obtained in a cracked PC 12 cell system suggested that SNAREs assemble in partial complexes before the calcium signal arrives and triggers nerve activity [110].

In 1998, Neher and co-workers introduced an inventive electrophysiological method that distinguishes kinetically distinct pools of secretory vesicles in chromaffin cells [108]. Each pool of vesicles represents sequential steps of SNARE-mediated fusion. In this method, upon ultraviolet radiation previously caged calcium is released, thus an exocytotic response is generated in heterogonous release probabilities [111]. Measurements of the vesicle fusion (via membrane capacitance) and of the hormone 
release (via carbon fiber amperometry) revealed two kinetically distinct responses. First, a rapid burst of exocytosis was observed, which corresponded to the readily releasable pool (RRP) of vesicles. Second, a sustained response at high calcium levels was caused by the fusion of vesicles from the slowly releasable pool (SRP). Prior to the calcium signal, RRP vesicles were suggested to be already primed with partially assembled SNARE complexes. Hence, rapid burst analyses provided information on the release kinetics, that is, the late regulatory steps of triggering, which may involve calcium responsive elements like synaptotagmin and complexin. On the other hand, SRP vesicles were assumed to have SNARE monomers that were not yet assembled into complexes. Thus, sustained response analyses revealed the early regulatory steps of priming that involved Munc13 and Munc18.

Regulated exocytosis was investigated in chromaffin cells in various ways, by using clostridial neurotoxins, anti-SNARE antibodies or toxins against the disassembly machinery. These investigations not only provided evidence on SNARE complexes assembled in trans, but also proposed a dynamic equilibrium between their loose and tight conformations $[47,108,112]$. They supported a model which was suggested by Hanson and co-workers [20], where NSF and $\alpha$-SNAP are early factors disassembling SNARE complexes and allowing them to reassemble in productive fusion-competent complexes (productive re-assembly model).

In 2005, a double knock-out (dKO) mouse for both synaptobrevin and cellubrevin was generated [113]. The chromaffin cells isolated from these animals provided an extremely useful medium to express variants of synaptic SNARE monomers in null background [114-116]. The motivation behind these studies was to interfere with the stability of the zippering trans complexes by mutating the hydrophobic layer residues in the SNARE motifs. Electrophysiological and biochemical experiments were performed with the layer mutants of SN25 and syb expressed in dKO cells. They revealed two functional regions in the SNARE bundle that can fold and unfold independently $[114,115]$. These results were interpreted such that the $\mathrm{N}$-terminal region of the zippering bundle might contain a "switch" that is important for further zippering upon the transition from priming to triggering. This view was supported by 
Introduction

additional computational, electrophysiological and biochemical experiments. Remarkably, such a switch was identified by introducing double alanine substitutions around the $-3^{\text {rd }}$ layer of synaptobrevin (syb I45A M46A, syb AA) [116]. The concept of switch sequences in the SNARE motif which promote further zippering has caught attention recently as it was also put forward by independent experimental approaches using magnetic and optical tweezers, as well as fluorescence spectroscopy [117-119].

\subsubsection{Experiments on cell-free native membranes}

In addition to the investigations done in intact cells, the existence of the trans SNARE complexes was implicated by other approaches. Among these approaches were the cell-free assays performed with isolated organelles [120]. An intensive series of studies on the fusion mechanisms of isolated yeast vacuoles was conducted by Wickner and co-workers [121]. They developed cell-free yeast vacuole fusion assays. As the isolated vacuoles fused with each other, these assays measured the mixing of vacuolar contents colorimetrically $[122,123]$ or the mixing of vacuolar lipids fluorescently [124]. The content mixing experiments involved two different yeast strains expressing the inactive forms of the colorimetric reporters. One content mixing assay monitored the maturation of the yeast alkaline phosphatase Pho8p [122], whereas another one monitored the complete folding of a tripartite enzyme $\beta$ lactamase [123]. Pho8p and $\beta$-lactamase became active only when the isolated vacuoles fused and their otherwise inactive contents mixed. The fusion thereafter was assayed as the substrates of the active enzymes were processed.

Using these assays, a working model of yeast vacuolar fusion was constructed. According to this model, Sec17p ( $\alpha$-SNAP homolog) and Sec18p (NSF homolog) are the early factors in SNARE-mediated fusion reaction [18]. They are proposed to disassemble the cis SNARE complexes that are found on the isolated vacuoles [125]. This step generates re-energized SNARE monomers ready to assemble with the cognate monomers on the opposing vacuolar membrane in trans conformation [126, 127]. Finally, the HOPS complex accommodates the conversion of the trans complexes 
into cis complexes, which results in vacuolar fusion [128, 129]. Even though this model was built mostly by assaying membrane fusion, recently trans SNARE complexes were also assayed in detergent extracts directly, using epitope tags [127]. With this method, a SNARE complex in a partially zippered conformation was demonstrated [130]. Spontaneous full zippering of the cognate SNAREs on opposing membranes was prevented by using excess amounts of a C-terminally truncated soluble Qc-SNARE monomers. This truncated monomer stopped vacuolar fusion, therefore the trans complexes were claimed to be trapped in partially zippered states [130].

\subsubsection{Experiments in biochemical reconstitution systems}

Since 1998, one of the major goals of the SNARE-mediated fusion studies has been to recapitulate the SNARE function in vitro. Several minimalistic reconstitution systems, which involved biochemically defined components on artificial membranes, were developed to monitor membrane fusion $[27,131,132]$. The experiments conducted in these systems contributed immensely to the prevalent understanding on the mechanisms of SNARE-mediated membrane fusion. This section focuses on the ones that proposed the presence of a metastable trans complex intermediate in their fusion pathways $[126,133]$.

The model for yeast vacuolar fusion which was based on the studies with isolated vacuoles (see above) was further supported with an in vitro system containing liposomes reconstituted with yeast SNAREs [132]. The researchers reproduced lysisfree liposome fusion reactions and drove conclusions on the assembly of trans SNARE complexes [134-136]. A very interesting message these experiments had delivered was a synergy between phosphoinositides and the regulatory proteins acting on SNARE complex assembly intermediates. They propose that all regulatory factors act synergistically on yeast SNAREs to let them assemble into highly fusogenic trans SNARE complexes starting at their membrane-distal amino terminals $[137,138]$.

Like their yeast homologs, synaptic SNAREs were also reconstituted on artificial membranes and have served as popular tools to study regulated neuroexocytosis [27, 
Introduction

131]. A widely utilized liposome fusion assay provided insights into partially zippered complexes [131]. This classical assay was based on reconstituting cognate synaptic SNAREs on two different populations of liposomes, one with fluorescently labeled lipids and another with unlabeled lipids. Upon mixing the two kinds, liposomes fuse and labeled/unlabeled lipids mix. By measuring the fluorescence dequenching upon lipid mixing, scientists were able to observe SNARE-mediated membrane fusion.

Early investigations of synaptic SNARE liposomes were measured with this approach. Synaptobrevin liposomes were pre-incubated with co-purified syx:SN25 complex liposomes at low temperatures (overnight incubations at $4^{\circ} \mathrm{C}$ ). It was suggested that under low temperatures liposomes did not fuse and trans SNARE complexes were trapped in between docked liposomes [133]. Later in the same system, the liposomes were mixed with Munc18 during the pre-incubation at low temperatures. At the end of these incubations the temperature was elevated and lipid mixing upon liposome fusion was measured. Since the presence of Munc18 increased the fusion rate, it was concluded that Munc18 was responsible for stabilizing trans SNARE complexes and making them more fusogenic $[69,70]$.

A very similar lipid mixing method was used in a different reconstitution system of synaptic SNARE liposomes. This system utilized a stabilized acceptor complex (the $\Delta \mathrm{N}$ complex) of syntaxin and SNAP-25 for synaptobrevin binding [55]. In this way, the inhibitory effect of the second syntaxin molecule in the synaptobrevin binding site was prevented. Hence, the measured lipid mixing in this system reflected the liposomes fusion upon trans SNARE assembly more directly. Proceeding studies of this set-up included synaptobrevin variants that were mutated at their critical hydrophobic layer residues. These mutations rendered the hydrophobic interactions of the SNARE motif weaker and lowered the overall SNARE complex stability. It was presumed that zippering was retarded with long-lived trans SNARE complexes between the liposomes that were about to fuse because these mutants showed negative effects on liposome fusion rates [116, 139]. One of these synaptobrevin mutants was a deletion mutant which lacked the $84^{\text {th }}$ residue at the most $\mathrm{C}$-terminal layer of its SNARE motif (the $+8^{\text {th }}$ layer), further referred to as syb $\Delta 84$. Large 
liposomes (diameter, $100 \mathrm{~nm}$ ) that were reconstituted with syb $\Delta 84$ failed to fuse with other large liposomes containing acceptor complexes. Instead, these liposomes were tightly docked to each other, suggesting that they contain arrested trans complexes at the membrane contact site [38].

Other in vitro systems used different approaches to reconstitute trans complexes. Some aimed at generating partially assembled complexes using liposomes and planar membranes, thus creating a topology more similar to the fusion between synaptic vesicles and the relatively flat presynaptic plasma membrane. In these systems, RSNARE liposomes were incubated on planar surfaces with immobilized Q-SNAREs. For instance, a bulk assay was developed to search for trans SNARE complexes assembled between Rhodamine loaded synaptobrevin liposomes and a planar surface with immobilized syntaxin [140]. When soluble SNAP-25 monomers were added to this system, liposome docking on the planar surface was mediated by three monomers assembling in trans. Liposome docking was assayed by the fluorescence of Rhodamine. The resolution of this approach was improved by introducing methods that allow for monitoring single vesicles [141]. Via total internal reflection microscopy, single synaptobrevin liposomes were monitored as they fused with the planar supported bilayers that were reconstituted with the stabilized acceptor complexes.

The current working model, which is based on such biochemical systems, explains synaptic SNARE-mediated membrane fusion with a synergistic mechanism similar to the one proposed by Wickner and co-workers $[75,132]$. However, this model does not address the nature of the trans SNARE complexes. Like in most of the reconstitution systems, in this system trans SNARE assembly and fusion occur spontaneously. The conclusions regarding the partially assembled complexes are mostly indirect, being derived from data on membrane fusion using lipid probes. It remains unclear how regulatory proteins interact with these complexes. 
Introduction

\subsubsection{Trans SNARE complex dynamics at the synapse}

Up to now, many investigations have focused on the interactions between regulatory factors and the synaptic trans SNARE complexes with the approaches summarized above. Nevertheless, the conclusions drawn from the data from different approaches contradicted each other. For example, there are two opposing views on how NSF interacts with partially assembled complexes. According to one view, as trans SNARE complexes assemble they become resistant to NSF-driven disassembly and are committed to membrane fusion $[133,142]$. Another line of research states that these partially assembled complexes are indeed disassembled by NSF [126]. Intriguingly, the same study also states that this disassembly has no inhibitory effect on the proceeding membrane fusion. Furthermore, the co-factor $\alpha$-SNAP and its yeast homolog have been reported to have both activating [130] and inhibiting [143] roles on the zippering complexes of native yeast vacuoles and synaptic vesicles, respectively. All together with these findings it is difficult to presume the state of trans SNARE complexes in the presence of an active disassembly machinery at the presynaptic site.

Another controversially discussed mode of regulation of the trans complexes is exerted by synaptotagmin and complexin. Their interplay upon the calcium influx is still unclear. According to the models that were introduced above (see figure 1.5 and [56]) two alternative mechanisms were proposed. Complexin might be bound to the long-lived trans complexes and get displaced by synaptotagmin upon the arrival of the calcium signal (model I). Alternatively, it might interact with the zippering complexes that nucleate only after the calcium influx. This interaction might stabilize the short-lived trans complexes in a synaptotagmin-independent manner (model II).

Clearly, the mechanisms proposed by these models are very different from each other. Moreover, how NSF and its co-factor SNAP take part in this sequence of events remains unclear. If there is a long-lived trans complex intermediate: to which extent is it zippered? Does it bear a binding site for SNAP? Is it disassembled by NSF, or do factors like Munc13 or Munc18 protect such fusogenic complexes from disassembly? 
Other probable roles for NSF and SNAP might involve disassembly of non-fusogenic complexes (i.e. trapped complexes) and thus continuously regenerate fresh SNARE monomers [40].

The key for elucidating the transition from the primed SNAREs to the triggered SNAREs relies on understanding the dynamics of trans complexes. These metastable complexes are vital to investigate since they are the subjects of the main regulations leading to the neurotransmitter release. The problem with the aforementioned approaches is that they mostly measured the dynamics of the partial complexes indirectly. In most biochemical reconstitution systems, lipid or content mixing is measured as liposomes fuse. Reaching conclusions on protein-protein interactions based on the read-outs of membrane dynamics usually led to inconsistencies. For instance, in a study using an in vitro approach it was stated that the disassembly of the trans complexes did not interfere with membrane fusion [126]. This is essentially conflicting with the zippering hypothesis, as no disassembled trans complex can continue zippering and be converted to a cis complex to drive fusion.

In order to understand their function better, trans complexes must be monitored directly. So far only a few direct trans complex probes were introduced $[38,127,130]$. It is still essential to correlate the read-outs from both trans complex probes and membrane dynamics probes. With the molecular tools that are available, it is possible to capture trans complexes biochemically. Regulatory factors like complexin or Munc18 might be tested as to whether they are able to rescue such artificially trapped trans complexes and let them zipper further. Monitoring both trans complex dynamics and membrane fusion during such rescue experiments would point to the role of the regulation mechanisms on the trans complexes leading to fusion. 
Introduction

\subsection{Aims of this study}

This study aimed at developing a biochemically well-defined reconstitution system in which the dynamics of the partially assembled synaptic trans SNARE complexes could be directly monitored. These very transient, metastable species were planned to be captured between liposomes that are docking but failing to fuse. When constructing this novel reconstitution system, the advances from the previous systems were taken into consideration.

Recently, synaptobrevin variants have been reported with mutations in their critical hydrophobic residues of their SNARE motifs (see sections 1.2.1 and 1.2.3). These mutations have negative effects on the overall reactivity of the zippering SNARE complexes between liposomes and consequently lower the fusion rates of these liposomes [116, 139]. Among them, the so-called "docking mutant", the deletion mutant, syb $\Delta 84$ was of particular interest for this study [139]. On large liposomes, it binds to the acceptor complex, just like a wild type synaptobrevin monomer. This binding mediates the docking of syb $\Delta 84$ liposomes to acceptor complex liposomes. However the docked liposomes do not proceed to fuse. Because of the energy minimum which is brought about by the low curvature stress of large liposomes, syb $\Delta 84$ zippering stalls around the $+8^{\text {th }}$ layer [38]. At this time point, the large liposomes become arrested at the docked state with trans SNARE complexes trapped between the two opposing liposome membranes. These compelling observations proved that the deletion mutant and the large liposomes would provide a set of valuable tools for this study.

The second component of this tool box was a double substitution mutant of synaptobrevin, syb AA [116]. This mutant has two alanine substitutions at the $-3^{\text {rd }}$ layer of the synaptobrevin SNARE motif. Previously, the region between $-4^{\text {th }}$ to $-2^{\text {nd }}$ layers was proposed to exhibit the characteristics of a coiled coil which serves as a molecular switch that allows further zippering. Without this switch, the syb AA mutant exhibits a profoundly lowered initial fusion rate of the small liposomes [116]. These findings offered a candidate for a second synaptobrevin mutant that traps 
partially assembled complexes under lower curvature stress. Therefore, one of the goals of this study was to reconstitute the syb AA mutant on large liposomes and to test whether it exhibited a "docking phenotype" similar to the syb $\Delta 84$ mutant. This way, a second type of trans complex might be generated that is partially zippered and arrested at the more $\mathrm{N}$-terminal -3 layer which is more distant from the membrane anchor domains.

Initially, three different reconstitution systems were developed using the syb $\Delta 84$ mutant on large liposomes. Among these systems, the one that suited best to the aim of the study was chosen. The second part of the project was then focused on characterizing the trans SNARE complexes that involved the syb $\Delta 84$ mutant. It was planned to monitor how these trans complexes interacted with the purified factors regulating neuronal exocytosis. Once the reconstitution system with syb $\Delta 84$ mutant was established, the very same approach was used to construct a sister system with the syb AA mutant. The objective of having two systems for both mutants was to study the effect of factors like NSF on partially assembled complexes with different conformations. Finally, this study intended to elucidate the dynamics of the liposomes reconstituted with trans complexes. The ultimate goal in doing this was to provide the necessary tools to monitor the trans SNAREs and the opposing membranes in the same medium. Therefore, a correlated understanding would be reached on how trans SNARE complex regulations are reflected on liposome docking or fusion events. 
Introduction 


\section{Materials and Methods}

\subsection{Materials}

\subsubsection{Chemicals}

Most chemicals were purchased from AppliChem, BioRad, Boehringer, Formedium, Fluka, Gerbu, Merck, Roche, Roth, Serva and Sigma. Other materials and instruments are mentioned in the following experimental methods.

\subsubsection{Buffers and media}

Table 2.1. List of buffers and media used in this study

\begin{tabular}{|c|c|}
\hline buffer / media & composition \\
\hline resuspension buffer & 20 mM HEPES, 500 mM NaCl, 8 mM imidazole, pH 7.4 \\
\hline extraction buffer & $\begin{array}{l}20 \mathrm{mM} \text { HEPES, } 500 \mathrm{mM} \mathrm{NaCl}, 8 \mathrm{mM} \text { imidazole, } 10 \% \\
\text { (w/v) sodium cholate, } \mathrm{pH} 7.4\end{array}$ \\
\hline wash buffer & 20 mM HEPES, $500 \mathrm{mM} \mathrm{NaCl}, 20 \mathrm{mM}$ imidazole $\mathrm{pH} 7.4$ \\
\hline elution buffer & $20 \mathrm{mM}$ HEPES, $500 \mathrm{mM} \mathrm{NaCl}, 400 \mathrm{mM}$ imidazole, $\mathrm{pH} 7.4$ \\
\hline dialysis buffer & $\begin{array}{l}20 \text { mM HEPES, } x \text { mM NaCl, } 1 \text { mM EDTA, } 1 \text { mM DTT, } \\
\text { pH } 7.4\end{array}$ \\
\hline liposome buffer & 20 mM HEPES, 150 mM KCl, 1 mM DTT, pH 7.4 \\
\hline disassembly buffer & $\begin{array}{l}50 \text { mM HEPES, } 20 \text { mM KAc, } 120 \text { mM KGlu, } 2 \text { mM ATP, } \\
5 \text { mM MgCl2, pH } 7.4\end{array}$ \\
\hline 3x sample buffer & $\begin{array}{l}1 \mathrm{M} \text { Tris, } 12 \%(\mathrm{w} / \mathrm{v}) \text { SDS, } 30 \%(\mathrm{w} / \mathrm{v} \text { ) Serva Blue, } 1 \mathrm{M} \\
\text { glycerol, } 0,4 \%(\mathrm{v} / \mathrm{v}) \beta \text {-mercaptoethanol }\end{array}$ \\
\hline
\end{tabular}


Table 1.1. continued

\begin{tabular}{|c|c|}
\hline 10x anode buffer & $2 \mathrm{M}$ Tris- $\mathrm{HCl}$ to $\mathrm{pH} 8.9$ \\
\hline 10x cathode buffer & $1 \mathrm{M}$ Tris, $1 \mathrm{M}$ Tricine \\
\hline gel buffer & 3 M Tris, $0.3 \%$ (w/v) SDS, pH 8.45 \\
\hline LB medium & $\begin{array}{l}1 \%(\mathrm{w} / \mathrm{v}) \text { tryptone, } 0.5 \%(\mathrm{w} / \mathrm{v}) \text { yeast extract, } \\
1 \%(\mathrm{w} / \mathrm{v}) \mathrm{NaCl}, \mathrm{pH} 7.0\end{array}$ \\
\hline LB agar plate & $1,8 \%(\mathrm{w} / \mathrm{v})$ agar dissolved in LB medium \\
\hline TB medium & $\begin{array}{l}1.2 \%(\mathrm{w} / \mathrm{v}) \text { tryptone, } 2.4 \%(\mathrm{w} / \mathrm{v}) \text { yeast extract, } \\
0.4 \%(\mathrm{w} / \mathrm{v}) \text { glycerin }\end{array}$ \\
\hline TB salts & 0.17 M KH2PO4, 0.72 M K2HPO4 \\
\hline blot transfer buffer & $\begin{array}{l}192 \mathrm{mM} \text { Glycin, } 25 \mathrm{mM} \text { Tris, } 0.04 \% \text { (w/v) SDS, } \\
20 \% \text { (v/v) methanol }\end{array}$ \\
\hline blocking buffer & $\begin{array}{l}0.1 \%(\mathrm{v} / \mathrm{v}) \text { Tween 20, } 20 \mathrm{mM} \text { Tris, } 150 \mathrm{mM} \mathrm{KCl} \text {, } \\
\text { pH } 7.5\end{array}$ \\
\hline blotto & $\begin{array}{l}5 \%(\mathrm{w} / \mathrm{v}) \text { nonfat dry milk powder dissolved in } \\
\text { blocking buffer }\end{array}$ \\
\hline TAE buffer & $40 \mathrm{mM}$ Tris, $20 \mathrm{mM}$ acetic acid, and $1 \mathrm{mM}$ EDTA \\
\hline
\end{tabular}

\subsubsection{DNA constructs}

The recombinant proteins used in this study are listed in Table 2.2. Previously, cDNAs encoding for neuronal SNAREs, $\alpha$-SNAP and NSF were derived from rat (Rattus norvegicus), bovine (Bos taurus) and Chinese hamster (Cricetulus griseus), respectively and were cloned into expression vectors. In addition, several neuronal SNARE variants were generated for this study using rat cDNA (see section 2.2.1).

\subsubsection{Synthetic genes}

Synthetic cDNA of synaptobrevin (syb, 1-116) S28C, $\Delta 84$ (the deletion mutant) was purchased from Genscript. The construct was optimized for expression in E.coli and was sub-cloned from pUC57 to pET28 vector (Novagen), which was necessary to obtain higher expression yields. A comparison of the mutated sequence to the wild type sequence can be found in Appendix A1. 
Table 2.2. List of DNA constructs used in this study

\begin{tabular}{|c|c|c|c|c|}
\hline gene & vector & insert & mutation & reference \\
\hline Stx1a & pET28a & $1-288$ & - & {$[27]$} \\
\cline { 2 - 5 } & pTXB1 & $1-288$ & - & this study \\
\cline { 2 - 5 } & pET28a & $183-288$ & - & {$[27]$} \\
\hline Snap25 & pET28a & $1-206$ & C84S, C85S, C90S, C92S & {$[144]$} \\
\cline { 2 - 5 } & pET28a & $1-206$ & C85S, C88S, C90S, C92S, S130C & {$[30]$} \\
\hline Vamp2 & pET28a & $1-116$ & - & {$[27]$} \\
\cline { 2 - 5 } & pET15b & $1-116$ & S28C & {$[27]$} \\
\cline { 2 - 5 } & pET28a & $1-116$ & $\Delta 84$ & {$[140]$} \\
\cline { 2 - 5 } & pET28a & $1-116$ & S28C, $\Delta 84$ & this study \\
\cline { 2 - 5 } & pET28a & $1-116$ & I45A, M46A & {$[116]$} \\
\cline { 2 - 5 } & pET28a & $1-116$ & S28C, I45A, M46A & this study \\
\cline { 2 - 5 } & pET28a & $1-96$ & S28C & {$[30]$} \\
\cline { 2 - 5 } & pET28a & $1-96$ & S28C, I45A, M46A & {$[116]$} \\
\cline { 2 - 5 } & pET28a & $1-52$ & S28C & this study \\
\cline { 2 - 5 } & pET28a & $1-52$ & S28C, I45A, M46A & this study \\
\cline { 2 - 5 } & pET28a & $1-65$ & S28C & this study \\
\cline { 2 - 5 } & pET28a & $1-65$ & S28C, I45A, M46A & this study \\
\cline { 2 - 5 } & pET28a & $1-87$ & S28C & this study \\
\cline { 2 - 5 } & pET28a & $1-87$ & S28C, I45A, M46A & this study \\
\cline { 2 - 5 } & pET28a & $49-96$ & - & {$[55]$} \\
\cline { 2 - 5 } & pET28a & $49-96$ & T79C & {$[55]$} \\
\hline NAPA & pET28a & $1-295$ & - & {$[99]$} \\
\hline NSF & pET28a & $1-744$ & - & \\
\hline
\end{tabular}

\subsection{Methods}

\subsubsection{Molecular cloning}

\subsubsection{Polymerase chain reaction (PCR)}

$50 \mu \mathrm{L}$ PCR reactions were prepared for standard molecular cloning. The components of the reaction and the PCR program for the thermal cycler (Biometra) are illustrated in Table 2.3. Phusion High-Fidelity DNA Polymerase, primers and the dNTP mix were purchased from Thermo Scientific, Sigma and NEB, respectively. 
Table 2.3. Polymerase chain reaction

\begin{tabular}{|c|c|c|c|c|}
\hline \multicolumn{2}{|c|}{ PCR mix } & & & \\
\hline volume $(\mu \mathrm{L})$ & component & \multicolumn{3}{|c|}{ thermal cycler program } \\
\hline 10 & buffer & step & temperature & duration \\
\hline 1 & $\mathrm{dNTP} \operatorname{mix}(10 \mathrm{mM})$ & 1 & $98^{\circ} \mathrm{C}$ & $30 \mathrm{~s}$ \\
\hline \multirow{2}{*}{2,5} & \multirow{2}{*}{$\begin{array}{l}\text { forward primer } \\
(10 \mu \mathrm{M})\end{array}$} & 2 & $98^{\circ} \mathrm{C}$ & $10 \mathrm{~s}$ \\
\hline & & 3 & $60^{\circ} \mathrm{C}$ & $15 \mathrm{~s}$ \\
\hline 2,5 & $\begin{array}{l}\text { reverse primer } \\
(10 \mu \mathrm{M})\end{array}$ & 4 & $72^{\circ} \mathrm{C}$ & $15 \mathrm{~s}$ \\
\hline 0,5 & DNA polymerase & 5 & \multicolumn{2}{|c|}{ go to step 2, repeat 29 times } \\
\hline 2 & template & 6 & $72^{\circ} \mathrm{C}$ & $10 \mathrm{~min}$ \\
\hline 31,5 & $\mathrm{ddH}_{2} \mathrm{O}$ & 7 & $16^{\circ} \mathrm{C}$ & finish \\
\hline 50 & total volume & & & \\
\hline
\end{tabular}

\subsubsection{DNA agarose gel electrophoresis}

PCR products were separated and visualized by 1,5 \% agarose gel electrophoresis [145]. Samples were mixed with 6x loading dye (Thermo Scientific) and their sizes were compared to DNA ladders, Gene Ruler $100 \mathrm{bp}$ or Gene Ruler $1 \mathrm{~kb}$ (Thermo Scientific). Electrophoresis was performed in TAE buffer at constant voltage (80 V). Agarose gel was then visualized by GelGreen ${ }^{\mathrm{TM}}$ nucleic acid gel stain (Biotium).

\subsubsection{Purification of PCR products}

After the size of a PCR product was validated via electrophoresis, it was purified following NucleoSpin ${ }^{\circledR}$ PCR Clean-up kit instructions (Macherey-Nagel). The purified nucleotide was dissolved in $\mathrm{ddH}_{2} \mathrm{O}$. It was further processed by the restriction enzymes and ligated into relevant vectors as an insert. 


\subsubsection{Restriction digestion}

The vector or the insert DNA were digested at $37^{\circ} \mathrm{C}$ for $1 \mathrm{~h}$. Mostly two restriction enzymes (double-digestion) were used in the one reaction mixture (see Table 2.4).

\subsubsection{Ligation}

Digested insert DNA was ligated to digested vector either at $16^{\circ} \mathrm{C}$ overnight or at room temperature for $1 \mathrm{~h}$ using T4 DNA Ligase (NEB, see Table 2.4).

Table 2.4. Restriction digestion and ligation reaction mixes

\begin{tabular}{|c|c|c|c|}
\hline \multicolumn{2}{|c|}{ restriction digestion mix } & \multicolumn{2}{|c|}{ ligation mix } \\
\hline volume $(\mu L)$ & component & volume $(\mu \mathrm{L})$ & component \\
\hline 3 & DNA ( 500 ng) & \multirow{2}{*}{2} & \multirow{2}{*}{ insert (1X, 20 ng) } \\
\hline 5 & Buffer 4 (NEB) & & \\
\hline & DUHET 4 (IVED) & 2 & vector $(4 X, 80 \mathrm{ng})$ \\
\hline 2,5 & NdeI (NEB) & 1 & T4 DNA Ligase Buffer \\
\hline 2,5 & Xhol (NEB) & 1 & T4 DNA Ligase (NEB) \\
\hline 0,5 & $\mathrm{BSA}(\mathrm{NEB})$ & 4 & $\mathrm{dd} \mathrm{H} 20$ \\
\hline 36,5 & $\mathrm{ddH} 20$ & \multirow{2}{*}{10} & \multirow{2}{*}{ total volume } \\
\hline 50 & total volume & & \\
\hline
\end{tabular}

\subsubsection{Bacterial transformation (heat shock method)}

Chemically competent E. coli XL1-Blue cells (Stratagene, $70 \mu \mathrm{L}$ ) were transformed using a protocol modified from [146]. Bacterial cells were thawed on ice for 5 minutes. Afterwards, they were mixed either with plasmid DNA (80 ng) or with ligation reaction mix $(10 \mu \mathrm{L}, \sim 80 \mathrm{ng})$ and incubated on ice for 10 minutes. Following a heat shock treatment at $42^{\circ} \mathrm{C}$ for 45 seconds, cells were placed back on ice for 2 minutes. Pre-heated LB medium (1 mL) was added and the mix was incubated shaking at $37^{\circ} \mathrm{C}$ for $40 \mathrm{~min}$. After this recovery incubation, bacteria were plated on 
Materials and Methods

relevant antibiotic containing agar plates and kept at $37^{\circ} \mathrm{C}$ overnight (e.g. Kanamycin containing plates for pET28a plasmid transformations).

\subsubsection{Plasmid purification and sequencing}

Freshly transformed bacteria were grown on agar plates. A single colony of these bacteria was picked and antibiotic supplemented LB medium was inoculated with it. The cells were grown shaking overnight at $37^{\circ} \mathrm{C}$. Next day, plasmid DNA was purified from harvested and lysed cells following NucleoBond® Xtra Midi kit instructions (Macherey-Nagel). The purified plasmid DNA was sequenced (Eurofins Genomics) and verified using NCBI BLASTn and BLASTp suites.

\subsubsection{Cloning strategies to generate neuronal SNARE variants}

Various neuronal SNARE constructs were generated for this study, using the molecular cloning methods that are explained above. Figure 2.1 illustrates two strategies for cloning a wild type syntaxin and a mutant synaptobrevin. A list of primers can be found in the Appendix.

\subsubsection{Protein expression and purification}

\subsubsection{Bacterial transformation (electroporation method)}

Electrocompetent E. coli BL21 DE3 cells (Stratagene, $70 \mu \mathrm{L}$ ) were transformed using a protocol modified from [147]. Bacterial cells were thawed on ice for 5 minutes. Bacteria and the plasmid DNA (80 ng) were mixed in an electroporation cuvette. The cuvette was then placed in between the electrodes of the electroporation device (MicroPulser, BioRad). A high voltage electrical pulse $(2.5 \mathrm{kV})$ was applied for $5 \mathrm{~s}$. Preheated LB medium ( $1 \mathrm{~mL}$ ) was added and the mix was incubated shaking at $37^{\circ} \mathrm{C}$ for $40 \mathrm{~min}$. After this recovery incubation, bacteria were plated on relevant antibiotic containing agar plates and grown at $37^{\circ} \mathrm{C}$ overnight (e.g. Kanamycin containing plates for pET28a plasmid transformations). 


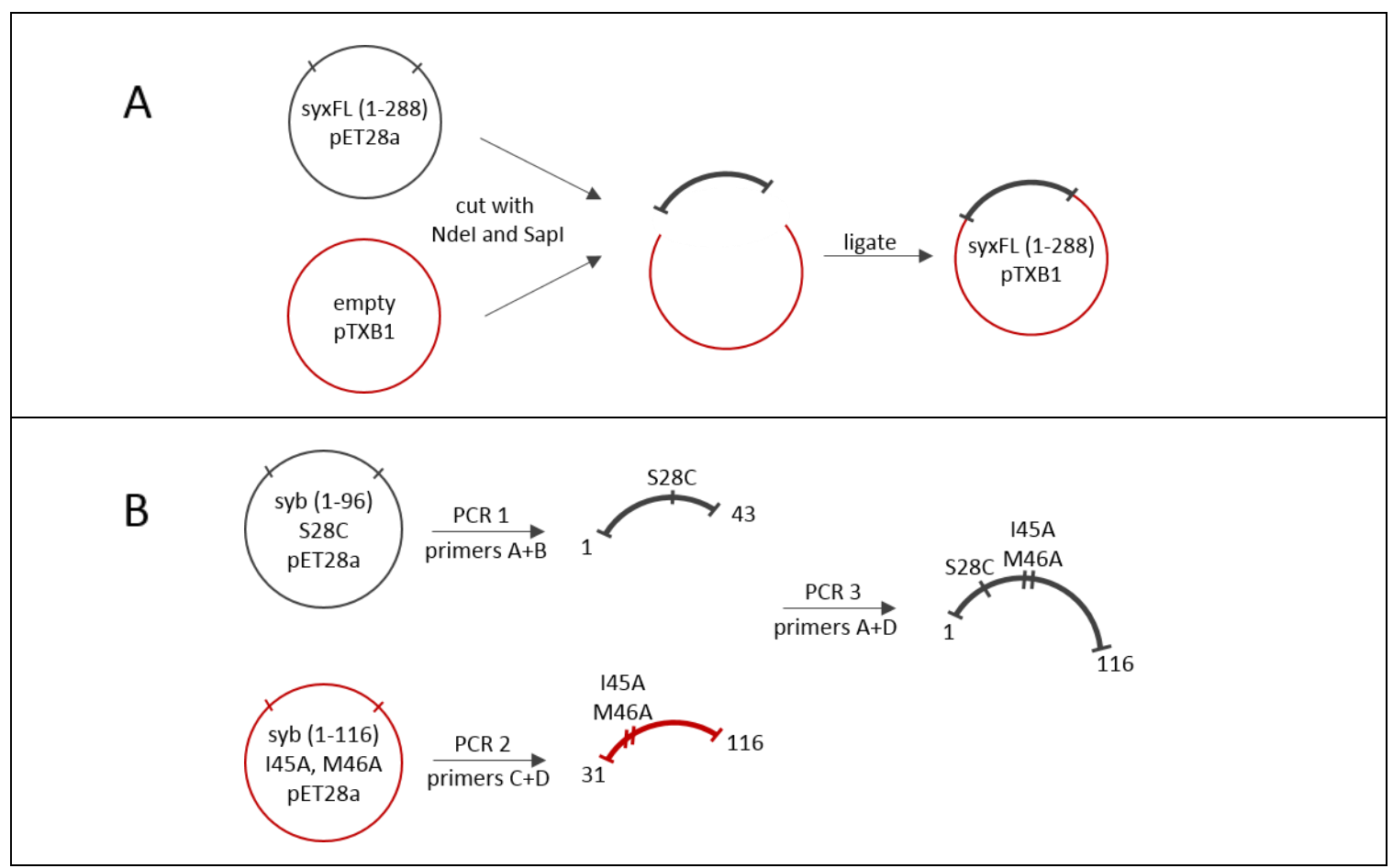

Figure 2.1. Schematic representations of the cloning strategies. A. sub-cloning of fulllength syntaxin (syxFL) from a pET28a to a pTXB1 vector using restriction enzymes Ndel and Sapl. B. The substitution mutant of synaptobrevin (syb $A A$ ), which can be fluorescently labeled, syb (1-116) S28C, 145A, M46A was generated using two different pET28a vectors as templates. An oligonucleotide carrying the single cysteine mutation and another with alanine substitutions were amplified from syb (1-96) S28C and syb (1116) $145 \mathrm{~A}, \mathrm{M} 46 \mathrm{~A}$ templates, respectively. These oligonucleotides were then used as templates to amplify the final insert syb (1-116) S28C, 145A, M46A.

\subsubsection{Recombinant protein expression in Escherichia coli}

Bacteria carrying the plasmid with a gene of interest were grown overnight in LB medium. This pre-culture was used next day to inoculate TB medium in which a large scale expression of the recombinant protein was induced. For each $500 \mathrm{~mL}$ of the TB culture, $450 \mathrm{~mL}$ TB medium, $50 \mathrm{~mL}$ TB salts, $500 \mu \mathrm{L}$ antibiotic (from 1000X stock, 50 $\mathrm{mg} / \mathrm{mL}$ Kanamycin or $100 \mathrm{mg} / \mathrm{mL}$ Ampicillin) and $10 \mathrm{~mL}$ of the pre-culture were mixed in a $2 \mathrm{~L}$ Erlenmeyer flask. Cells were grown shaking at $37^{\circ} \mathrm{C}$ until they reached OD600 0.8-1.0. At this density, bacteria were induced by adding IPTG (0.5 mM, f.c.). Soluble recombinant proteins were expressed in subsequent 3 hours, shaking at $37^{\circ} \mathrm{C}$; whereas membrane proteins were expressed overnight, shaking at $20^{\circ} \mathrm{C}$ to guarantee high yield and better protein folding. At the end of these incubations bacteria were 
Materials and Methods

harvested via centrifugation for $20 \mathrm{~min}$ at $4000 \mathrm{rpm}, 4^{\circ} \mathrm{C}$ (Beckman J6-MI). The pellet was resuspended in resuspension buffer $(100 \mathrm{~mL}$ buffer per $6 \mathrm{~L}$ bacterial culture pellet) and was kept at $-20^{\circ} \mathrm{C}$.

\subsubsection{Extraction and purification of SNARE monomers}

Almost all recombinant proteins were expressed with a His-tag via pET28 or pET15 vectors and purified by $\mathrm{Ni}^{2+}$-affinity chromatography. His-tag was removed by thrombin cleavage. Only a full-length wild type syntaxin construct (referred to as syxFL) was expressed using a pTXB1 vector with an intein and a chitin binding affinity tag. Inteins are protein splicing elements that can be induced to cleave themselves. Since the intein was followed by a chitin binding tag in this syxFL construct, chemically induced intein cleavage also removed the tag. Hence, via pTXB1 vector, there was no need for a protease cleaving the affinity tag. This strategy was chosen in order to prevent any proteolytic activity on syxFL N-terminal domain [66]. SyxFL was extracted and purified following the instructions of the IMPACT kit (NEB). All other His-tagged SNARE monomers were purified following the steps below:

- bacterial cell lysis

- protein extraction using detergents

- affinity purification using $\mathrm{Ni}^{2+}$-affinity chromatography

- His-tag cleavage with thrombin

- ion exchange chromatography

Bacterial cell suspensions were thawed and the same volume of extraction buffer was added ( $100 \mathrm{~mL}$ buffer per $6 \mathrm{~L}$ bacterial culture, total volume $\sim 200 \mathrm{~mL}$ ). Lysozyme ( 4 mg per $\mathrm{L}$ of initial culture), $\mathrm{MgCl}_{2}$ (1 mM, f.c.), DNaseI (tip of a spatula), protease inhibitor cocktail tablet (cOmplete, EDTA-free, Roche) were added to this suspension and incubated under stirring for 15 minutes at room temperature. Cells were lysed using a sonicator (Branson Sonifier 450, microtip limit, $50 \%$ duty cycle, 4 times 40 strokes of ultrasound). Urea (6 M, f.c.) was added to the cell lysate and dissolved by stirring for 15 minutes at room temperature. Solubilized protein was extracted via centrifugation at $13000 \mathrm{rpm}, 4^{\circ} \mathrm{C}$ for 1 hour (Sorvall RC6+ centrifuge, Thermo 
Scientific F146x250y rotor). The pellet was discarded and the supernatant was incubated under rotation for 2 hours with Ni-NTA agarose beads at $4^{\circ} \mathrm{C}$ (Qiagen, 12 $\mathrm{ml}$ beads per $6 \mathrm{~L}$ culture). His-tagged protein-bound beads were collected using an Econo-Column (BioRad). On the column, unspecifically bound proteins were washed off with the wash buffer. Next, using the elution buffer, which contains high amounts of imidazole and $\mathrm{NaCl}$, the protein of interest was eluted in 10 -mL-fractions. The fractions with high protein content were pooled together. Imidazole and $\mathrm{NaCl}$ were removed by dialysis overnight, and the His-tag was cleaved by adding thrombin ( 5 $\mathrm{mg} / \mathrm{mL}$ in $50 \%$ Glycerol $=1 \mathrm{U} / \mu \mathrm{L}, 100 \mu \mathrm{L}$ per $5 \mathrm{~mL}$ pooled fraction) into the dialysis tubing. Next day, the protein was further purified by ion exchange chromatography on an ÄKTA liquid chromatography system using a $\mathrm{NaCl}$ gradient (from $50 \mathrm{mM}$ to 500 $\mathrm{mM}$ ) for elution. Syntaxin and SNAP-25 were purified using anion exchanger (Mono Q column, GE Healthcare) whereas synaptobrevin was purified using cation exchanger (Mono S column, GE Healthcare). The purity of the elution fractions were evaluated by Tricine-SDS-PAGE [148]. The protein concentration of the most highly purified fractions was determined using a UV-Vis spectrophotometer (Nanodrop 1000). They were snap-frozen in liquid nitrogen and were kept at $-80^{\circ} \mathrm{C}$.

This procedure worked well for soluble cytoplasmic proteins like SNAP-25 and $\alpha$ SNAP. However it had to be modified slightly for the membrane anchored proteins like synaptobrevin and syntaxin. It was necessary to supplement all of the buffers used during the entire protocol with a detergent (e.g. $1 \%$ n-octyl- $\beta$-D-glucoside, Glycon). An example expression and purification profile of a synaptobrevin monomer is demonstrated with figure 2.2. Some exceptional constructs required further optimizations on the protocol. For an example, see Appendix A3.

\subsubsection{Purification of various SNARE complexes}

Several combinations of binary syx:SN25 and ternary syx:SN25:syb complexes were assembled in vitro from purified SNARE monomers (see Table 2.2). Monomers of choice were mixed and incubated overnight, rotating at $4^{\circ} \mathrm{C}$. To generate binary complexes of syx:SN25, the monomers were mixed with a molar ratio of 1:2. To 
generate ternary complexes, syb monomer was added in excess and the monomers were mixed with a 1:1:1,5 molar ratio. After overnight incubations, complexes were purified from unreacted monomers via ion exchange chromatography. A strong anion exchange column (mono Q 5/50 GL, GE Healthcare) was equilibrated in HEPES buffer (pH 7.4) with $1 \%$ CHAPS or with $1 \%$ n-octyl- $\beta$-D-glucoside (og). All complexes had at least one transmembrane domain and were reconstituted on liposomes. For fluorescence spectroscopic analyses, single or double labeled complexes served as fluorescent probes. These complexes were generated mixing fluorescently labeled monomers.

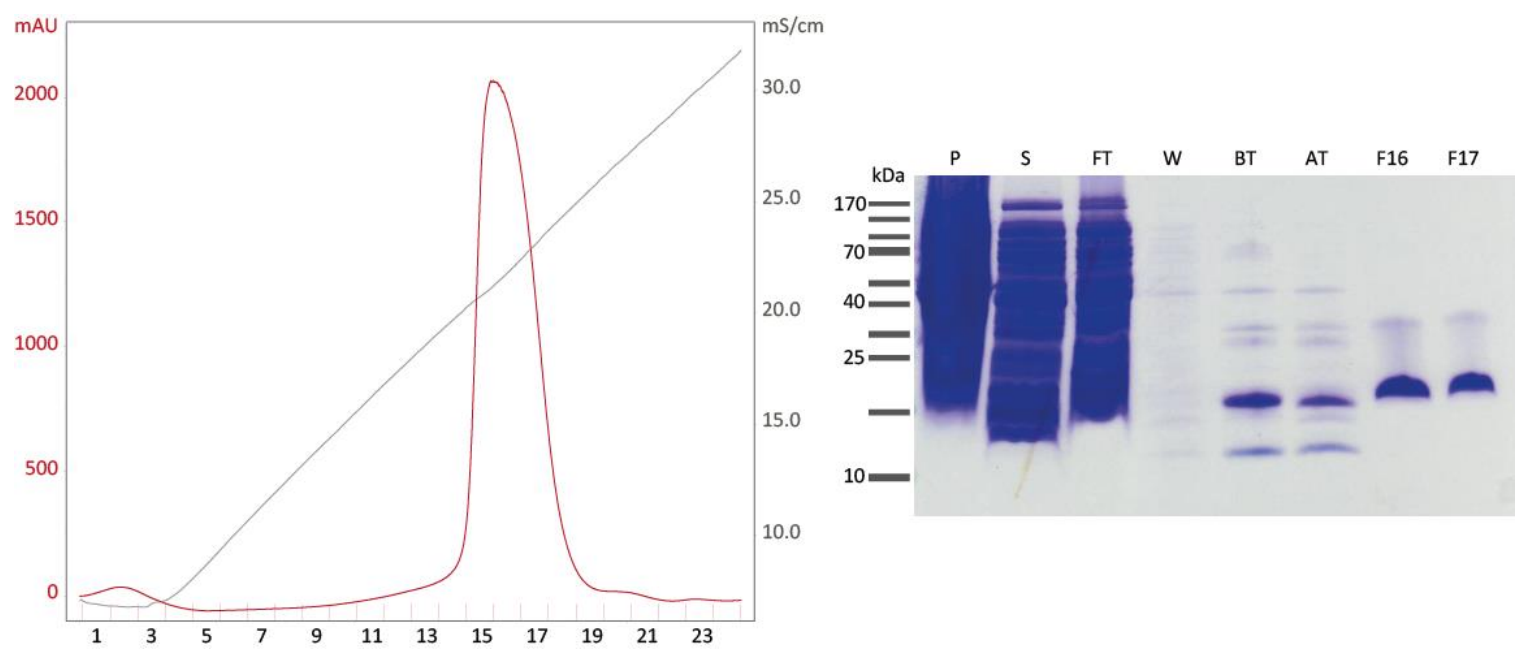

Figure 2.2. Expression and purification profile of syb (1-116) S28C, $\Delta 84$ (the deletion mutant). Left, ion exchange chromatography elution profile. The deletion mutant was eluted over a linear ionic strength gradient. Y-axis in red depicts the absorbance (mAU), while $y$-axis in gray shows the conductivity $(\mathrm{mS} / \mathrm{cm}) .2 \mathrm{~mL}$ elution fractions are numbered on the $x$-axis. The protein was eluted around $22 \mathrm{mS} / \mathrm{cm}$. Right, Tricine-SDS-PAGE analysis of various steps of the purification protocol. $P$, pellet and $S$, supernatant samples collected after centrifugation of the bacterial cell lysate. FT, flow through sample collected while Ni-NTA beads were recovered on an Econo-Column (BioRad), W, wash, BT, before thrombin, AT, after thrombin, F16 and F17, ion exchange elution fractions 16 and 17.

\subsubsection{Purification of the disassembly machinery}

In addition to neuronal SNARE monomers and complexes, two components of the disassembly machinery, $\alpha$-SNAP and NSF were purified and incorporated to the in 
vitro reconstitution system. An $\alpha$-SNAP construct from bovine cDNA was expressed and purified as explained in 2.2.2.2 and 2.2.2.3. Although, the NSF construct from Chinese hamster was expressed and the protein was extracted with a similar method, some changes were made in its purification protocol. All buffers used for the $\mathrm{Ni}^{2+}$ affinity purification step were supplemented with $10 \%$ Glycerol, 2 mM EDTA, 2 mM DTT, 0,5 mM ATP. After this step, instead of an ion exchange method, NSF hexamers were separated from the monomers using a gel filtration column (Sephadex 200 16/60, GE Healthcare) equilibrated in $50 \mathrm{mM}$ HEPES, pH 7.4, $200 \mathrm{mM} \mathrm{NaCl}, 10 \%$ Glycerol, $2 \mathrm{mM}$ EDTA, $2 \mathrm{mM}$ DTT, $0.5 \mathrm{mM}$ ATP. Snap-frozen aliquots of the protein samples were kept at $-80^{\circ} \mathrm{C}$ for future use.

\subsubsection{Preparation of SNARE-liposomes}

\subsubsection{Preparation of large liposomes}

Large liposomes (diameter, $100 \mathrm{~nm}$ ) were prepared following a recently developed protocol [38]. Phospholipids from porcine brain and cholesterol from ovine wool (Avanti Polar Lipids, Inc.) were dissolved in chloroform:methanol (2:1). In a pearshaped flask, PC:PE:PS:cholesterol were mixed with a molar ratio of 5:2:2:1 and an end concentration of $8 \mathrm{mM}$. The flask was fixed on a rotary evaporator and the solvent was evaporated by lowering the pressure gradually from 500 mbar to 50 mbar (BÜCHI Labortechnik). When a lipid film was formed on the walls of the flask, it was first dissolved in diethyl ether $(1,5 \mathrm{~mL})$ and then liposome buffer $(0,5 \mathrm{~mL})$ was added. The emulsion was sonicated using a thin tip, $50 \%$ duty cycles with low intensity pulses ( $3 \times x \quad 45$ s). Multilamellar vesicles were generated in various sizes by evaporating the ether using gradually decreasing air pressure from 500 torr to 50 torr (reverse-phase evaporation). When the ether was completely removed, the emulsion was re-adjusted to its end volume with liposome buffer when necessary. Finally, unilamellar large liposomes were prepared via serial extrusions using $0.4 \mu \mathrm{m}$ and $0.1 \mu \mathrm{m}$ polycarbonate membranes (Whatman). Table 2.5 provides an example for the composition of a lipid mix, which was calculated for an end volume of $500 \mu \mathrm{L}$. 
For lipid mixing and FCCS assays, various fluorescently labeled lipids were included in the lipid mixes, and liposomes were prepared as explained above. Liposomes with NBD/Rho labeled lipids were used for the lipid mixing assays (figure 2.3, a), whereas liposomes with OG-DHPE and TR-DHPE lipids were used for the FCCS assays (figure 2.4 , b). The fluorescent dyes were conjugated to either PE or PS. To keep the 5:2:2:1 molar ratio constant, less unlabeled PE and/or PS were added to the lipid mixes. Compositions of such lipid mixes can be found in Appendix A4.

Table 2.5. Composition of a lipid mix for large liposome preparations

\begin{tabular}{|c|c|c|c|c|c|}
\hline lipid & $\begin{array}{c}\text { lipid } \\
(\mathbf{\%})\end{array}$ & $\begin{array}{c}\text { MW } \\
(\mathbf{g} / \mathbf{m o l})\end{array}$ & $\begin{array}{c}\text { stock } \\
(\mathbf{m g} / \mathbf{m L})\end{array}$ & $\begin{array}{c}\mathbf{n} \\
(\boldsymbol{\mu m o l})\end{array}$ & $\begin{array}{c}\text { volume } \\
(\boldsymbol{\mu L} \mathbf{)}\end{array}$ \\
\hline PC & 50 & 770,94 & 25 & 2,00 & 61,68 \\
\hline PE & 20 & 746,06 & 10 & 0,80 & 59,68 \\
\hline PS & 20 & 812,05 & 10 & 0,80 & 64,96 \\
\hline chol & 10 & 386,66 & 10 & 0,40 & 15,47 \\
\hline total & - & - & - & 4,00 & 201,79 \\
\hline
\end{tabular}

\subsubsection{Protein reconstitution on large liposomes}

SNARE monomers and SNARE complexes were reconstituted on large liposomes by following a slightly modified direct reconstitution protocol [149]. A 'reconstitution mix' was prepared mixing the large liposomes with SNARE monomers or SNARE complexes purified in $1 \%$ og. This mix was supplemented with liposome buffer to reach a total volume and/or with detergent to suffice the correct R-value, which is an important parameter for this protocol. R-value is the ratio of the detergent concentration above critical micelle concentration (CMC) to the total detergent concentration. By adjusting the R-values of the reconstitution mixes above the CMC ( $\sim 17 \mathrm{mM}$ for og) and removing the detergent via dialysis, direct reconstitution of the proteins to the liposomes is achieved. A comprehensive explanation of how this method was developed can be found in [149].

For this study, reconstitution mixes with volumes of 350-500 $\mu \mathrm{L}$ were prepared. Rvalues were set to $R=1,5$ and to $R=2,0$, in order to reconstitute syb and SNARE complexes, respectively. The concentration of the liposome stock was set to 5,5 mM, as this was previously determined taking into account the lipid loss during the 
liposome preparation procedure [149]. If not stated otherwise, the lipid:protein ratio was set to 500:1 (see Table 2.6). The mix was injected into a dialysis cassette (SlideA-Lyzer Dialysis Cassettes, 2K MWCO, Thermo Scientific) and excess detergent was removed via two serial dialyses against liposome buffer at room temperature. Adsorbent beads were included in the first dialysis $(2 \mathrm{~g} / \mathrm{L}$, Bio-Beads SM-2 Adsorbents, Bio-Rad) which was done overnight. Next day, it was followed by a second dialysis for 3-4 hours. At the end of the dialyses, proteoliposomes were withdrawn out of the dialysis cassette using a syringe.

Table 2.6. Reconstitution mix to prepare synaptobrevin liposomes

The table shows the calculation sheet that was used to determine the composition of a reconstitution mix to prepare syb liposomes. The input parameters are shown with an asterisk whereas the output parameters are written in bold.

\begin{tabular}{|c|c|}
\hline MW of og $(\mathrm{g} / \mathrm{mol})$ & 292,4 \\
\hline og stock concentration $(\mathrm{mM})^{*}$ & 300,0 \\
\hline protein stock concentration $(\mu \mathrm{M})$ & 68,0 \\
\hline${\text { og concentration in protein stock }(\mathrm{mM})^{*}}^{*}$ & 34,0 \\
\hline liposome stock concentration $(\mathrm{mM})^{*}$ & 5,5 \\
\hline total volume $(\mu \mathrm{L})^{*}$ & 500,0 \\
\hline lipid:protein ratio* & 500,0 \\
\hline final lipid concentration $(\mathrm{mM})^{*}$ & 4,0 \\
\hline lipid volume $(\boldsymbol{\mu L})$ & $\mathbf{3 6 3 , 6}$ \\
\hline final concentration of protein $(\mu \mathrm{M})$ & 8,0 \\
\hline protein volume $(\boldsymbol{\mu L})$ & $\mathbf{5 8 , 8}$ \\
\hline (total detergent-cmc)/lipid $(\mathrm{R}-\mathrm{value})^{*}$ & 1,5 \\
\hline cmc in the presence of liposomes $(\mathrm{mM})^{*}$ & 17,0 \\
\hline final og concentration $(\mathrm{mM})$ & 23,0 \\
\hline Nog $(\mu m o l e)$ & 11,5 \\
\hline og derived from protein $(\mu \mathrm{mole})$ & 2,0 \\
\hline extra og stock required $(\boldsymbol{\mu L})$ & $\mathbf{3 1 , 7}$ \\
\hline extra buffer required $(\boldsymbol{\mu L})$ & $\mathbf{4 5 , 9}$ \\
\hline
\end{tabular}


Materials and Methods

\subsubsection{Fluorescence spectroscopy}

\subsubsection{Labeling proteins fluorescently}

Mutants of SNARE monomers were generated earlier with a single cysteine residue at desired positions, allowing for covalent labeling with thiol reactive fluorescent dyes. In this study, single cysteine mutants, syb S28C, syb T79C, and SN25 S130C, were used. Moreover, the syb layer mutants that can be fluorescently labeled, the deletion mutant, syb S28C $\Delta 84$ and the substitution mutant, syb S28C AA were generated. The proteins were labeled either with Oregon Green ${ }^{\circledR} 488$ iodoacetamide (referred to as OG) or Texas $\operatorname{Red}^{\circledR} \mathrm{C}_{5}$ bromoacetamide (referred to as TR). Both dyes were purchased from Molecular Probes and the labeling reaction was done following the instructions of the manufacturer. Briefly, the dye was dissolved in methanol and 5-6 moles of it was mixed with 1 mole of the protein in HEPES buffer ( $\mathrm{pH} 7.4$ ). Labeling mix was incubated rotating for 2 hours at room temperature. Unreacted dye was then separated using a disposable PD-10 desalting column (ready-to-use gel filtration columns packed with Sephadex G-25, GE Healthcare).

The concentration of the labeled protein was determined via a modified Bradford assay [150]. Serial dilutions of bovine serum albumin (BSA) stock $(0.1 \mathrm{mg} / \mathrm{mL})$ were prepared in $200 \mu \mathrm{L}$ volumes to obtain the following concentrations: $0,005 \mathrm{mg} / \mathrm{mL}$, 0,025 mg/mL, 0,050 mg/mL, 0,075 mg/mL, and 0,100 mg/mL. $800 \mu \mathrm{L}$ of Bradford working reagent (BioRad) was added to each of these dilutions. With the absorbance of each dilution at $\mathrm{OD}_{595}$ a standard curve was constructed. The concentration of the labeled protein was determined using the regression line of this curve.

Labeling efficiency was determined using the formula below:

$$
\frac{A x}{\varepsilon} \times \frac{M W \text { protein }}{C \text { protein }(\mathrm{mg} / \mathrm{mL})} \times 100
$$

where $A_{x}=$ the absorbance of the dye at the absorption maximum wavelength, $\varepsilon=$ molar extinction coefficient of the dye at the absorption maximum wavelength. 
Materials and Methods

\subsubsection{Förster resonance energy transfer}

Förster resonance energy transfer (FRET) is a commonly used method to investigate interactions between biomolecules in nm-scale. The excitation energy is transferred from an excited donor molecule to a ground state acceptor molecule, when there is spectral overlap between the emission spectrum of the donor and the absorption spectrum of the acceptor. Since this energy transfer occurs at distances of 1-10 nm, FRET is often chosen to study the interactions between proteins and/or lipids.

In this study, a popular fluorescence dequenching method for lipid mixing was chosen to study liposome fusion (figure 2.3 , a) $[38,131,151]$. Furthermore, a biochemical reconstitution system was developed to investigate SNARE complex dynamics on large liposomes via the FRET between labeled proteins (figure 2.3, b). Both bulk assays were conducted using the fluorescence spectrometer, Fluorolog 3 (Model FL322, Jobin Yvon), which was operated through manufacturer's Datamax software. Variations in the lamp intensity were corrected using the signal/reference (S/R) acquisition mode. A temperature bath was coupled to the spectrometer and all experiments were carried out at $37^{\circ} \mathrm{C}$ (if not stated otherwise) in quartz cuvettes mixing with a magnetic stirrer bar.

\section{Fluorescence dequenching method for lipid mixing}

NBD/Rho-labeled liposomes were prepared as explained in 2.2.3.1 using a lipid mix shown in Appendix A4. They were reconstituted with stabilized acceptor complexes and mixed with unlabeled syb liposomes ( $15 \mu \mathrm{L}$ of each liposome sample) in liposome buffer (figure 2.3, a). Each reaction had 1,2 mL total volume. Sample was excited at $460 \mathrm{~nm}$ and emission was collected at $538 \mathrm{~nm}$. Lipid mixing upon liposome fusion was monitored via the increase in NBD (donor) fluorescence. Once the reaction was complete, $12 \mu \mathrm{L}$ of $2 \%$ Triton X-100 (0,02 \%, f.c.) was added. This solubilized all liposomes and the resulting dequenching signal was taken as the maximum fluorescence $\left(F_{\max }\right)$. Normalized fluorescence (\%) was calculated using following formula: 
Materials and Methods

$$
\frac{F-F_{0}}{F_{\max }-F_{0}} \times 100=\% \text { lipid mixing }
$$

where $\mathrm{F}_{0}$ is initial fluorescence.

A FRET based assay for SNARE complex dynamics.

Recently developed assays were modified in order to monitor interactions of SNARE monomers in solution or reconstituted on liposomes using OG (donor) and TR (acceptor) labeled proteins (figure 2.3 , b) $[55,152]$. Changes in donor emission was probed for complex assembly or NSF-catalyzed disassembly (excitation at 488 and emission at $520 \mathrm{~nm}$ ). Fluorescence (F) was normalized with the initial fluorescence $\left(F_{0}\right)$. Various applications of this method are described in the results chapter.

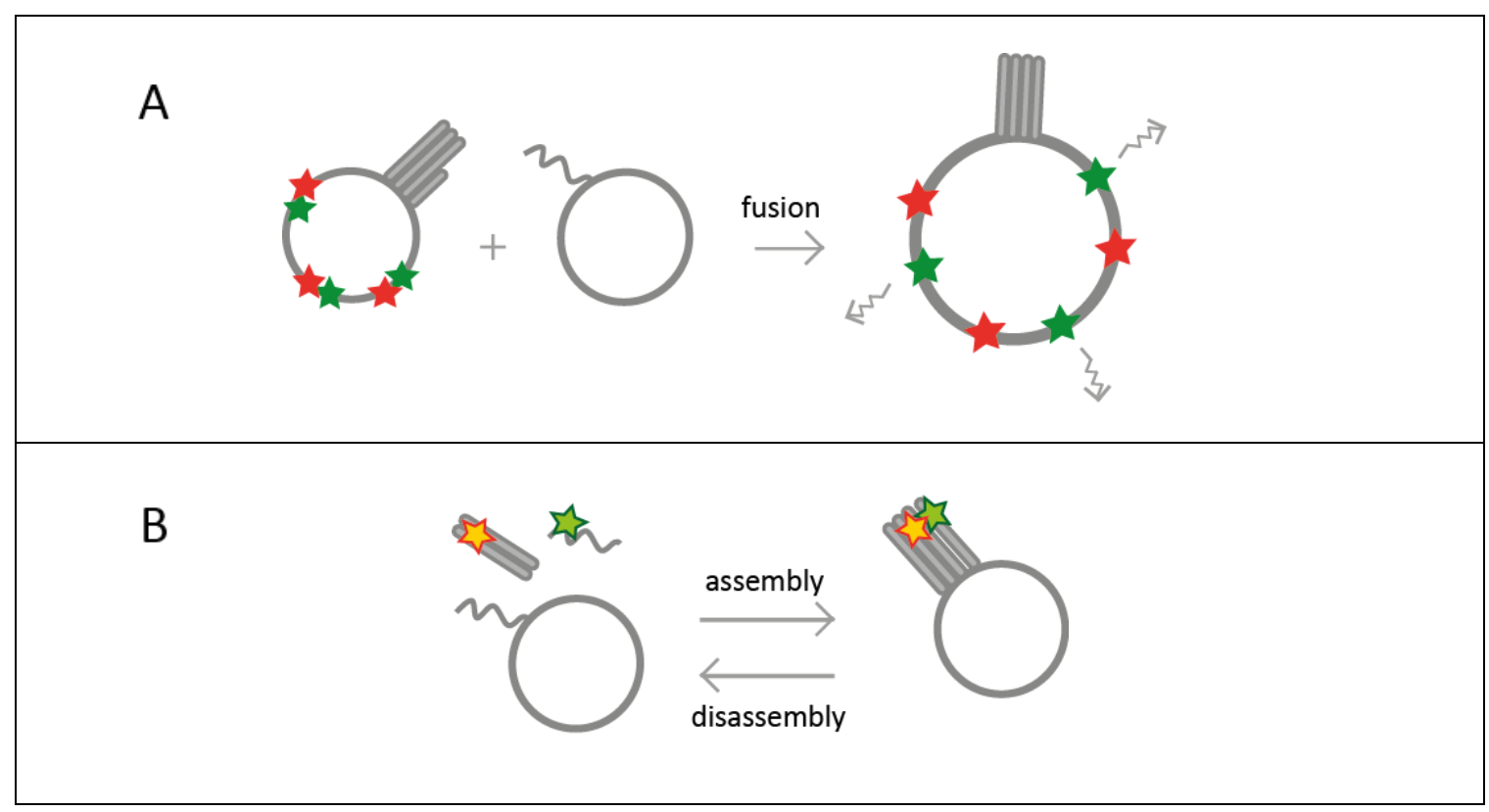

Figure 2.3. Illustrations of bulk FRET assays. A. NBD/Rho-labeled acceptor liposomes (with green and red stars) were mixed with unlabeled syb liposomes. Upon fusion, fluorescence probes were diluted and lipid mixing was measured as an increase in NBD emission (green star, the donor molecule). B. SNARE complex dynamics on liposomes were monitored by a FRET based assay. Mixing fluorescently labeled SNARE monomers developed a FRET signal which was reversed in NSF-catalyzed disassembly reactions. 


\subsubsection{Fluorescence Anisotropy}

In homogenous solutions, fluorescently labeled biomolecules have randomly oriented transition moments at their ground states. When exposed to linearly polarized light, only the ones with their transition moments parallel to the polarization vector are selectively excited. During the lifetime of this excited state, if the rotational diffusion of a molecule changes, depending on its size and the viscosity of the solvent, its emission will be depolarized. This is because its rotational diffusion has changed its transition moment. The changes in the rotational diffusion of a large molecule in a viscous solvent would be minimum. Hence the degree of polarization of the light it emits, which is described in terms of fluorescence anisotropy, will be higher [153]. On the contrary, a small molecule's fluorescence anisotropy will be lower.

Fluorescence anisotropy is used to track the changes in the rotational diffusion of biomolecules during the course of their interactions. In this study, anisotropy experiments were conducted to study SNARE complex assembly/disassembly cycles. The measurements were carried out in Fluorolog 3 (Model FL322, Jobin Yvon). Since the spectrometer is in T-configuration, vertically and horizontally polarized fluorescence intensities were collected simultaneously. Fluorescence anisotropy, r, is a dimensionless quantity that was calculated using the following equation:

$$
r=\frac{\left(I_{V V}-G I_{V H}\right)}{\left(I_{V V}+2 G I_{V H}\right)}
$$

where IVv and $I_{\mathrm{VH}}$ are the fluorescence intensities of vertically and horizontally polarized emissions of a sample which is excited with vertically polarized light. $G$ is an instrumental correction factor, which reflects the sensitivities of the detection systems for vertically and horizontally polarized emissions. It is calculated as follows:

$$
G=\frac{I_{H V}}{I_{H H}}
$$

where $\mathrm{IHV}_{\mathrm{HV}}$ and $\mathrm{I}_{\mathrm{H}}$ are the fluorescence intensities of vertically and horizontally polarized emissions of a sample which is excited with horizontally polarized light. 
All anisotropy recordings were done at $37^{\circ} \mathrm{C}$ in quartz cuvettes under mixing with a magnetic stir bar. OG or TR labeled proteins, reconstituted on liposomes, were used as fluorophores and the excitation/emission wavelengths were set to $488 / 520$ or $595 / 615$, respectively. Proteo-liposomes were prepared in the same way as was done for the bulk FRET assays as depicted in figure 2.3,b, however for anisotropy assays proteins were labeled with one single dye instead of a FRET pair. Prior to each set of experiment a new $\mathrm{G}$ factor was determined.

\subsubsection{Fluorescence correlation spectroscopy}

SNARE complex dynamics on liposomes and the resulting interactions of liposomes were investigated on the single-molecule/single-particle level via fluorescence correlation spectroscopy (FCS) and fluorescence cross-correlation spectroscopy (FCCS). These experiments were conducted using a confocal microscopy set-up in collaboration with Prof. Peter Jomo Walla's Group. Experimental details of the set-up are described in previous studies [154, 155].

Figure 2.4 illustrates two FCS assays developed for this study (see also figure 3.18 and figure 3.24). For all experiments, the total reaction volume was $300 \mu \mathrm{L}$. Acceptor liposomes were mixed with syb liposomes (either for protein-labeled samples or for lipid-labeled samples) in disassembly buffer ( $\mathrm{pH}$ 7.4) and incubated in a thermomixer, shaking at $1000 \mathrm{rpm}, 37^{\circ} \mathrm{C}$. In order to mix the liposomes with $1: 1$ molar ratio, the particle number for each liposome sample was determined and necessary dilutions were prepared. Usually $3 \mu \mathrm{L}$ of acceptor liposomes (1:100) were mixed with $3 \mu \mathrm{L}$ of syb liposomes (1:100). For each measurement, a $10 \mu \mathrm{L}$ droplet of the liposome mix was put onto a coverslip and measured for 10 x 15 s intervals. Each reaction was repeated three times. 


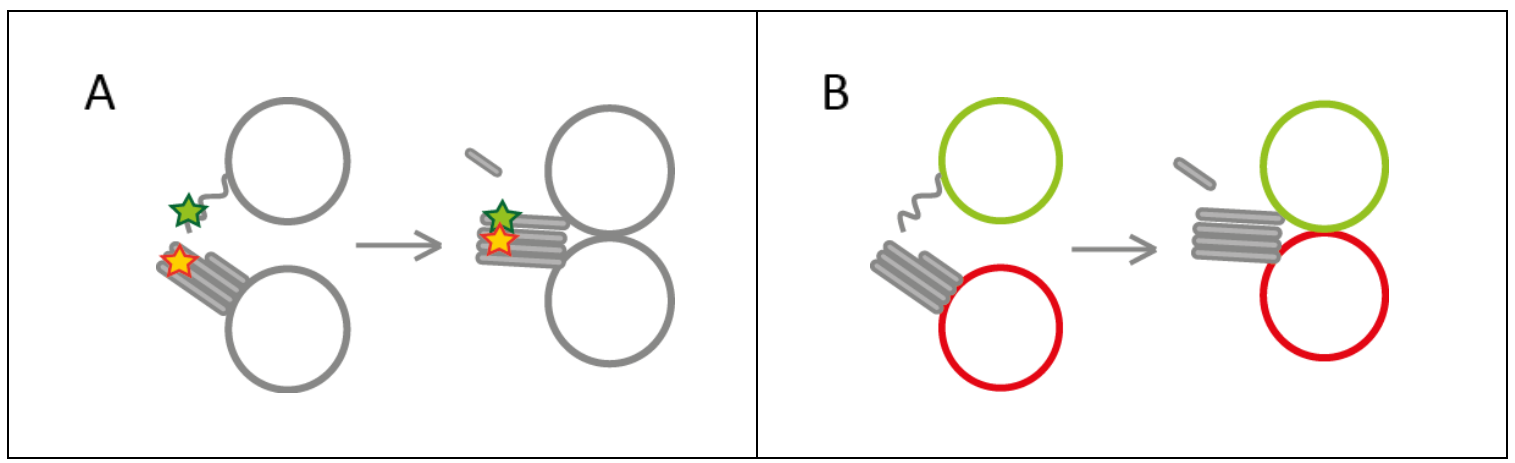

Figure 2.4. Illustrations of FCS assays. A, SNARE proteins were labeled with a FRET pair (TR labeled acceptor complex and OG labeled syb) and were reconstituted on large liposomes. SNARE complex assembly or disassembly processes were monitored via the changes in the donor lifetime (OG labeled syb). B, fluorescently labeled lipids were included in the lipid mixes when large liposomes were prepared. Texas Red-DHPE (TRDHPE) labeled large liposomes were reconstituted with acceptor complexes and mixed with Oregon Green-DHPE (OG-DHPE) labeled syb liposomes. Interactions of liposomes were assayed by fluorescence cross-correlation spectroscopy.

\subsubsection{Other methods}

\subsubsection{Density gradient centrifugation}

The soluble proteins that failed to get reconstituted on the large liposomes were separated from the proteoliposomes via density gradient centrifugation. This method also revealed the extent of protein reconstitution on liposomes. After the ultracentrifugation on a discontinuous Nycodenz ${ }^{\circledR}$ (Axis-Shield) gradient, liposomes are found in the top fractions. As illustrated in figure 2.5 proteins that are successfully reconstituted co-float with these liposomes, however proteins that fail to be incorporated do not get immobilized and stay in the bottom fractions.

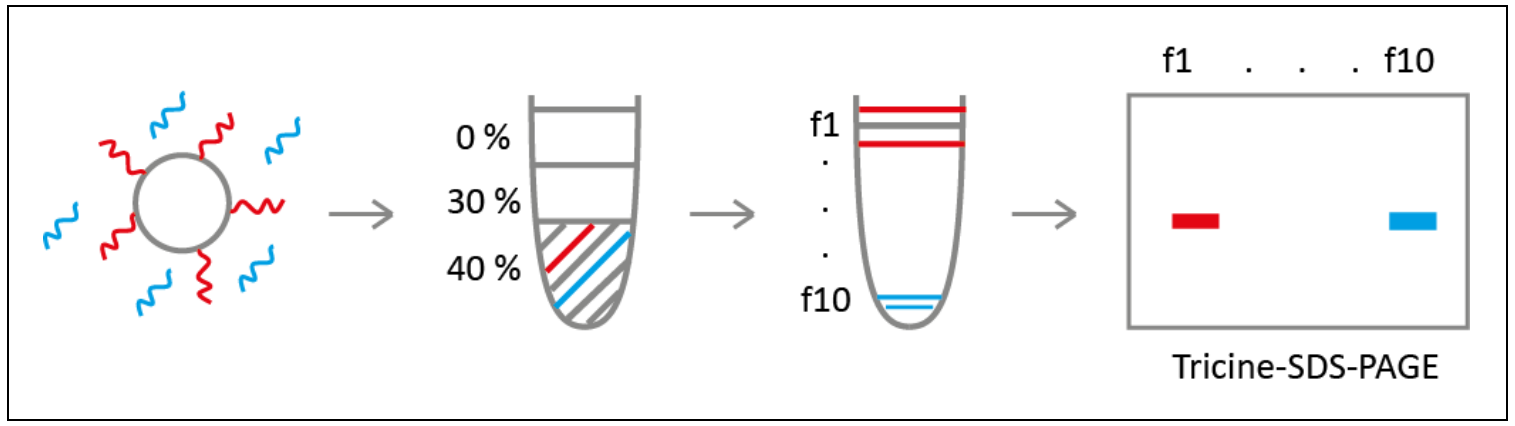

Figure 2.5. Illustration of the co-flotation assay. 
Materials and Methods

$80 \%(\mathrm{w} / \mathrm{v})$ and $30 \%(\mathrm{w} / \mathrm{v})$ Nycodenz stocks were prepared in liposome buffer. The proteoliposomes $(50 \mu \mathrm{L})$ were mixed with $80 \%$ Nycodenz solution $(50 \mu \mathrm{L})$ at the bottom of the centrifugation tube ( $250 \mu \mathrm{L}$ tube, Beckman). $30 \%$ Nycodenz solution (50 $\mu \mathrm{L}$ ) and liposome buffer (50 $\mu \mathrm{L}$ ) were applied gently on top of each layer. The discontinuous gradient was centrifuged at $55000 \mathrm{rpm}, 4^{\circ} \mathrm{C}$ for $90 \mathrm{~min}$ using S55-S rotor (Thermo Scientific) in Sorvall, Discovery, M150 SE, ultra-centrifuge. After the centrifugation, the fractions were analyzed via Tricine-SDS-PAGE. The gel was scanned for fluorescence (Fujifilm scanner, FLA-7000) when fluorescent proteins were reconstituted on liposomes as in figure 2.4, a. Unlabeled samples were analyzed via coomassie staining or western blotting.

\subsubsection{Tricine-SDS-PAGE}

Tricine-SDS-PAGE is an electrophoretic system with Tricine-Tris based buffers and is commonly used to separate proteins in the mass range 1-100 kDa. Since this system has lower acrylamide content, it is more suitable for proteins that are smaller than 30 $\mathrm{kDa}$ [148]. As most SNARE monomers and complexes are around this range, TricinSDS PAGE was used to analyze the purity of the SNARE samples.

Tricine gels were prepared using the composition shown in Table 2.7. Protein samples were mixed with $3 \mathrm{x}$ sample buffer, boiled briefly at $95^{\circ} \mathrm{C}$ and applied into the wells of a gel. In a BioRad running system, anode (outside) and cathode (inside) buffers were used to apply a constant voltage at $60 \mathrm{~V}$ (first 15 minutes) and $120 \mathrm{~V}$ (last 40 minutes). Proteins were then visualized by staining with Coomassie Brilliant Blue R-250 (0,2\% w/v) solution containing $50 \%(\mathrm{v} / \mathrm{v})$ methanol, $10 \%(\mathrm{v} / \mathrm{v})$ acetic acid. Two different de-staining solutions containing ethanol and acetic acid were used to remove the excess stain. 
Table 2.7. Tricine-SDS-PAGE gel compositions

\begin{tabular}{|c|c|c|}
\hline & collecting gel & running gel \\
\hline acrylamide & $200 \mu \mathrm{L}$ & $1,66 \mathrm{~mL}$ \\
\hline gel buffer & $375 \mu \mathrm{L}$ & $1,675 \mathrm{~mL}$ \\
\hline ddH 20 & $925 \mu \mathrm{L}$ & $570 \mu \mathrm{L}$ \\
\hline $\mathbf{5 0} \%$ glycerin & - & $1,06 \mathrm{~mL}$ \\
\hline TEMED & $2 \mu \mathrm{L}$ & $3 \mu \mathrm{L}$ \\
\hline $\mathbf{1 0} \%$ APS & $10 \mu \mathrm{L}$ & $25 \mu \mathrm{L}$ \\
\hline
\end{tabular}

\subsubsection{Western blotting}

Western blotting was performed using a protocol modified from [156]. Proteins separated by Tricin-SDS-PAGE were blotted to a nitrocellulose membrane $(0,2 \mu \mathrm{m}$, Perkin Elmer) via semi-dry blotting (Pegasus, Phase) under constant current (80 mA, 75 minutes). The membrane was then incubated in the blotto solution at room temperature for $1 \mathrm{~h}$ to block non-specific protein-protein interactions. After blocking, membrane was incubated with the primary antibody ( $\alpha$-SN25, Cl 71.1, Synaptic Systems) which was diluted (1:1000) in fresh blotto, shaking overnight at $4^{\circ} \mathrm{C}$. Next day, un-reacted antibody was washed off using the blocking buffer. After washing, secondary antibody (diluted 1:5000 in fresh blotto, goat $\alpha$-Mouse IgG HRP Conjugate, BioRad) was incubated on the membrane at room temperature for $1 \mathrm{~h}$. This solution was discarded and un-bound antibody was washed off using the blocking buffer. Lastly, the membrane was visualized via the reaction of secondary antibody with the HRP substrate (Western Lightning Plus-ECL, Perkin Elmer) in a chemi-luminescence detection chamber (LAS-1000, Fujifilm). 
Materials and Methods 


\section{Results}

\subsection{Strategies to reconstitute trans SNARE complexes in vitro}

The first section of the results chapter is about the strategies that were developed to reconstitute synaptic SNAREs in trans configurations between artificial membranes. Three reconstitution systems are introduced with different acceptor sites for synaptobrevin deletion mutant, syb $\Delta 84$ nucleation and SNARE complex formation. The first system involves the 2:1 complexes of syx:SN25 (will be referred to as the QSNARE complexes) as acceptor sites for synaptobrevin binding. In the second system, all three synaptic SNARE monomers were mixed in monomeric forms without any pre-assembled acceptor site. The third and the most effective system included stabilized acceptor complexes for synaptobrevin binding and trans complex assembly.

\subsubsection{First reconstitution system: Q-SNARE complexes as acceptor sites}

The key feature in the design of the first biochemical reconstitution system was the use of SNARE monomers in their most native forms possible, without any truncations and stabilizing syb (49-96) fragment [55]. For this, pre-assembled Q-SNARE complexes were chosen as acceptor sites for synaptobrevin binding. These complexes in almost all of the previous studies were generated via co-expression [70,131]. Since this approach might lead to co-purification of the additional hetero-oligomers of SNAP-25 and syntaxin (see section 1.1.2), in this study the Q-SNARE complexes were generated from their purified monomers (see section 2.2.2.4). The main components of this system were the large liposomes reconstituted either with Q-SNARE complexes or with the synaptobrevin deletion mutant, syb $\Delta 84$. In order to assemble 
Results

trans SNARE complexes, these two populations of large liposomes were mixed with each other. As it was previously shown on large liposomes, syb $\Delta 84$ exhibits an arrested fusion phenotype [38]. In this system it was tested whether this arrested fusion state might be reproduced starting from Q-SNARE complexes as preassembled acceptor sites. For a reference read-out, wild type synaptobrevin was also reconstituted on large liposomes and were mixed with Q-SNARE large liposomes to monitor the normal fusion reaction. Figure 3.1 depicts the schematics of the normal and arrested fusion reactions of the first reconstitution system.

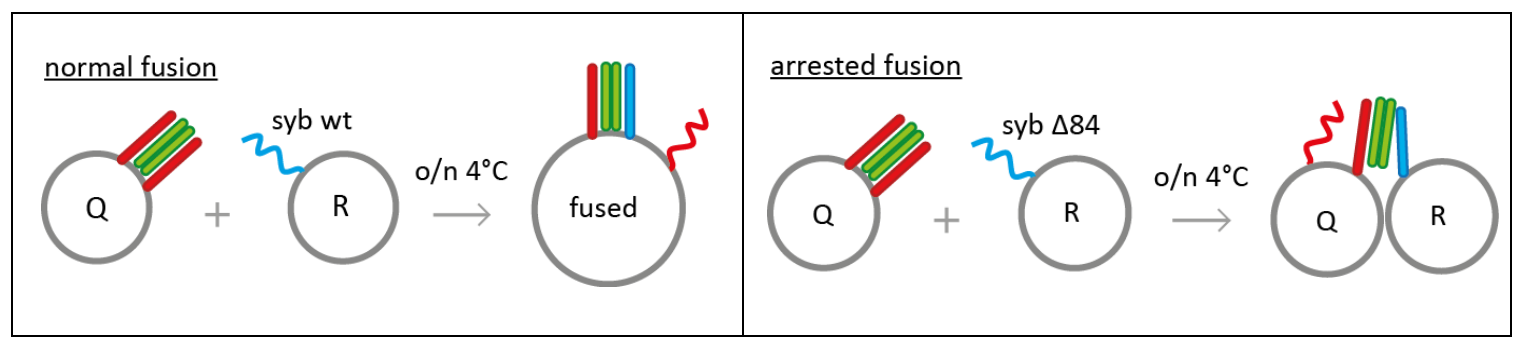

Figure 3.1. Schematic representation of the first reconstitution system. Q-SNARE complexes of syx (red) and SN25 (green) were reconstituted on large liposomes (liposome cartoons with Q). Q-liposomes were mixed with R-liposomes which were reconstituted with R-SNARE, synaptobrevin (blue). Left, normal fusion reaction scheme with complexes of wild type syb (syb wt) on fused liposomes. Right, arrested fusion reaction scheme with complexes of syb $\Delta 84$ on two docked Q- and R-liposomes.

One of the main goals of establishing a reconstitution system for trans complexes was to study whether these complexes were disassembled by NSF/ $\alpha$-SNAP. If the reactions had run as depicted in the schematic representations, cis and trans complexes would have formed on the fused and docked large liposomes, respectively. And the disassembly of both types of complexes would be compared via fluorescence anisotropy.

With this motivation, a previously developed anisotropy assay was modified and the disassembly of purified and fluorescently labeled ternary SNARE complexes was monitored on large liposomes [99]. This reaction served as the proof of principle demonstrating the disassembly of the membrane anchored cis SNARE complexes. A single cysteine variant of the cytoplasmic portion of synaptobrevin (cysteine substitution in the $28^{\text {th }}$ residue) was labeled with Oregon Green, and referred to as 
syb wt (1-96) OG. This labeled monomer was mixed with syntaxin and SNAP-25 and the single labeled ternary complexes were purified. The complexes were then reconstituted on large liposomes and mixed with $\alpha$-SNAP and NSF sequentially as the anisotropy of syb wt (1-96) OG was recorded (see figure 3.2). Synaptobrevin anisotropy displayed the rotational diffusion of the molecule, thus provided information whether it was found in a membrane anchored complex or free in solution. The initial increase in the anisotropy marked the binding of $\alpha$-SNAP, while the consecutive decrease was the result of NSF-driven disassembly. After the disassembly, the final anisotropy of syb wt (1-96) OG was lower than its initial anisotropy. This was an indication that the labeled protein dissociated off the membrane and became soluble. Excluding $\mathrm{Mg}^{2+}$ from this reaction prevented ATP hydrolysis and hence NSF-driven disassembly.
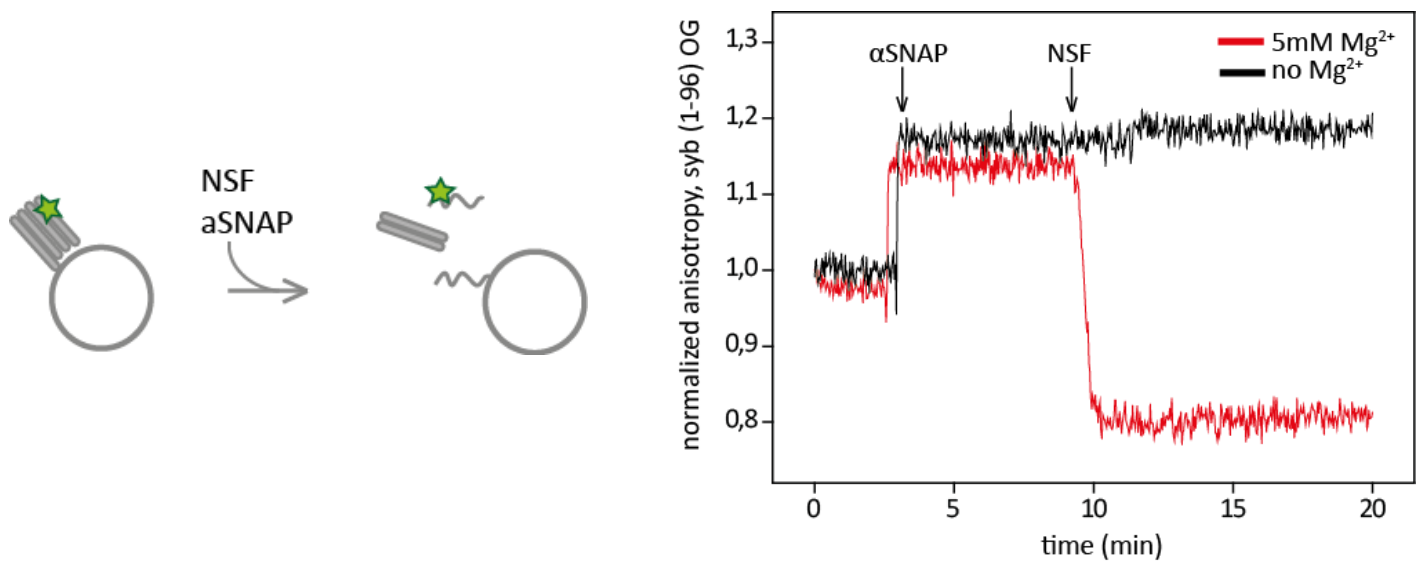

Figure 3.2. Single labeled ternary SNARE complexes on large liposomes are disassembled by NSF and $\boldsymbol{\alpha}$-SNAP. Ternary complexes of syb wt (1-96) OG, syx (183-288) and SN25 (1-206) were purified and were reconstituted on large liposomes via the transmembrane region of syx (183-288). Left, schematic representation of the disassembly reaction. Green star illustrates the Oregon Green label on synaptobrevin. Right, Anisotropy of syb wt (1-96) OG was monitored at $37^{\circ} \mathrm{C}$ and normalized to its initial anisotropy. Ternary complex liposomes $(10 \mu \mathrm{L})$ were mixed sequentially with $\alpha$-SNAP (1 $\mu \mathrm{M})$ and NSF (90 nM) in disassembly buffer $(600 \mu \mathrm{L})$.

Once the anisotropy assay was set up, normal fusion and arrested fusion reactions were performed to monitor cis or trans complex assembly reactions, respectively. Single cysteine variants of both syb wt and syb $\Delta 84$ were labeled with Texas Red (both at their $28^{\text {th }}$ cysteine residues) and were reconstituted on large liposomes. These 
Results

proteins were henceforth referred to as syb wt TR and syb $\Delta 84 \mathrm{TR}$. Their anisotropies were recorded as they were mixed with the Q-SNARE large liposomes in separate normal or arrested fusion reactions. However, only a gradual increase in the anisotropy was observed in either of the reactions. This indicated that labeled synaptobrevin on large liposomes binds to the Q-SNARE complexes at an extremely slow rate (data not shown). The reason for this expected outcome was because it was known that a prerequisite for synaptobrevin binding on the Q-SNARE complexes was the displacement of the second syntaxin (see section 1.1.2, [25] and [149]). Even if SNARE complexes were forming on large liposomes, it was happening very slowly in this system. Therefore, Q-SNARE and R-SNARE liposomes were mixed overnight, at $4^{\circ} \mathrm{C}$ for the pre-assembly of the cis and trans complexes. On the following day, the components of the disassembly machinery $\alpha$-SNAP and NSF were added and expected disassembly was monitored by the fluorescence anisotropy of syb wt TR and syb $\Delta 84$ TR, respectively (see figure 3.3).
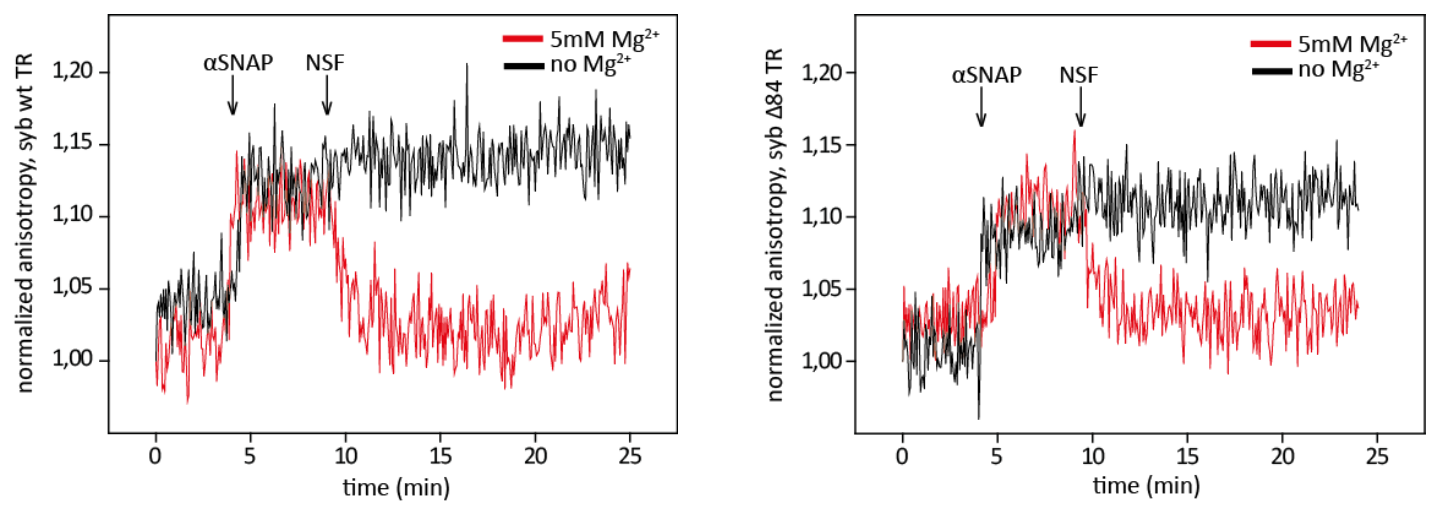

Figure 3.3. Compositions of the SNARE complexes that assembled overnight remained unclear. Q-liposomes $(10 \mu \mathrm{L})$ were mixed with R-liposomes $(10 \mu \mathrm{L})$ and incubated overnight at $4^{\circ} \mathrm{C}$. On the next day, the anisotropy of Texas Red labeled syb wt (left) and syb $\Delta 84$ (right) were monitored in the presence (red) or absence (black) of $\mathrm{Mg}^{2+}$ at $37^{\circ} \mathrm{C}$. $1 \mu \mathrm{M} \alpha$-SNAP and $90 \mathrm{nM}$ NSF were added sequentially to $600 \mu \mathrm{L}$ reactions as shown with arrows.

Addition of $\alpha$-SNAP increased the anisotropy of both syb wt and syb $\Delta 84$. Since it is known that $\alpha$-SNAP does not interact with free synaptobrevin [157], this increase indicates that $\alpha$-SNAP was binding to synaptobrevin in SNARE complexes that were formed on liposomes overnight. Addition of NSF decreased the anisotropy in $\mathrm{Mg}^{2+}$ 
dependent manner, indicating disassembly of the complexes. However, the composition of the SNARE complexes that assembled overnight remained unclear. If all syb wt TR or syb $\Delta 84 \mathrm{TR}$ were involved in complex formation, the anisotropy upon NSF addition would have decreased to a level lower than the initial anisotropy. This would correspond to the anisotropy of a disassembled synaptobrevin monomer. Nonetheless, such an anisotropy decrease was clearly not the case for either synaptobrevin variants.

After the experiments depicted with figure 3.2 and figure 3.3 were compared, the strategy to use Q-SNARE complexes as synaptobrevin acceptor sites was dismissed. The first reason was about the complex assembly rates being too slow either for the cis or trans SNARE complexes. The second and more technical reason was that the changes in anisotropy (see figure 3.3) were too small. This might have been due to the fact that membrane anchored labeled syb wt TR and syb $\Delta 84$ TR had given less anisotropy signal than soluble syb wt (1-96) OG.

\subsubsection{Second reconstitution system: two different approaches for monitoring cis and trans complex assembly}

The second reconstitution system tackled the above-mentioned complications in various ways. Firstly, in this system, the cis complex reaction involved liposomes that were reconstituted with purified ternary SNARE complexes, thus avoiding side reactions that may have occurred in SNARE assembly by fusion. Secondly, for the trans complex reaction, large liposomes were prepared containing syb $\Delta 84$ and syntaxin, respectively. To generate trans complexes these two populations of liposomes were mixed with soluble SNAP-25. Furthermore, reconstitution efficiency was monitored using a floatation gradient that allows for separation of the proteoliposomes from unincorporated protein. Figure 3.4 illustrates the cis and trans complex reactions of this second reconstitution system. 
Results

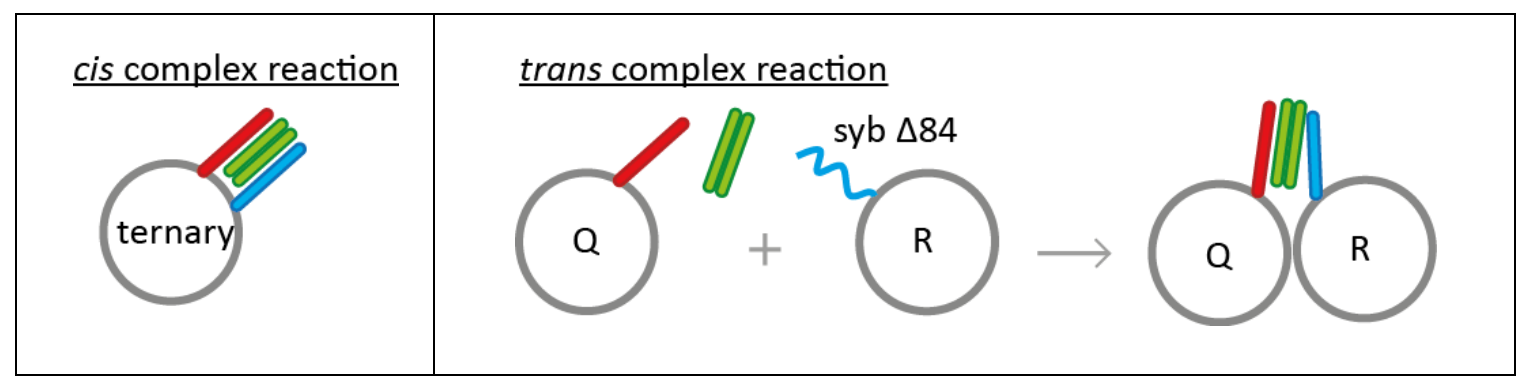

Figure 3.4. Schematic representation of the second reconstitution system. Syntaxin (red), SNAP-25 (green), synaptobrevin (blue). Left, ternary SNARE complexes, as cis complex models, were purified and reconstituted on large liposomes via the transmembrane regions of syntaxin and synaptobrevin. Right, the reaction scheme which is designed to generate trans SNARE complexes as syntaxin liposomes, syb $\Delta 84$ liposomes and soluble SNAP-25 are mixed in equimolar ratios.

In addition, instead of fluorescence anisotropy, Förster resonance energy transfer (FRET) was used to monitor SNARE assembly and disassembly. To this end, single cysteine variants of SNAP-25 and synaptobrevin (both wild type and $\Delta 84$ mutant) were labeled with Texas Red (acceptor dye) and Oregon Green (donor dye), respectively. These two probes were referred to as SN25 TR, syb wt OG and syb $\Delta 84$ OG. For labeling, cysteines were introduced in position 28 (synaptobrevin, same as experiments above) and 130 (SNAP-25). This pair was shown previously to result in a FRET signal as the SNAREs assemble into complexes that is reverted upon disassembly $[54,99]$.

Using these two fluorescence probes double labeled ternary SNARE complexes were purified and reconstituted on large liposomes. Tricine-SDS-PAGE (see figure 3.5, left) and floatation gradients (see figure 3.5, right) were employed to characterize the liposomes containing these labeled complexes. 


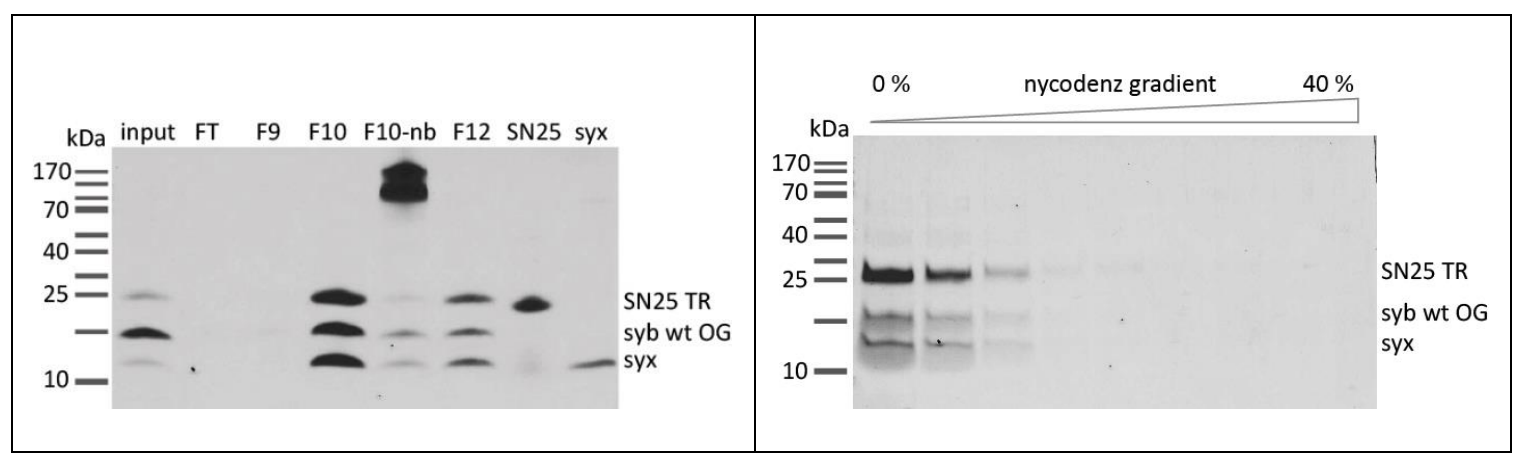

Figure 3.5. Double labeled ternary complexes were purified and reconstituted on large liposomes. Tricin-SDS-PAGE analyses of the purification profile (left) and the co-flotation assay (right). Gels were analyzed via coomassie staining. Left, syx (183-288), SN25 (1-206) TR, syb wt (1-116) OG were mixed overnight and on the next day the ternary complex was purified via an ion exchange column. Input, flow-through (FT) and the elution fractions from 9 to 12 (F9-F12) were analyzed. F10 was not boiled to visualize the SDS-resistant complex (F10-nb). Right, ternary complex liposomes were run over a discontinuous Nycodenz gradient. The fractions of this gradient after ultracentrifugation were analyzed.

In the cis complex reactions, NSF-driven disassembly of the double labeled ternary complexes on large liposomes was studied. The disassembly reaction components $\mathrm{NSF}, \alpha$-SNAP, ATP and $\mathrm{MgCl}_{2}$, each time leaving one of them out, were mixed with the ternary complex liposomes. Next, the missing component was added to start the reaction. Figure 3.6 shows a cis complex reaction, which was initiated by adding NSF. The same results were obtained when the disassembly was initiated with the addition of $\mathrm{ATP}, \mathrm{MgCl}_{2}$ or $\alpha$-SNAP (data not shown). As the ternary complexes were disassembled by NSF, FRET between SN25 TR and syb wt OG was lost and the disassembly was monitored with an increase in donor emission (syb wt OG).

These results indicated that using this approach disassembly of the cis complexes can be effectively measured. The next question was whether the trans complexes were disassembled in a similar way like the cis complexes. For this, a double labeled trans complex reaction was designed. Large liposomes that were reconstituted with syntaxin and Oregon Green labeled synaptobrevin deletion mutant, syb $\Delta 84$ OG were prepared. Two separate co-flotation assays verified that the proteins were successfully reconstituted on liposomes. Both syntaxin and syb $\Delta 84$ OG co-floated in the top liposome fractions of the discontinuous Nycodenz gradients (see figure 3.7). 


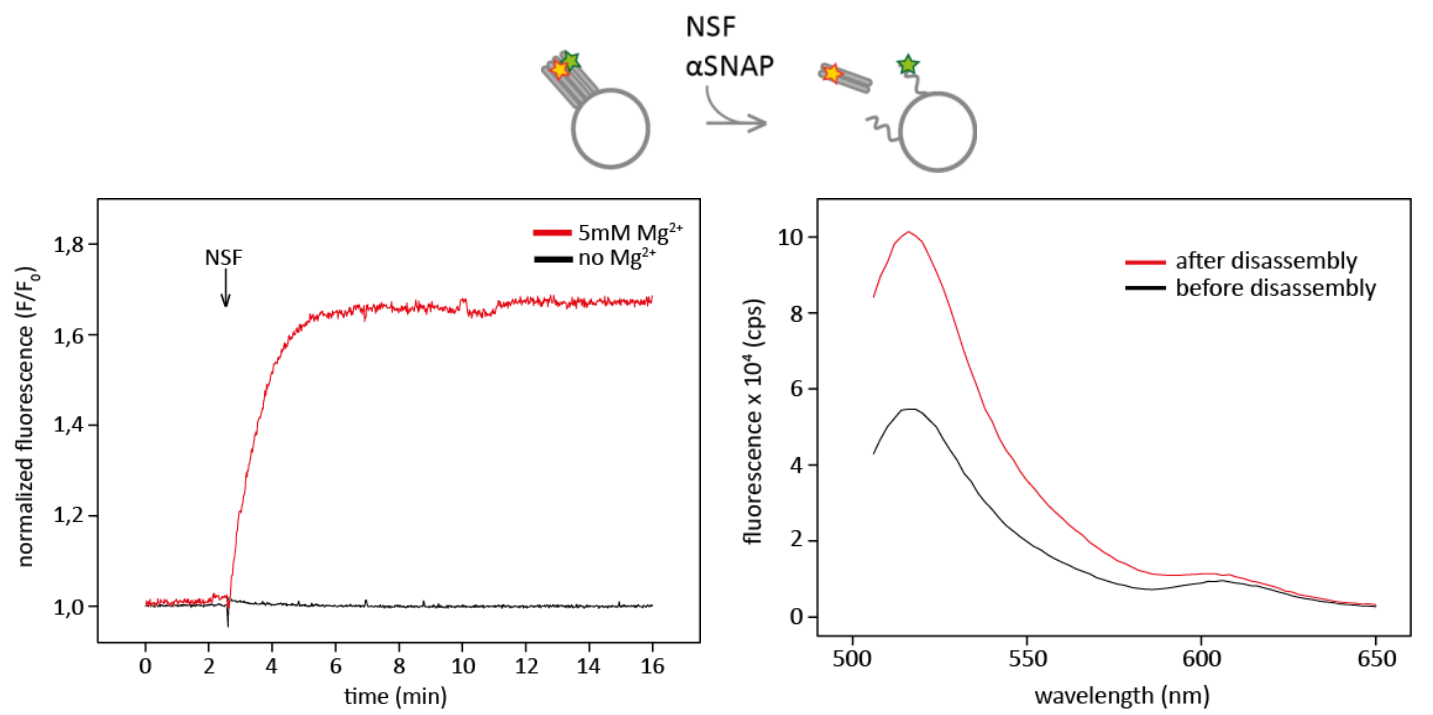

Figure 3.6. Double labeled ternary SNARE complexes on large liposomes are disassembled by NSF and $\boldsymbol{\alpha}$-SNAP. A schematic representation of the disassembly reaction of the double labeled ternary complexes is depicted on the top. Ternary complex liposomes $(10 \mu \mathrm{L})$ were mixed with $\alpha$-SNAP $(1 \mu \mathrm{M})$ in disassembly buffer $(600 \mu \mathrm{L})$. Disassembly was induced by adding NSF (90 nM). Left, fluorescence emission of syb wt OG (1-116) was monitored in the presence (red) or absence (black) of $\mathrm{Mg}^{2+}$. Fluorescence (F) was normalized with the initial fluorescence $\left(F_{0}\right)$. Right, Fluorescence emission spectrum of syb wt OG (1-116) before (black) and after (red) the disassembly reaction (excitation at $496 \mathrm{~nm}$ ).

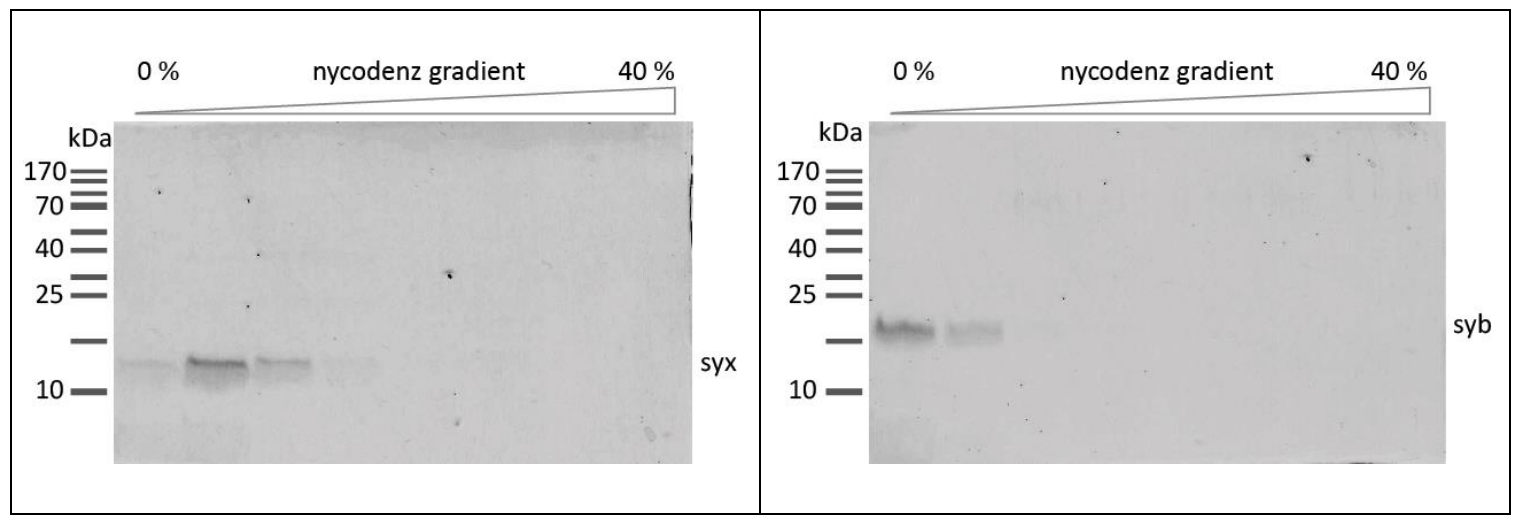

Figure 3.7. Syntaxin and syb $\Delta 84$ OG were reconstituted on large liposomes successfully. Syntaxin (left) and syb $\Delta 84$ OG liposomes (right) were run over a discontinuous Nycodenz gradient. After ultracentrifugation, the fractions of the gradients were analyzed via Tricin SDS-PAGE gels were visualized by coomassie staining. 
In the trans complex reactions, syntaxin liposomes and syb $\Delta 84$ OG liposomes were mixed with soluble SN25 TR in equimolar ratios. However, as the donor emission (syb $\triangle 84$ OG emission) was monitored, no FRET signal developed (data not shown). Since two SNARE monomers (syntaxin and synaptobrevin) were found on large liposomes, their diffusion rates in solution were much slower compared to soluble SN25 TR. Therefore, the rate of the trans complex assembly reaction was very low. To circumvent this problem, two types of liposomes and soluble SN25 TR were incubated overnight at $4^{\circ} \mathrm{C}$. Assuming that during this incubation some trans complexes had formed, it was tested whether they could be disassembled. On the following day, NSF and $\alpha$-SNAP were added to the trans complex reactions (see figure 3.8). Yet, emission of syb $\Delta 84$ OG emission did not reveal a disassembly trend similar to the one of syb wt OG (see figure 3.6). In fact, NSF addition did not change syb $\Delta 84$ OG emission to any extent.

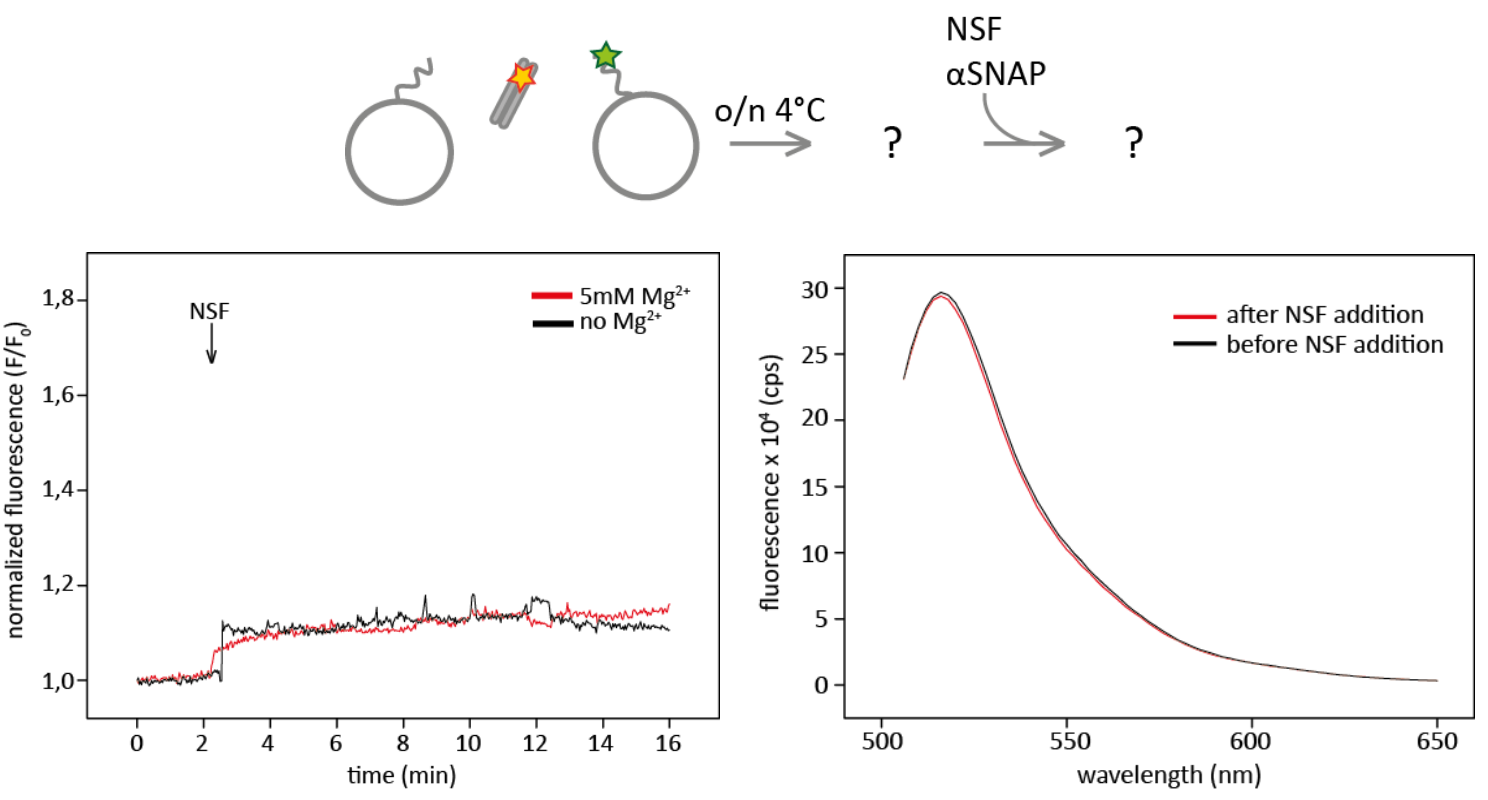

Figure 3.8. It was not possible to study the trans SNARE complex dynamics in the second reconstitution system. Syntaxin liposomes (10 $\mu \mathrm{L})$, SN25 TR monomers ( 130 nM), syb $\triangle 84$ OG liposomes $(10 \mu \mathrm{L})$ and $\alpha$-SNAP $(1 \mu \mathrm{M})$ were mixed in disassembly buffer $(600 \mu \mathrm{L})$. Disassembly was initiated by adding NSF $(90 \mathrm{nM})$. Left, fluorescence emission of syb $\Delta 84$ OG was monitored in the presence (red) or absence (black) of $\mathrm{Mg}^{2+}$. Fluorescence (F) was normalized with the initial fluorescence $\left(F_{0}\right)$. Right, fluorescence emission spectrum of syb $\Delta 84$ OG before (black) and after (red) NSF addition (excitation at $496 \mathrm{~nm}$ ). 
Results

The second reconstitution system failed to display trans complex dynamics in vitro. Even though the cis complex reaction was improved and another fluorescence spectroscopic method was implemented, whether any trans complexes assembled remained as an open question. Observations made in the first and the second biochemical reconstitution systems brought the necessity to monitor the trans complex assembly reaction real-time. Eventually, the main aim of these reconstitution systems was to display the trans complex assembly and their interactions with the regulatory factors in vitro. These realizations were taken into consideration while designing the third and the final reconstitution system.

\subsubsection{Final reconstitution system: using stabilized acceptor complexes}

Neither of the first two systems could reconstitute trans SNARE complex assembly kinetics as fast as the binding of synaptobrevin to the stabilized acceptor complexes (simply referred to as the acceptor complex) [55]. In order to increase the efficiency of trans complex assembly, the acceptor complexes were used to accelerate the binding of syb $\Delta 84$ several orders of magnitude [149]. In this system, the assembly and disassembly cycles for both cis and trans complexes were monitored in real-time by using the same FRET pair (SN25 TR and syb wt OG/syb $\Delta 84$ OG) that was introduced with the previous system. Once cis and trans complex reactions were set up, further fluorescence anisotropy or fluorescence cross correlation experiments were also developed to investigate the nature of the trans complexes.

The acceptor complexes were purified from the three monomers: syx (183-288), SN25 (1-206) and syb (49-96) with 1:1:1 stoichiometry and were reconstituted on large liposomes via the transmembrane domain of syntaxin. These large liposomes with the acceptor complexes were referred to as the acceptor liposomes. Binding of the soluble syb wt (1-96) on the acceptor liposomes generated cis complexes. This reference reaction was compared with the mixing of syb $\Delta 84$ liposomes with the acceptor liposomes, forming trans complexes between tightly docked large liposomes [38]. Below, figure 3.9 depicts the schematics of both cis and trans complex reactions that involve acceptor liposomes as the starting point. 
This final reconstitution system provided the medium to study the dynamics of the trans SNARE complexes successfully. The investigations, which were conducted using this system, are presented in the rest of this chapter.

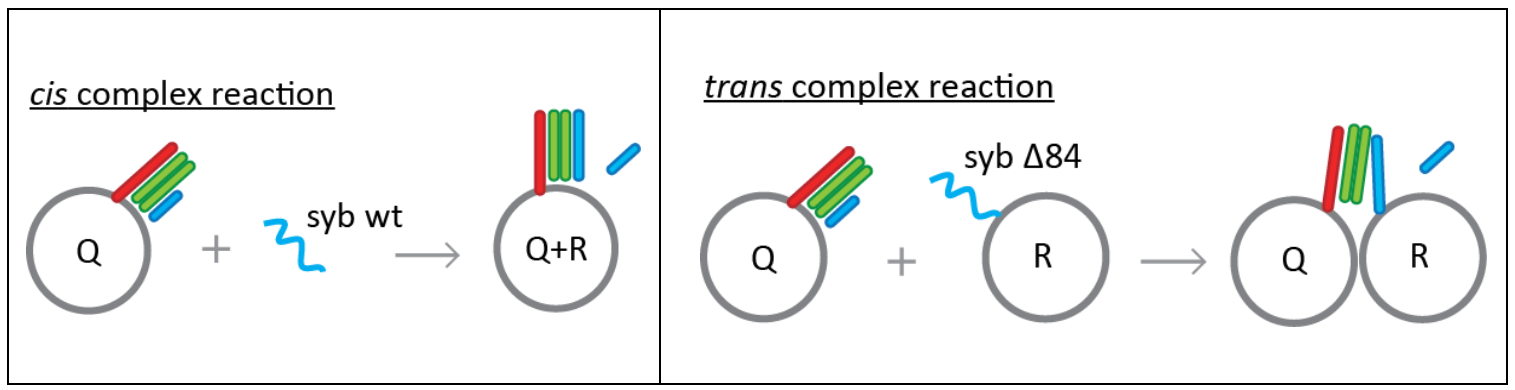

Figure 3.9. Schematic representation of the third reconstitution system. Syntaxin (red), SNAP-25 (green), synaptobrevin (blue). Left, reaction scheme for cis complexes on large liposomes. Acceptor complex liposomes (Q-liposomes) were mixed with soluble syb wt (1-96). Right, reaction scheme for trans complexes between two tightly docked liposomes. Acceptor liposomes were mixed with syb $\Delta 84$ liposomes. In both reaction schemes, soluble syb (49-96) fragment was depicted to be displaced off the Q-liposomes, with a cartoon of short blue rod.

\subsection{Trans SNARE complex dynamics: tightly zippering complexes}

This section presents the dynamics of the trans SNARE complexes that get arrested by syb $\Delta 84$ (see sections 1.3 and 3.1). The partial assembly of these complexes, their interaction with the disassembly machinery and whether they are susceptible to tetanus neurotoxin are among the problems that have been addressed here.

\subsubsection{The deletion mutant does not facilitate large liposome fusion}

It was imperative to verify the syb $\Delta 84$ phenotype before trans complexes were studied using the reconstitution system introduced above (see figure 3.9). For this purpose, fusion of large liposomes was monitored via a lipid mixing assay (see figure 3.10). NBD/Rho-labeled acceptor liposomes were mixed either with unlabeled syb wt liposomes or with unlabeled syb $\Delta 84$ liposomes. Expectedly, acceptor liposomes fused only to syb wt liposomes, whereas syb $\Delta 84$ liposomes failed to mediate fusion. In fact, the lipid mixing signal in the reaction of syb $\Delta 84$ liposomes overlapped with 
Results

the control reaction, where acceptor complexes were inhibited from fusing with excess soluble syb wt (1-96).

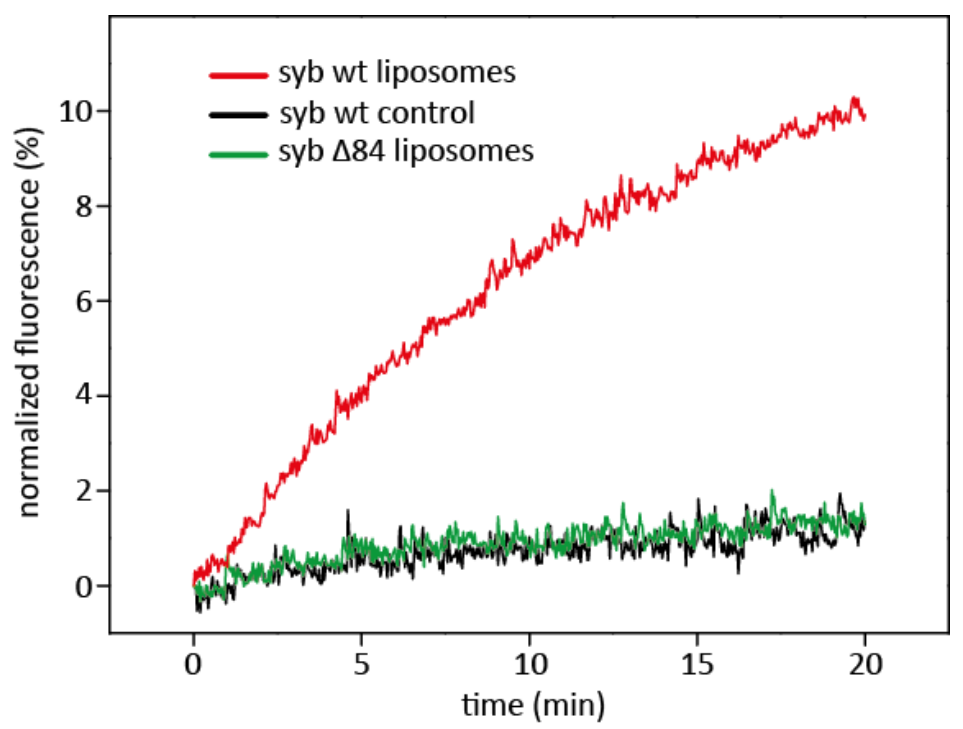

Figure 3.10. The deletion mutation prevents large liposome fusion. NBD/Rho-labeled acceptor liposomes $(15 \mu \mathrm{L})$ were mixed with synaptobrevin (syb) liposomes $(15 \mu \mathrm{L})$ in liposome buffer (total volume $1,2 \mathrm{~mL}$ ) at $37^{\circ} \mathrm{C}$. NBD fluorescence was monitored and normalized to its maximum fluorescence as explained earlier (in 2.2.4.2).

\subsubsection{NSF/ $\alpha$-SNAP-mediated disassembly of trans SNARE complexes}

The assembly of trans SNARE complexes was monitored via a FRET-based bulk assay. Here, cis SNARE complex assembly provided a reference reaction [55].

As Texas Red labeled SNAP-25 of the acceptor complex (the acceptor molecule) and Oregon Green labeled synaptobrevin (the donor molecule) took part in complex assembly, a FRET signal developed. This was observed with a decrease in the fluorescence emission of the donor. The same set of acceptor liposomes were the starting points to generate either cis complexes or trans complexes (see figure 3.11). Upon fast binding of soluble syb wt (1-96) OG to the acceptor complexes, the donor emission decreased rapidly. This indicated an expected assembly of the cis complexes [55]. Interestingly, when syb $\Delta 84$ liposomes were mixed with the acceptor liposomes, there was a decrease in the donor emission in this reaction as well. This marked the trans SNARE complex assembly between docking liposomes. The decrease in the donor emission in the trans complex reaction was slower than that of the cis complex 
reaction. Because both the donor and the acceptor molecules were reconstituted on large liposomes in the trans complex reaction, they had lower diffusion rates. Hence, trans complex formation happened slower than cis complex formation. Adding excess amounts of unlabeled syb wt (1-96) in the beginning of the reactions prevented the acceptor complexes from engaging into FRET-labeled cis or trans SNARE complex assembly (black traces in figure 3.11). This observation successfully verified that the change in the donor emission was a specific signal proving that the SNAREs interact reproducing the SNARE-dependent docking phenotype of syb $\Delta 84$.
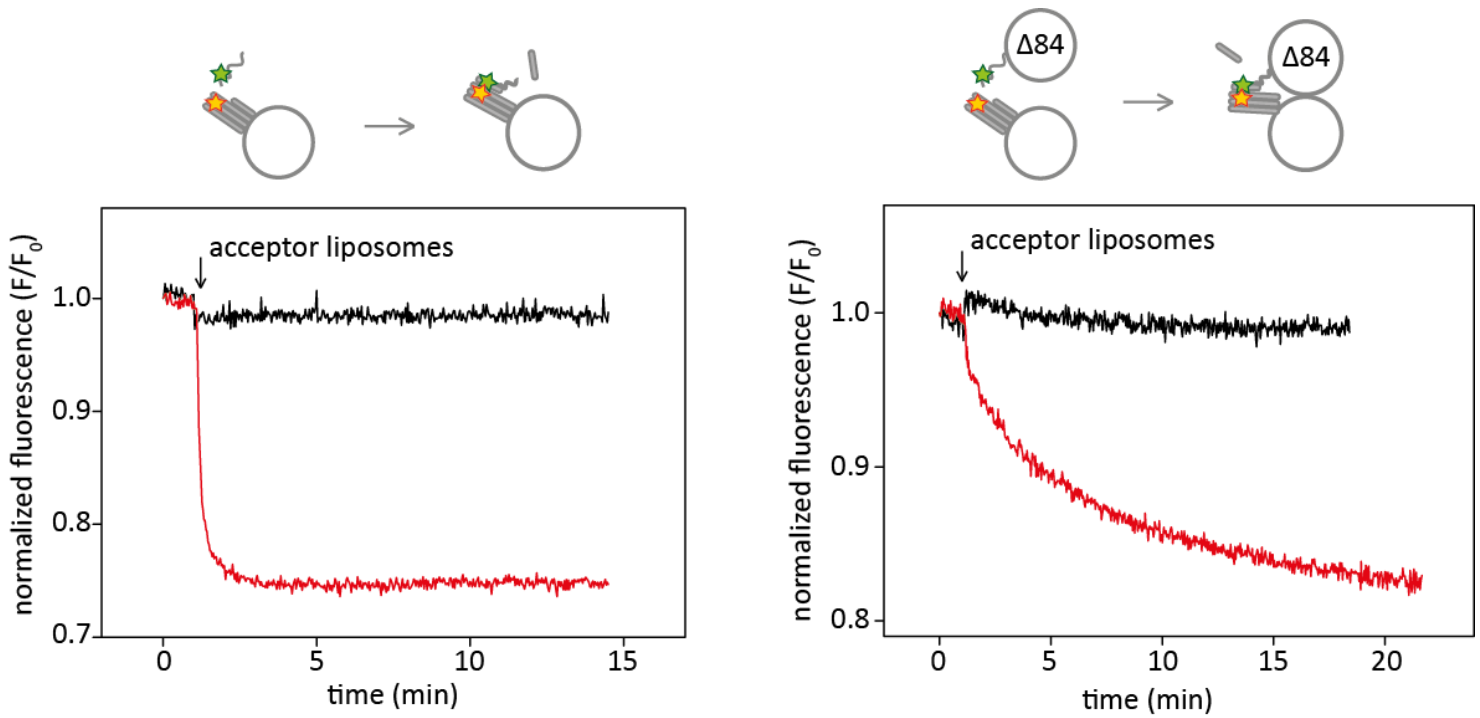

Figure 3.11. Assembly of the trans SNARE complexes between large liposomes was monitored with a bulk FRET-based assay. cis (left) and trans (right) complex assembly reactions on large liposomes were monitored via a FRET-based bulk assay. Texas Red labeled acceptor liposomes $(5 \mu \mathrm{L})$ were mixed either with Oregon Green labeled soluble syb wt (1-96, left) or with Oregon Green labeled syb $\Delta 84$ on liposomes ( $5 \mu \mathrm{L}$, right) in disassembly buffer (total volume $600 \mu \mathrm{L}$, red traces). Soluble syb wt (1-96) concentration was set such that its fluorescence counts matched the counts obtained from syb $\Delta 84$ liposomes. Fluorescence emission (F) of the donor (Oregon Green labeled synaptobrevin) was monitored and normalized to the initial fluorescence $\left(F_{0}\right)$. In control reactions excess (75-fold) unlabeled syb wt (1-96) was included (black traces). Red stars and green stars depict Texas Red label on SNAP-25 of the acceptor complex and Oregon Green label on synaptobrevin, respectively. The rod cartoon represents the short syb (49-96) fragment of the acceptor complex which is displaced off the membrane upon complex formation. 
Results

Once this reconstitution system for trans SNARE complex assembly was established, it was used as a standard medium to investigate how pre-fusion SNARE complexes are regulated. First, the interactions between these complexes and the disassembly machinery were characterized. The FRET-based assay was exploited to test whether NSF disassembled the trans SNARE complexes.

After Texas Red and Oregon Green labeled cis and trans complexes were generated as explained above, $\alpha$-SNAP and NSF were added. The disassembly of the cis SNARE complexes was examined as reference reaction [99]. Expectedly, as cis complexes were disassembled by NSF/ $\alpha$-SNAP, the donor emission increased rapidly and recovered its initial counts (see figure 3.12, left panel). Remarkably, when trans SNARE complexes were mixed with $\alpha$-SNAP and NSF, the donor emission recovered similarly (see figure 3.12, right panel). Such a recovery in the donor emission was not observed without $\mathrm{MgCl}_{2}$, when NSF was inactive. These observations provided direct evidence on NSF-mediated disassembly of the trans SNARE complexes on tightly docked large liposomes.

The efficiencies of protein reconstitutions in acceptor liposome and syb $\Delta 84$ liposome preparations were assessed by co-flotation assays. These assays did not reveal any proteins in the soluble fractions (data not shown). However, it was still necessary to demonstrate that the FRET signal in the assembly and disassembly reactions resulted only from the proteins found on the membranes. This was addressed by repeating the trans SNARE complex assembly reaction with "cleared" liposomes. Acceptor liposomes and syb $\Delta 84$ liposomes were run separately over discontinuous Nycodenz gradients (see 2.2.5.1). After the ultracentrifugation, proteoliposomes were collected from the top fractions. This step separated them from the proteins that had failed to be reconstituted. Figure 3.13 compares two assembly reactions performed using the liposomes before and after ultracentrifugations. As the donor emissions of syb $\Delta 84$ liposomes were overlaid, there were no differences in the FRET signals. This experiment confirmed that the liposome preparations were not contaminated with soluble proteins. 

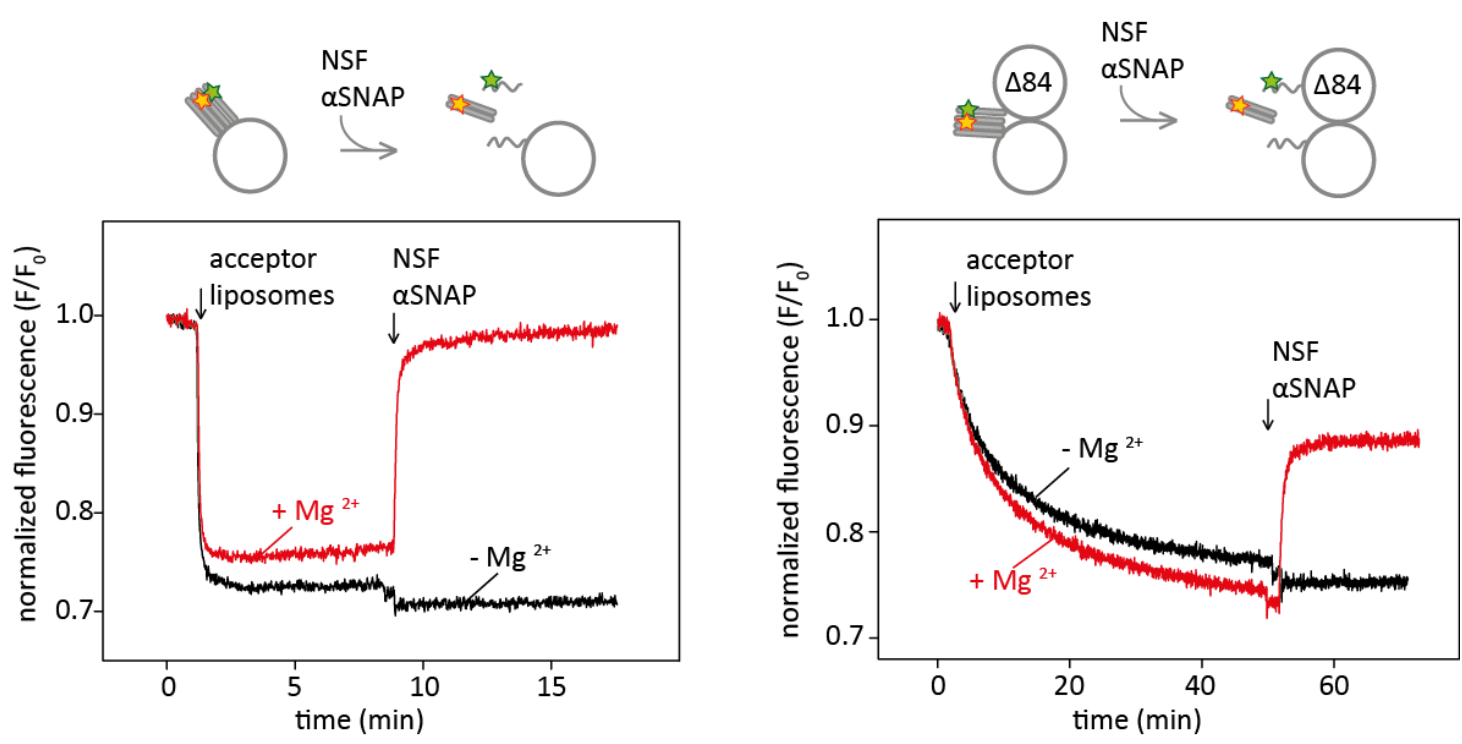

Figure 3.12. NSF/ $\alpha$-SNAP disassembles trans SNARE complexes on large liposomes. Interactions of the cis (left) and trans (right) SNARE complexes with the disassembly machinery were monitored via a FRET-based bulk assay. After Texas Red and Oregon Green labeled cis and trans complexes were generated as in figure 3.11, $\alpha$-SNAP $(1 \mu \mathrm{M})$ and NSF (60 nM) were added. Red traces represent the reactions in disassembly buffer (total volume $600 \mu \mathrm{L}$ ) which contained $5 \mathrm{mM} \mathrm{MgCl}_{2}$, whereas the black traces represent the control reactions repeated without $\mathrm{MgCl}_{2}$. Red stars and green stars depict Texas Red label on SNAP-25 of the acceptor complex and Oregon Green label on synaptobrevin, respectively. Fluorescence of the donor (Oregon Green labeled synaptobrevin) was normalized to its initial value $\left(F / F_{0}\right)$.

Up to this point, a truncated syntaxin construct (syx, 183-288) was used to develop an ideal reconstitution system and to monitor the assembly/disassembly cycles of SNARE complexes. This construct has a SNARE motif and a transmembrane domain, but lacks the regulatory $\mathrm{N}$-terminal domain of syntaxin (see figure 1.2 and, [27]). Earlier, using fluorescence anisotropy, it was shown that synaptobrevin binds identically to the acceptor complexes formed either with syx (183-288) or with full length syntaxin, syx (1-288) [149]. That is, the N-terminal domain of syntaxin was not involved in synaptobrevin binding to the acceptor complexes. In the light of this observation, the following experiments in this study were done using syx (183-288). Nevertheless, it was also tested whether the acceptor complexes of syx (183-288) behaved similarly to the acceptor complexes of syx (1-288). 
Results

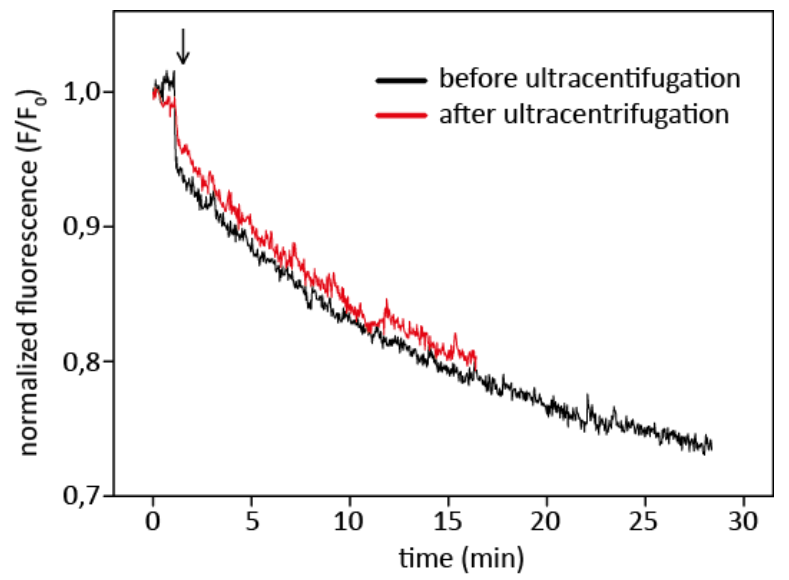

Figure 3.13. FRET signal marks the interactions of the proteins that are reconstituted on the membranes. Acceptor liposomes reconstituted with Texas Red labeled acceptor complexes $(5 \mu \mathrm{L})$ were mixed with donor liposomes reconstituted with Oregon Green labeled syb $\Delta 84(5 \mu \mathrm{L}$ ) in disassembly buffer (total volume $600 \mu \mathrm{L}$, black trace). The same reaction was repeated with proteo-liposomes that were collected from the top fractions of the Nycodenz gradients after ultracentrifugations. The arrow shows the time point where acceptor liposomes were added as in figure 3.11, right panel. Fluorescence of the donor (Oregon Green labeled synaptobrevin) was normalized to its initial value ( $\left.F / F_{0}\right)$.

For this, full-length acceptor complexes were generated using syx (1-288), Texas Red labeled SNAP-25 (1-206) and the stabilizing fragment of syb (49-96). They were reconstituted on large liposomes via the transmembrane domain of syx (1-288). The FRET-based bulk assays to monitor cis and trans complexes were repeated using this Texas Red labeled full-length acceptor complex. The assembly reactions of the fulllength cis and trans SNARE complexes with syx (1-288) were very similar to their truncated counterparts (see figure 3.14, left). These complexes were further tested for NSF-driven disassembly. Addition of NSF and $\alpha$-SNAP showed that full-length cis and trans complexes were disassembled almost identically to their truncated counterparts (see figure 3.14, right). These results demonstrated that the N-terminal domain of syntaxin does not have any effects on the disassembly of the trans SNARE complexes. Moreover, the acceptor complexes containing either the truncated or the full-length syntaxin monomer behave very similarly in this reconstitution system. Hence, the proceeding experiments were performed using the truncated variant which was more practical to prepare. 

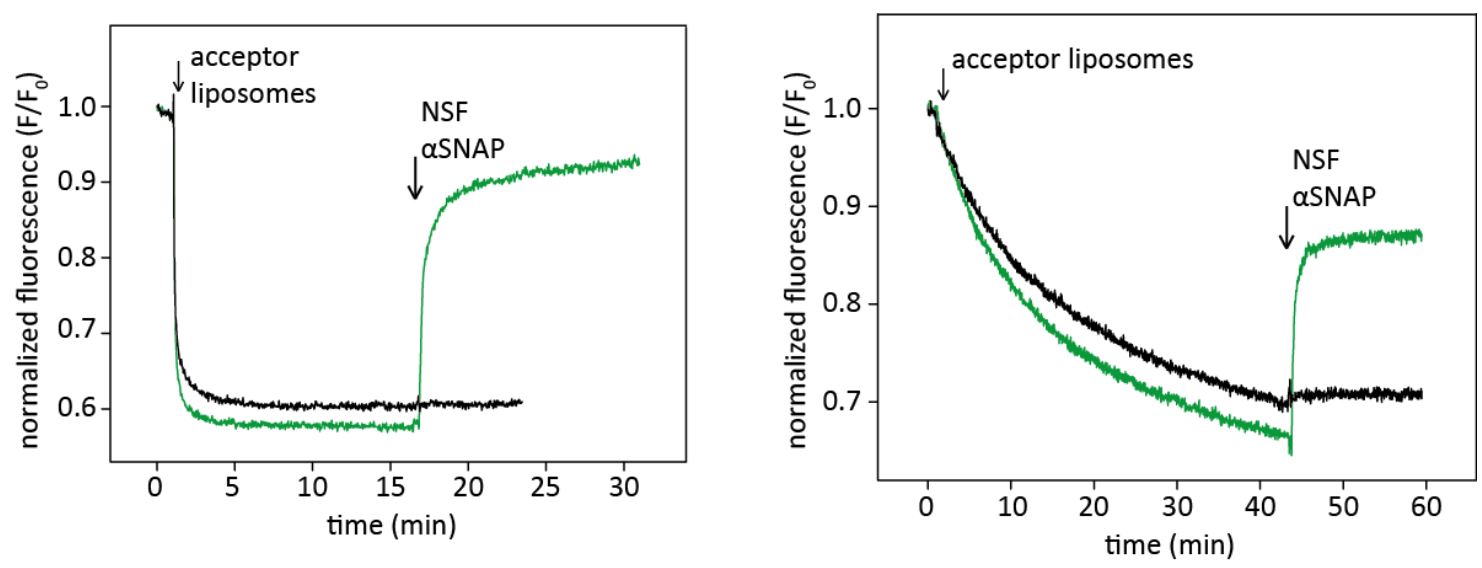

Figure 3.14. NSF/ $\alpha$-SNAP disassembles full-length trans SNARE complexes that contain the $\mathbf{N}$-terminal domain of syntaxin. Texas Red labeled acceptor complexes were purified with syx (1-288) and were reconstituted on large liposomes. These acceptor liposomes (5 $\mu \mathrm{L}$ ) were mixed either with Oregon Green labeled syb wt (1-96, left) or with Oregon Green labeled syb $\Delta 84$ liposomes ( $5 \mu \mathrm{L}$, right). After cis and trans complexes assembled, $\alpha$-SNAP $(1 \mu \mathrm{M})$ and NSF (60 nM) were added. Green traces represent the reactions in disassembly buffer (total volume $600 \mu \mathrm{L}$ ) which contained $5 \mathrm{mM} \mathrm{MgCl}_{2}$, whereas the black traces represent the control reactions repeated without $\mathrm{MgCl}_{2}$. Fluorescence of the donor (Oregon Green labeled synaptobrevin) was normalized to its initial value ( $\left.F / F_{0}\right)$.

\subsubsection{SNARE monomers do not re-assemble after trans complex disassembly}

Trans SNARE complexes residing between tightly docked liposomes were shown to be disassembled by NSF. With the following set of experiments, this system was characterized further. The first question asked was regarding the disassembled monomers of the trans complexes. Were these monomers re-assembling into fresh trans complexes? If this was the case, it would create a state of disassembly/reassembly equilibrium, which would explain why the donor emission did not recover fully after the trans complex disassembly (see figure 3.12 and figure 3.14, right panels). To answer this question, the trans complex disassembly reaction was repeated in the disassembly buffer which was supplemented with 10 mM EDTA (2folds of $\mathrm{MgCl}_{2}$ concentration). This experiment showed once again that it was possible to inhibit NSF with EDTA because it requires Mg-ATP hydrolysis to disassemble trans SNARE complexes (see figure 3.15, left). In the next experiment, NSF was inhibited with EDTA after it disassembled the trans complexes (see figure 3.15, right). If the monomers were re-assembling into fresh trans complexes, they would yield a FRET 
Results

signal (decrease in donor emission). However, at this time window when disassembly was stopped with EDTA, donor emission remained unchanged showing no indications of re-assembly.
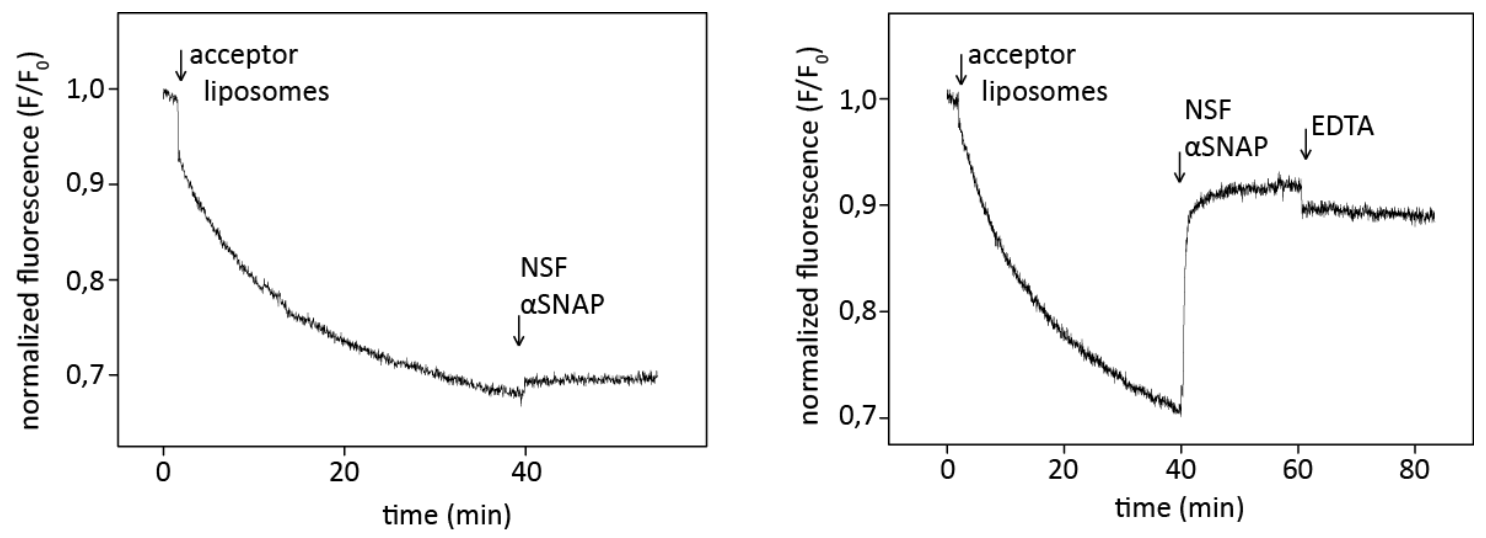

Figure 3.15. SNARE monomers do not re-assemble after trans complex disassembly. The experiment shown with the green trace in the right panel of figure 3.14 was repeated with the following changes. Left, the disassembly buffer was supplemented with EDTA (10 mM, total volume $600 \mu \mathrm{L})$. Right, EDTA $(10 \mathrm{mM})$ was added to the reaction 20 minutes after NSF and $\alpha$-SNAP additions. Fluorescence of the donor (Oregon Green labeled synaptobrevin) was normalized to its initial value $\left(F / F_{0}\right)$.

\subsubsection{NSF/ $\alpha$-SNAP-mediated disassembly of the acceptor complexes}

One explanation for no re-assembly would have been the fact that the acceptor sites were no longer available for synaptobrevin binding after NSF-driven disassembly. The acceptor complexes would present a binding site for $\alpha$-SNAP and get disassembled by NSF as well. Using an anisotropy assay, it was shown here that this was indeed the case. Anisotropy of the Texas Red label on SNAP-25 (SN25 TR) of the acceptor liposomes was used as a probe to monitor the disassembly reaction (see figure 3.16, left). Addition of $\alpha$-SNAP increased SN25 TR anisotropy (A), which indicated the binding of $\alpha$-SNAP to the acceptor complexes. And addition of NSF decreased SN25 TR anisotropy to a level that is lower than the initial anisotropy $\left(A_{0}\right)$. Considering that most of the complexes get reconstituted on the outer surface of the liposomes [149], this observation suggested that SN25 TR dissociated off the membrane upon disassembly. To test this possibility, trans complex disassembly reaction mix was run over a discontinuous Nycodenz gradient and the bottom 
fractions were checked for the presence of soluble SN25 TR (see figure 3.16, right). The fractions of the gradient were run on a Tricin SDS-PAGE gel which was analyzed by immunoblotting for the presence of SN25 TR. Since the bottom fractions contained SN25 TR, it was concluded that the labeled protein became soluble after trans complex disassembly. A control reaction was prepared without NSF and $\alpha$-SNAP where no disassembly occurred, and SN25 was found only in the top fractions associated with the liposomes. Since the SN25 antibody (Cl. 71.1, SySy) was reported earlier not to recognize SN25 in ternary complexes, the bands running above the SN25 band might correspond to the SN25 homo-oligomers [47].
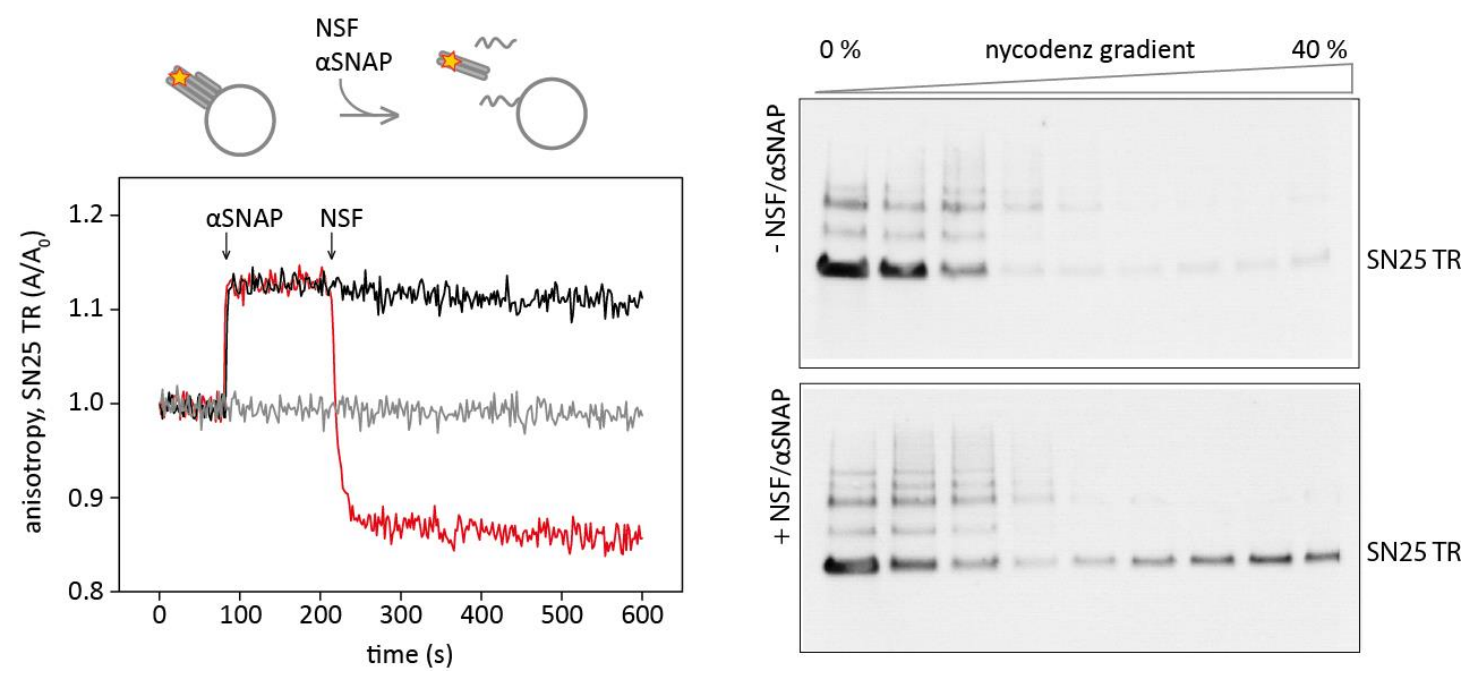

Figure 3.16. SN25 dissociates off the membrane upon disassembly. Left, Texas Red labeled acceptor complexes were reconstituted on large liposomes. In disassembly buffer (total volume $600 \mu \mathrm{L})$, acceptor liposomes $(5 \mu \mathrm{L})$ were mixed with $\alpha$-SNAP $(1 \mu \mathrm{M})$ and NSF $(60 \mathrm{nM})$, sequentially. Anisotropy of SN25 TR (A) was monitored and normalized to its initial anisotropy $\left(A_{0}\right)$. TR label of SN25 is shown with a red star. On the cartoon schematics, after the disassembly step, the soluble proteins SN25 TR and syb (49-96) are shown in solution whereas the membrane protein syntaxin $(183-288)$ is shown on the membrane. Right, the experiment shown with the red trace in the right panel of figure 3.12 was repeated in the presence (bottom panel) or absence (top panel) of NSF/ $\alpha$-SNAP. The reaction was then analyzed on a discontinuous Nycodenz gradient and the fractions after ultracentrifugation were tested for SN25 TR using immunoblotting a monoclonal SNAP-25 antibody (Cl. 71.1, SySy). 
Results

\subsubsection{Trans SNARE complexes are not "NSF-resistant"}

The investigations in this reconstitution system showed that upon NSF-mediated disassembly of the trans complexes, SNARE monomers do not re-assemble, acceptor complexes are taken apart and SN25 TR dissociates off the membrane. Yet, it remained unclear whether some of the trans complexes were resistant to NSF/ $\alpha$ SNAP. Such "NSF-resistant" complexes might have been contributing to the FRET signal after NSF/ $\alpha$-SNAP addition (see figure 3.12 and figure 3.14, right panels). To address this possibility, the susceptibility of synaptobrevin to cleavage by tetanus neurotoxin light chain (TeNT, kindly provided by Dr. Matias Hernandez) was examined. This toxin is known to cleave synaptobrevin only when it is not part of a SNARE complex (at a C-terminal position Q76-F77, [24, 46]). It recognizes an Nterminal region of synaptobrevin, which becomes hidden upon SNARE complex assembly [109]. This is essentially the reason why the toxin cannot process the synaptobrevin in a SNARE complex. Thus, TeNT would serve as a valuable tool to test if syb $\Delta 84$ monomer was found as a free monomer or in an NSF-resistant trans complex.

Unlabeled acceptor liposomes were mixed with Oregon Green labeled syb $\Delta 84$ (syb $\triangle 84$ OG) liposomes to generate single labeled trans SNARE complexes between docked liposomes. The liposomes were then incubated sequentially with NSF/ $\alpha$ SNAP and TeNT. Cleavage resulted in a shorter cytoplasmic fragment that was separated from the uncleaved protein by Tricin -SDS-PAGE with both being visible due to the fluorescent label at position 28.

Figure 3.17 shows four reactions that are depicted with the letters a-d. In reactions c and $d$, single labeled trans complexes assembled in the absence of NSF/ $\alpha$-SNAP. Syb $\triangle 84$ OG in these complexes was not cleaved by TeNT, implicating that the TeNT recognition site is buried in the trans complex. However, in the presence of the disassembly machinery about half of the syb $\Delta 84$ OG fluorescence was obtained from cleaved protein (reaction b). It was reported earlier that only half of the synaptobrevin monomers get reconstituted with their cytoplasmic regions on the 
outer surface of the large liposomes [149]. This was verified by incubating the syb $\Delta 84$ OG liposomes alone with NSF/ $\alpha$-SNAP and TeNT (reaction a). When all syb $\Delta 84$ OG on the outer surface of the liposomes were cleaved, this corresponded to about $50 \%$ of the fluorescence signal.

Taken together, this assay demonstrated that on the outer surface of the docking liposomes, almost all syb $\Delta 84$ OG assemble into TeNT-resistant trans SNARE complexes which are all disassembled when mixed with NSF and its co-factor.
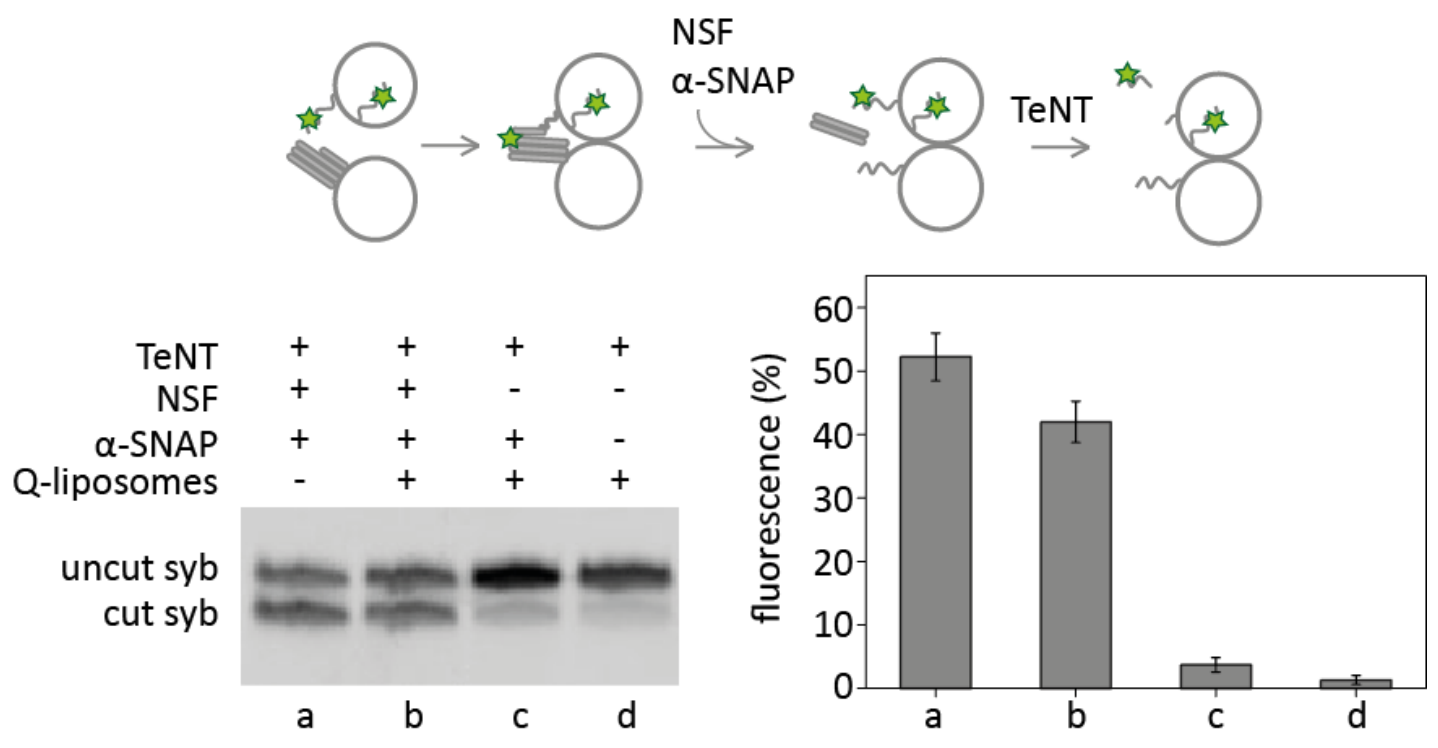

Figure 3.17. Almost all of the syb $\Delta 84$ monomers assemble into trans SNARE complexes which are not NSF-resistant. Liposome cartoons on the top depict the schematic steps of the second reaction (b). Unlabeled acceptor liposomes $(10 \mu \mathrm{L})$ were mixed with Oregon Green labeled syb $\Delta 84$ liposomes $(10 \mu \mathrm{L})$ for $30 \mathrm{~min}$ in disassembly buffer (total volume $100 \mu \mathrm{L})$. In the following step, $\alpha$-SNAP $(1 \mu \mathrm{M})$ and NSF (60 nM) were added. After 10 minutes of disassembly incubation, TeNT $(1 \mu \mathrm{M})$ was added and incubated for another 10 minutes. The Oregon Green label on syb $\Delta 84$ was depicted with a green star. This reaction was repeated leaving out the followings: unlabeled acceptor liposomes (a), NSF (c), NSF and $\alpha$-SNAP (d). All four reactions were analyzed via Tricin-SDS-PAGE gels and scanned for the Oregon Green fluorescence on syb $\Delta 84$ (left panel). These reactions were repeated with different liposome preparations on a different day (data not shown). The relative intensities of the fluorescence bands which correspond to the cut syb $\Delta 84$ monomers (the lower bands in the lanes from a-to-d) were calculated for these two different sets of experiments. ImageJ software was used to do the calculations and the fluorescence (\%) is presented via a histogram plot (right panel). Error bars represent standard deviation. 
Results

TeNT was also incorporated to the FRET-based bulk assay and trans complex assembly/disassembly cycle was monitored (see figure 3.18, top). Addition of TeNT either before or after NSF/ $\alpha$-SNAP to the reaction, recovered the donor fluorescence fully. Repeating the bulk assays using an inactive TeNT mutant, did not reveal such an effect (data not shown, [158]). It was possible that Oregon Green labeled syb $\Delta 84$ might be also found in solution upon trans complex disassembly and TeNT activity. Essentially, such a state might correspond to a similar chemical environment of the Texas Red and Oregon Green labeled SNARE monomers of the disassembled cis complexes and explain the full recovery of the donor fluorescence (see figure 3.12, left).

The reaction mix prepared for the FRET-based bulk assay with TeNT was then studied at a single-molecule level using fluorescence lifetime analysis (see figure 3.18, bottom). Excited state lifetime of the donor (syb $\Delta 84$ OG) was reduced upon mixing the acceptor and syb $\Delta 84$ liposomes, as trans complexes formed. Addition of NSF/ $\alpha-$ SNAP enhanced the donor lifetime significantly. This observation provided another line of evidence on trans SNARE complex disassembly. Donor lifetime remained unchanged in the absence of $\mathrm{MgCl}_{2}$ (data not shown). Addition of TeNT recovered the donor lifetime fully confirming the conclusions derived from the FRET-based bulk assay. 

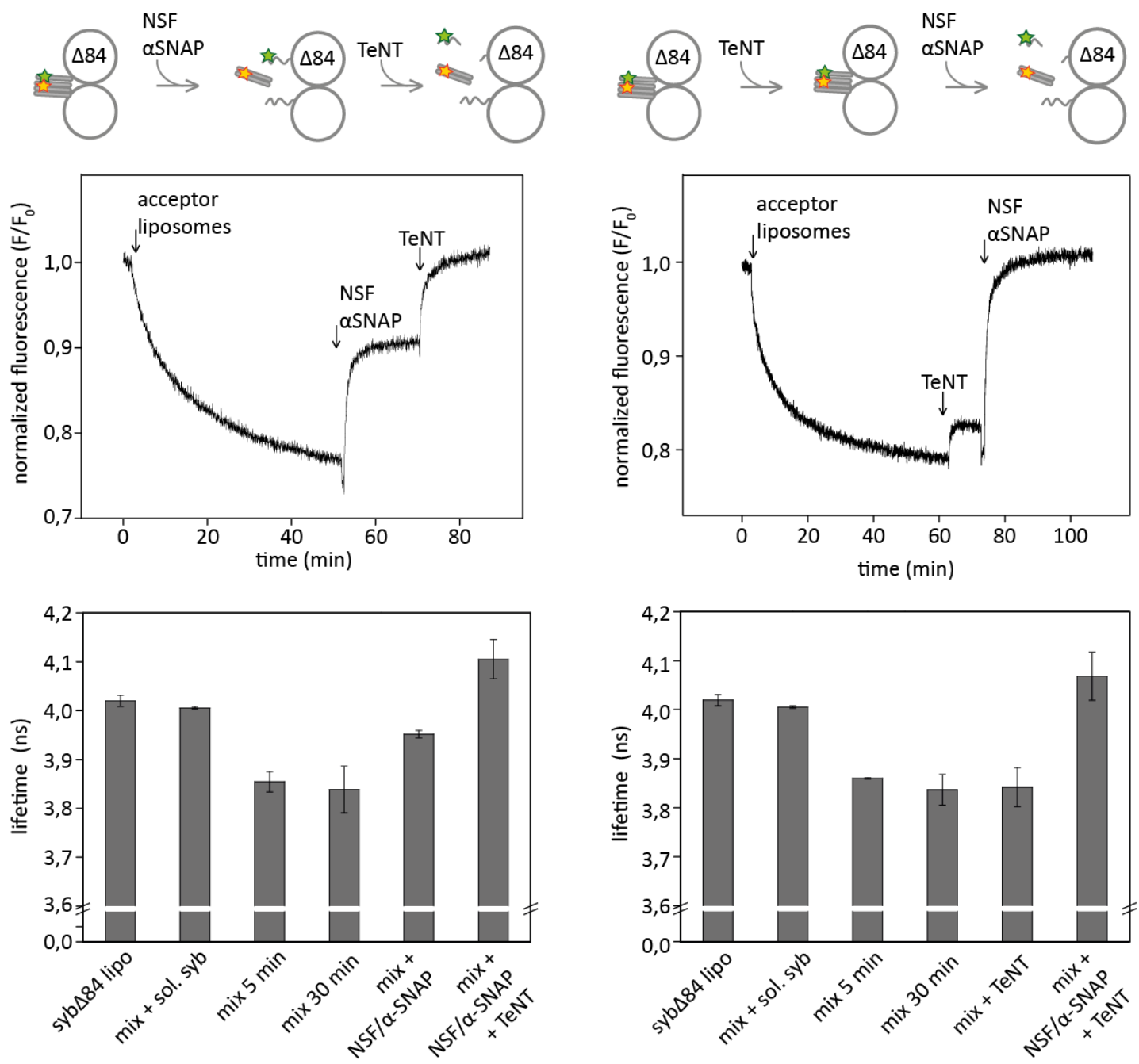

Figure 3.18. Incorporating TeNT to the NSF-driven trans complex disassembly reaction recovered Oregon Green labeled syb $\Delta 84$ fluorescence fully. Top panel, FRET-based bulk assay was performed as in figure 3.12. Following the NSF-mediated disassembly, TeNT (1 $\mu \mathrm{M}$ ) was added to the reaction (left). This reaction was repeated by altering the order of NSF/ $\alpha$-SNAP and TeNT additions (right). Fluorescence of the donor (Oregon Green labeled syb $\Delta 84)$ was normalized to its initial value $\left(F / F_{0}\right)$. Bottom panel, for fluorescence lifetime analyses the same liposomes were mixed shaking at $37^{\circ} \mathrm{C}$ for 30 minutes (in $300 \mu \mathrm{L}$ disassembly buffer). NSF/ $\alpha$-SNAP and TeNT were added sequentially, each for 10 minutes incubations (left). This reaction was repeated by altering the order of NSF/ $\alpha$-SNAP and TeNT additions (right). $10 \mu \mathrm{L}$ samples were taken from the reactions at $0,5,30$ minutes and after NSF, TeNT incubations. Fluorescence lifetime of Oregon Green labeled syb $\Delta 84$ was measured for $10 \times 15 \mathrm{~s}$ (out of three sets of mixes). Excess soluble syb wt (1-96) was added to the negative control reactions which were depicted as ' $\mathrm{mix}+$ sol. syb'. Lifetime of syb $\Delta 84$ liposomes alone was also measured and were depicted as 'syb $\Delta 84$ lipo'. Error bars represent standard deviation. 
Results

\subsection{Trans SNARE complex dynamics: loosely zippering complexes}

Studies with syb $\Delta 84$ suggested that a prerequisite to study partially assembled complexes in vitro was to arrest such complexes between docked large proteoliposomes. In this state, zippering of the SNARE monomers halts and prevents liposome fusion. This section presents the studies with which synaptobrevin double substitution mutant, syb AA (see section 1.2.1 and 1.3) was tested for a similar docking phenotype that syb $\Delta 84$ exhibits.

First, the interactions between large syb AA liposomes and large acceptor liposomes were studied. For this, NBD/Rho-labeled acceptor liposomes were mixed either with unlabeled syb wt liposomes or with unlabeled syb AA liposomes. NBD fluorescence was monitored for lipid mixing (see figure 3.19). The increase of fluorescence (NBD dequenching) indicated the fusion of acceptor liposomes with syb wt liposomes. When excess amounts of soluble syb wt (1-96) were included, they "silenced" the syb wt binding site of the acceptor complexes and hence no liposome fusion occurred. Interestingly, mixing acceptor liposomes with syb AA liposomes did not yield any increase in NBD fluorescence, demonstrating that these liposomes did not fuse. In fact, NBD fluorescence of the syb AA reaction overlapped with that of the control reaction with excess soluble syb wt (1-96).

The fact that large syb AA liposomes did not fuse with the large acceptor liposomes might have had two possible explanations. The acceptor complexes and syb AA mutant might have not interacted, and hence large liposomes might have failed to dock in a SNARE-dependent manner. Alternatively, SNARE interaction and liposome docking might have occurred, but zippering might have stalled before the synaptobrevin and syntaxin linker regions and inhibited liposome fusion. Fluorescence cross-correlation spectroscopy (FCCS) experiments revealed that the latter was the indeed the case (see section 3.4). The extent of liposome docking was very similar when acceptor liposomes were mixed either with syb $\Delta 84$ liposomes or with syb AA liposomes. It seemed that the acceptor liposomes docked with syb AA liposomes and failed to fuse. 


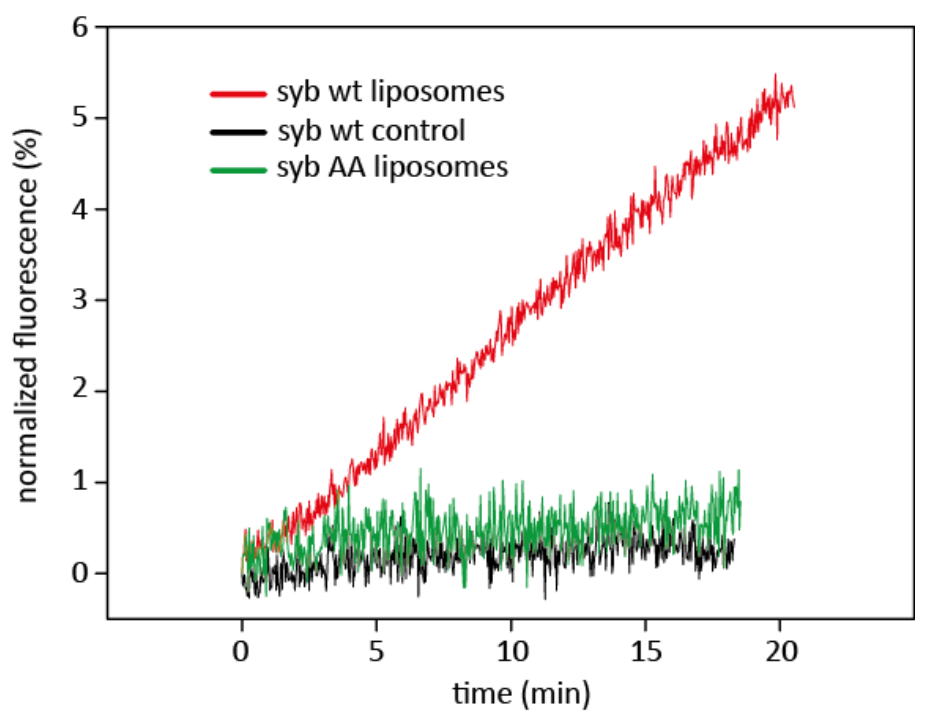

Figure 3.19. Large syb AA liposomes do not fuse with large acceptor liposomes. Acceptor complexes were purified from full length syx (1-288), SN25 (1-206), syb (49-96) and reconstituted on NBD/Rho-labeled large liposomes. Acceptor liposomes (15 $\mu \mathrm{L})$ were then mixed with unlabeled syb wt liposomes or with unlabeled syb AA liposomes $(15 \mu \mathrm{L})$ in liposome buffer (total volume $1,2 \mathrm{~mL}$ ) at $30^{\circ} \mathrm{C}$. NBD fluorescence was monitored and normalized to the maximum fluorescence as explained earlier (in 2.2.4.2).

Together, the data described above suggest that the syb AA mutant leads to a fusionarrest in an only partially zippered state, in contrast to the syb $\Delta 84$ mutant where fusion is also arrested but the SNARE motifs are fully zippered. In the following set of experiments, the nature of syb AA trans complexes were studied by comparing them with syb $\Delta 84$ trans complexes.

First, the established FRET pair (see figure 3.11 and figure 3.12) was used to investigate the protein-protein interactions between the acceptor complexes and syb AA mutant. The labeling position of syb AA (28 ${ }^{\text {th }}$ cysteine residue) is upstream of the double alanine substitutions ( $45^{\text {th }}$ and $46^{\text {th }}$ alanine residues). Even if there was an arrest at around the $-3^{\text {rd }}$ layer, the zippering up to this region would still yield a FRET signal. For this, two sets of large liposomes were reconstituted with Texas Red labeled acceptor complexes and Oregon Green labeled syb AA, respectively. Fluorescence emission of the donor was monitored as acceptor liposomes were mixed with syb AA liposomes. In a reference reaction, the assembly of syb $\Delta 84$ trans complexes was monitored. As shown in figure 3.20, trans SNARE complex assembly reactions of syb 
Results

$\Delta 84$ and syb AA exhibited a marked difference. Fluorescence emission of syb AA indicated that there was no fast zippering until the double alanine block. This might have occurred due to two possible reasons. Either the liposome docking was very slow or the zippering was arrested upstream of the double alanine block for an inexplicable reason. FCCS experiments (see figure 3.26) crossed out the first possibility demonstrating similar docking rates for both syb AA liposomes and for syb $\Delta 84$ liposomes. However, the nature of the zippering syb AA trans complexes remained inconclusive. The subsequent anisotropy experiments emphasized the difference in the extent of zippering before the $-3^{\text {rd }}$ layer among the two synaptobrevin mutants.
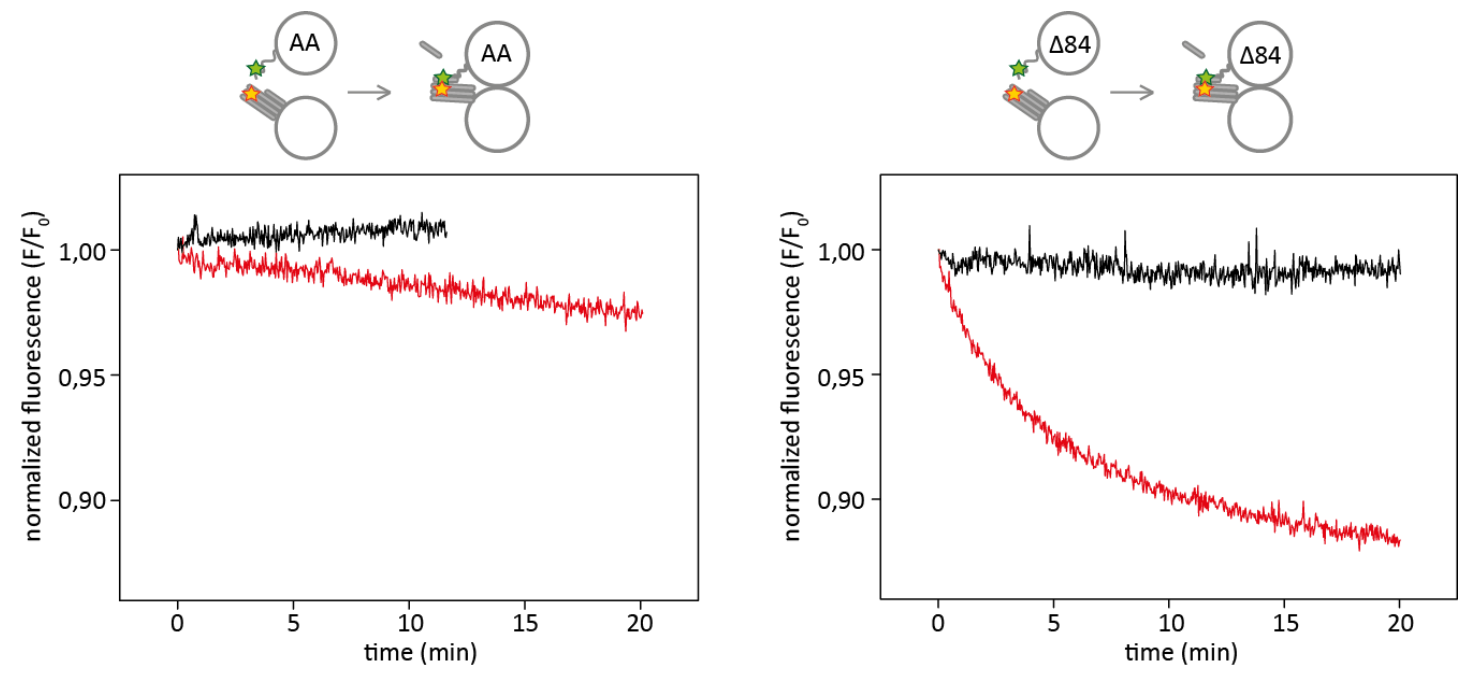

Figure 3.20. Trans SNARE complex assembly reactions of the two synaptobrevin mutants are monitored via the FRET-based bulk assay. Texas Red labeled acceptor liposomes $(5 \mu \mathrm{L})$ were mixed either with Oregon Green labeled syb AA liposomes $(5 \mu \mathrm{L}$, left) or with Oregon Green labeled syb $\Delta 84$ liposomes ( $5 \mu \mathrm{L}$, right). The reactions were performed in disassembly buffer with (black) or without (red) excess amounts of unlabeled syb wt (1-96). Fluorescence emission (F) of the donor (Oregon Green labeled synaptobrevin) was monitored and normalized to the initial fluorescence ( $\left.F_{0}\right)$. Red stars and green stars depict Texas Red label on SNAP-25 and Oregon Green label on synaptobrevin, respectively. The rod cartoon represents the short syb (49-96) fragment of the acceptor complexes which is displaced off the membrane upon complex formation.

To address the displacement of the syb (49-96) fragment, an acceptor complex was purified from the following monomers, syx (183-288), SN25 (1-206) and Oregon Green labeled syb (49-96). Large liposomes were reconstituted with this acceptor 
complex and were mixed with different unlabeled synaptobrevin liposomes. Displacement of the labeled syb (49-96) by synaptobrevin monomers (syb wt, syb $\Delta 84$ and syb AA) were compared via fluorescence anisotropy (see figure 3.21).

Before, it had been shown that syb (49-96) was displaced off the acceptor complex liposomes at similar rates by syb wt and syb $\Delta 84$ [38]. As expected, in this assay, the anisotropy of syb (49-96) decreased very similarly upon the additions of syb wt or syb $\Delta 84$ liposomes. Interestingly, as acceptor liposomes were mixed with syb AA liposomes, the anisotropy did not reveal a similar change.

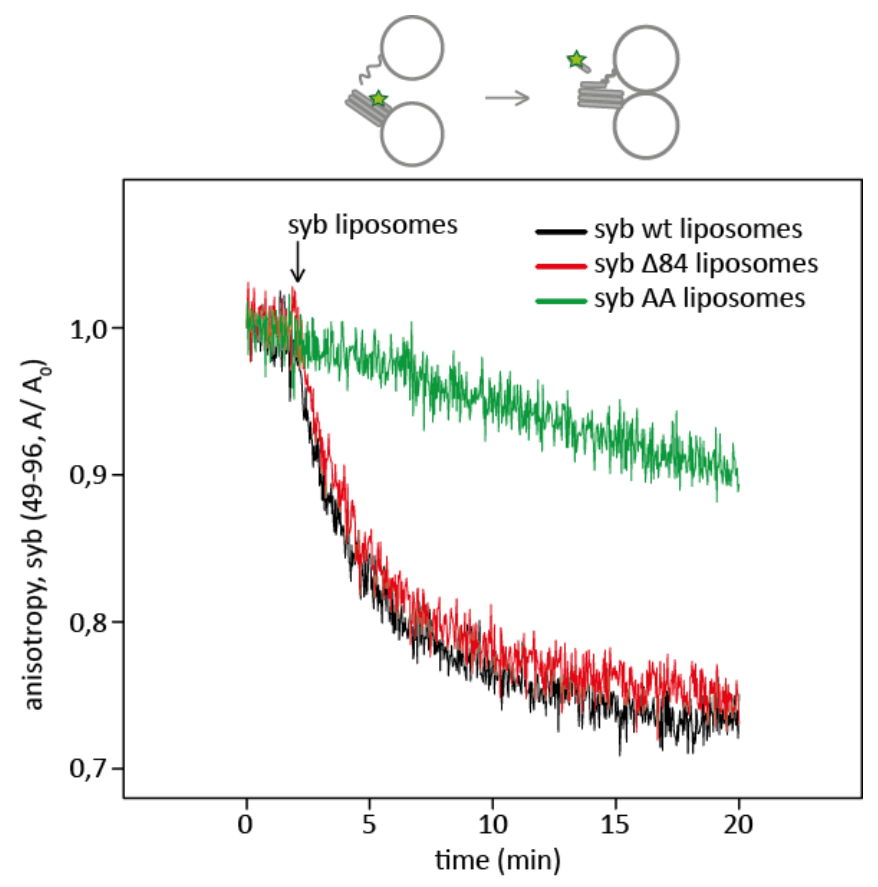

Figure 3.21. Displacement of Oregon Green labeled syb (49-96) by two synaptobrevin mutants are compared via fluorescence anisotropy. The acceptor complexes were labeled at the T79C residue of syb (49-96) fragment (green star cartoon) and were reconstituted on large liposomes. Acceptor liposomes $(12 \mu \mathrm{L})$ were mixed with different synaptobrevin liposomes (12 $\mu \mathrm{L}$, unlabeled liposome cartoon) in liposome buffer (total volume $600 \mu \mathrm{L}$ ). The anisotropy (A) of the Oregon Green labeled syb (49-96) fragment was monitored and normalized to its initial value $\left(A_{0}\right)$.

It was not clear whether the anisotropy read-out using the syb AA liposomes indicated a very slow syb (49-96) displacement or a non-specific anisotropy signal. For this reason, an additional fluorescence anisotropy assay was developed to monitor the binding of synaptobrevin to the acceptor complexes on large liposomes. 
Results

In this assay, the anisotropy of Oregon Green labeled syb $\Delta 84$ and syb AA monomers on liposomes were monitored. Unlabeled acceptor liposomes were mixed with either of the synaptobrevin liposomes.

Anisotropy of the syb $\Delta 84$ monomer on large liposomes increased as it was binding to the acceptor liposomes (figure 3.22, left). This increase was inhibited when excess amounts of unlabeled soluble syb wt (1-96) was included in the reaction and "silenced" the acceptor sites for the labeled syb $\Delta 84$. Unlabeled acceptor liposomes were mixed with Oregon Green labeled syb wt liposomes as well (data not shown). When normalized anisotropies of syb wt and syb $\Delta 84$ were overlaid, their signals overlapped. This trend confirmed the previous syb (49-96) displacement experiments (see figure 3.21, black and red traces). Finally, unlabeled liposomes were mixed with Oregon Green labeled syb AA liposomes (see figure 3.22, right). However, the anisotropy of syb AA did not change upon mixing. It was even overlapping with the control reaction with excess unlabeled soluble syb wt (1-96).

The bulk FRET and the fluorescence anisotropy experiments did not provide any evidence for a complex formation between the syb AA mutant and the acceptor complex. The anisotropy signals were more conclusive since they reported on the impairments on the rotational diffusion of the labeled proteins and were not dependent on the Forster distances. Even if there were SNARE interactions involving the syb AA mutant, it was not possible to monitor these interactions with the $28^{\text {th }}$ labeling position. Therefore, the last set of experiments of this section took the advantage of the TeNT cleavage, which does not attack assembled complexes. These experiments checked for the existence of TeNT-resistant syb AA trans complexes. 

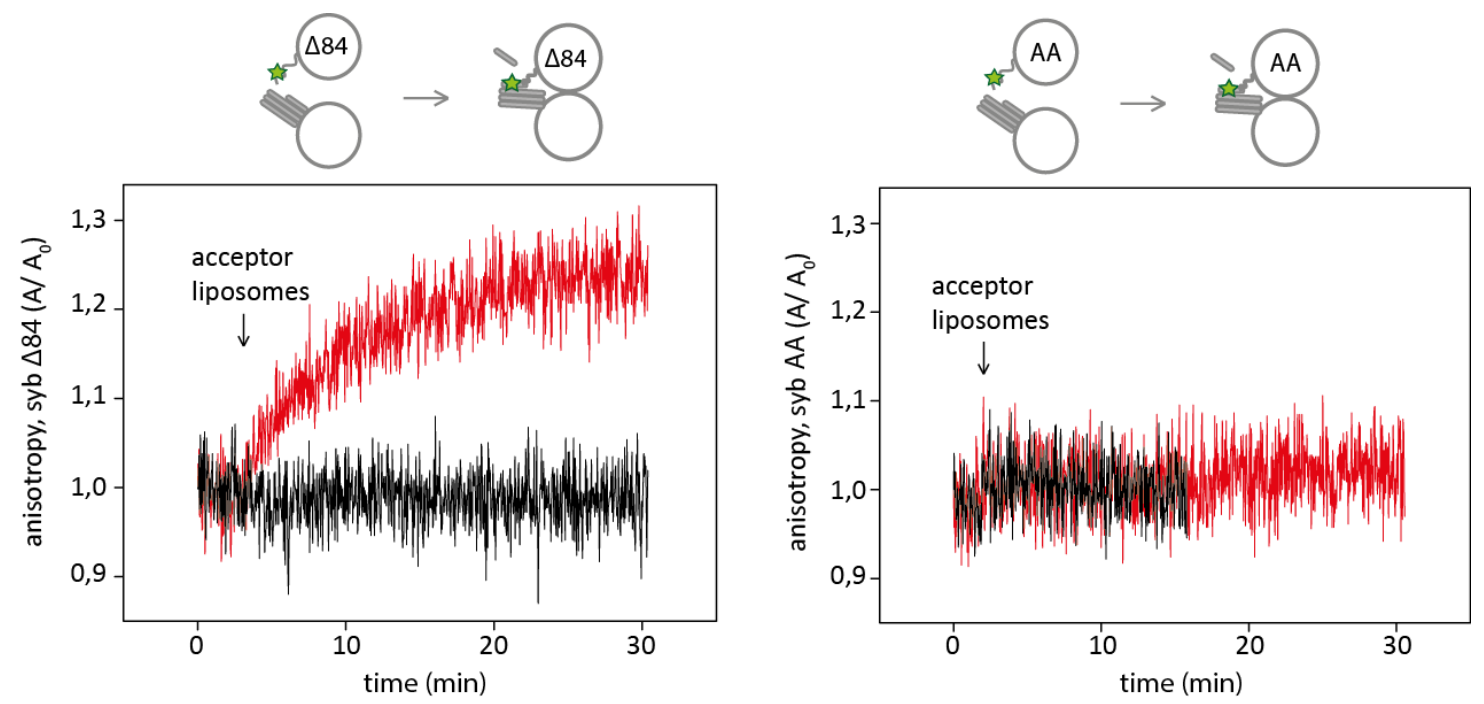

Figure 3.22. Trans SNARE complex assembly reactions of the two synaptobrevin mutants are monitored via fluorescence anisotropy. Monomers of syb $\Delta 84$ and syb AA were labeled with Oregon Green at their $28^{\text {th }}$ residues and were reconstituted on large liposomes. Unlabeled acceptor liposomes $(5 \mu \mathrm{L})$ were mixed either with syb $\Delta 84$ liposomes ( $5 \mu \mathrm{L}$, left) or with syb AA liposomes $(5 \mu \mathrm{L}$, right). The anisotropy $(A)$ of Oregon Green labeled synaptobrevin on liposomes was monitored and normalized to its initial value $\left(A_{0}\right)$. Red traces represent the reactions in disassembly buffer (total volume $600 \mu \mathrm{L}$ ), whereas the black traces represent the control reactions repeated with excess (75-fold) unlabeled syb wt (1-96).

The gel-based fluorescence assay (see section 3.2.5) was performed, this time using syb AA liposomes. Here, unlabeled acceptor liposomes were mixed with Oregon Green labeled syb AA liposomes. NSF/ $\alpha$-SNAP and TeNT were added sequentially to the reaction. Lastly, syb AA fluorescence was analyzed via Tricin-SDS-PAGE.

Figure 3.23 shows four reactions that are depicted with the letters a-d. In reactions $c$ and $d$, the acceptor liposomes were mixed with syb AA liposomes in the absence of NSF and $\alpha$-SNAP. Remarkably, some of syb AA OG on the outer surface of the liposomes was not cleaved by TeNT. This observation provided strong evidence for a TeNT-resistant trans interaction involving the syb AA mutant, which is clearly different from syb $\Delta 84$ trans complexes. Furthermore, with the reaction $\mathrm{c}$, these TeNT-resistant loose trans complexes were shown to be disassembled by NSF and $\alpha$ SNAP. 
Results
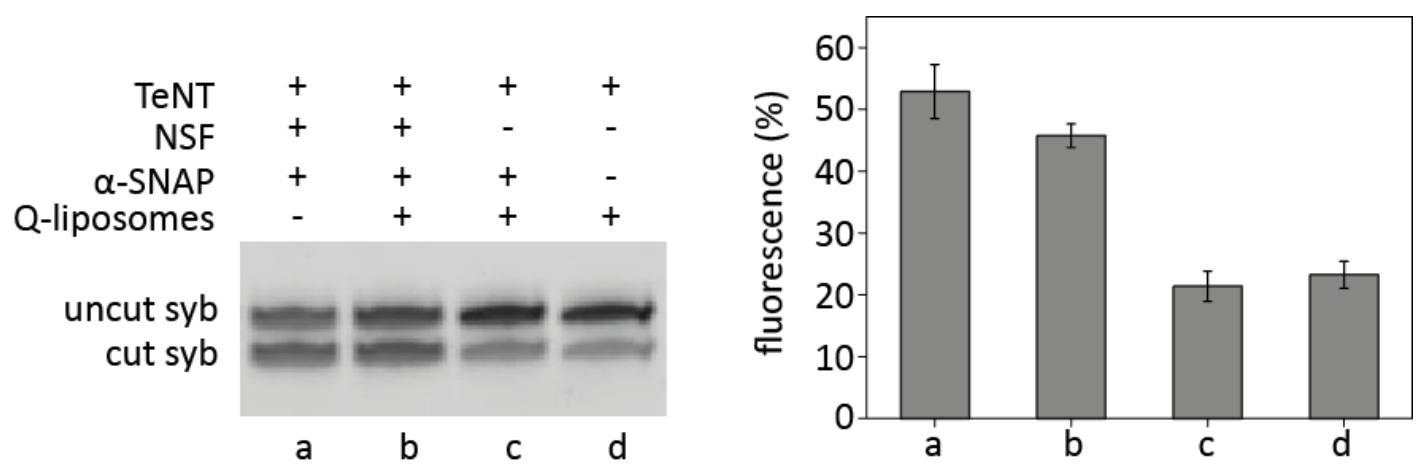

Figure 3.23. Some of the syb AA monomers assemble into trans SNARE complexes which are TeNT-resistant. Unlabeled acceptor liposomes $(10 \mu \mathrm{L})$ were mixed with Oregon Green labeled syb AA liposomes $(10 \mu \mathrm{L})$ for 30 min in disassembly buffer (total volume $100 \mu \mathrm{L}$ ). In the following step, $\alpha$-SNAP $(1 \mu \mathrm{M})$ and NSF (60 $\mathrm{nM})$ were added. After 10 minutes of disassembly incubation, TeNT $(1 \mu \mathrm{M})$ was added and incubated for another 10 minutes. This reaction was depicted with the letter ' $c$ ' and repeated leaving out the followings: unlabeled acceptor liposomes (a), NSF (c), NSF and $\alpha$-SNAP (d). All four reactions were analyzed via Tricin-SDS-PAGE gels and scanned for syb AA fluorescence (left panel). These reactions were repeated with a second liposome preparation on a different day (data not shown). The relative intensity of the fluorescence bands which corresponded to the cut syb AA (lower bands) were calculated for the two sets of liposomes. ImageJ software was used for the calculations and the fluorescence (\%) were represented here via a histogram plot (right panel). Error bars represent standard deviation.

\subsection{Membrane dynamics}

Previous two sections presented the fluorescence spectroscopic bulk assays that monitored the dynamics of the trans SNARE complexes. These assays reported that the deletion mutant syb $\Delta 84$ associated with the acceptor complexes to form tightly zippered trans complexes on large liposomes that did not fuse confirming the recent study of this mutant [38]. Interestingly, NSF was capable of fully disassembling these tight trans complexes demonstrating that they were not protected as it was claimed earlier [133] and answering one of the questions that this study aimed to address. Syb AA large liposomes were also found not to be fusing. Intriguingly, there was no evidence for partial zippering of the syb AA mutant's SNARE motif (neither via FRET nor via fluorescence anisotropy). Nevertheless, interactions of this mutant with the acceptor complexes resulted in partial TeNT resistance, suggesting an arrest in zippering at the very early stage of these interactions. Therefore, it was speculated 
that the trans SNARE complexes of the syb AA mutant had a very different structure from that of the syb $\Delta 84$ mutant.

Bulk fluorescence spectroscopic assays used fluorescently labeled protein probes. The final section of this chapter presents the docking/undocking of the liposomes using fluorescently labeled lipid probes. Fluorescently labeled large liposomes were reconstituted with unlabeled acceptor complexes and synaptobrevin layer mutants, respectively. Fluorescently labeled lipids were monitored to study the interactions of the liposomes as SNAREs assembles into partial complexes between them. The first set of experiments (see section 3.4.1) investigated whether NSF-mediated disassembly of the trans complexes reverted the docking liposomes to their previous undocking state. And the following section (see section 3.4.2) examined the trend of liposome docking while loose syb AA trans interactions occurred between the liposomes. It was questioned if such interactions were enough to dock syb AA liposomes to the acceptor liposomes.

\subsubsection{Do liposomes stop docking if all of the trans complexes are fully disassembled by NSF?}

Recently, a fluorescence cross correlation spectroscopy (FCCS) method has been developed to study the extent of SNARE-mediated liposome docking quantitatively $[154,155]$. This method measures the fluorescence fluctuations caused by the diffusion of the liposomes carrying Oregon Green or Texas Red labeled lipids in a twophoton excitation volume of a confocal microscope setup. The average particle number in this volume with both types of labeled lipids (cross-correlation) is compared with the total number of the particles with red or green labels (autocorrelation). This comparison essentially allows for quantification of the ratio of the docked liposomes and the total liposome population. One crucial point to determine this ratio as accurate as possible was to mix the green and red labeled liposomes in a 1:1 stoichiometry [154]. This was attained by testing the particle number for the red and green probes regularly. 
Results

With this method the extent of liposome docking mediated by the syb $\Delta 84$ trans complex formation was determined earlier [38]. It was found that almost all of the large liposomes were arrested in a tightly docked state with an extended contact zone (see the electron micrographs in [149]). Here, a similar FCCS approach was taken to answer whether these liposomes stop docking if all of the trans complexes were fully disassembled by NSF.

Texas Red labeled liposomes were reconstituted with the acceptor complex, whereas Oregon Green labeled liposomes were reconstituted with syb $\Delta 84$. These liposomes were mixed with a 1:1 stoichiometry (determined via the particle number function with the confocal setup). After a 30-minute-incubation, NSF and $\alpha$-SNAP were added to initiate the disassembly of the trans SNARE complexes. The extent of docking for each step of the assembly/disassembly reaction was assessed by analyzing $10 \mu \mathrm{L}$ samples of the reaction mix. The degree of fluorescence cross-correlation was calculated as a direct measure for the proportion of docked vs. total number of liposomes (see figure 3.24). This analysis revealed that almost all of the large liposomes were docked in a SNARE-dependent manner, after mixing acceptor liposomes with syb $\Delta 84$ liposomes for 30 minutes (see figure 3.24, left and [38]). But, very surprisingly, these liposomes were still docked even after NSF was added to the reaction. In fact, the extent of docking was in the same range for the control reactions where NSF was inactive. Although the trans complexes in between the docked liposomes were disassembled, the liposomes stayed in the tightly docked state.

These experiments were repeated by changing the protein:lipid ratio of the labeled liposomes to 3000:1. With less number of proteins on the large liposomes, less numbers of trans SNARE complexes were expected to form [159] and perhaps resolve the docking liposomes more easier once disassembled. As expected, mixing acceptor liposomes with syb $\Delta 84$ liposomes revealed a lower docking percentage (see figure 3.24 , right). However, these liposomes with low numbers of complexes were still docking even after NSF was added. 


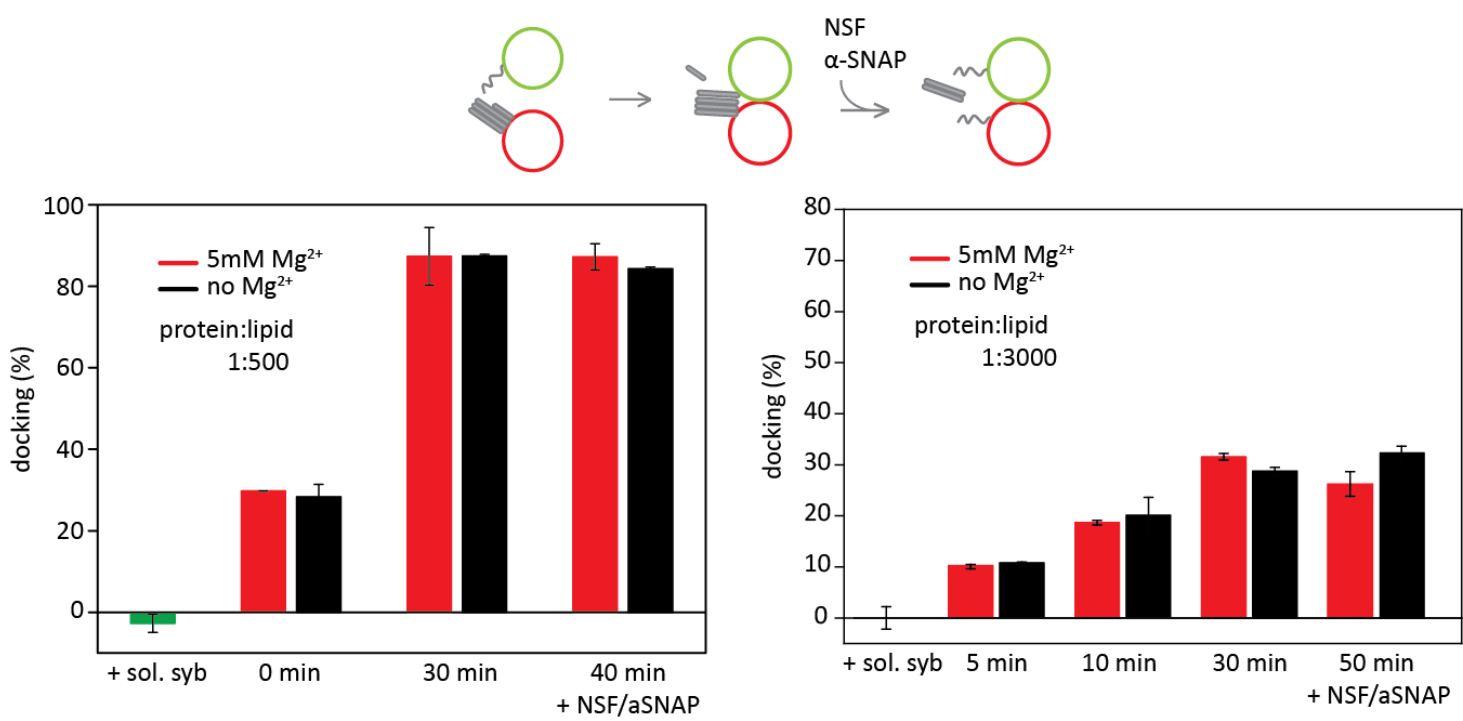

Figure 3.24. Large liposomes stay docked after trans complexes are disassembled. Fluorescently labeled lipids were included in lipid mixes of large liposomes. Texas RedDHPE labeled liposomes were reconstituted with the acceptor complexes, whereas Oregon Green-DHPE labeled liposomes were reconstituted with syb $\Delta 84$. Acceptor liposomes were mixed with synaptobrevin liposomes in disassembly buffer (total volume $300 \mu \mathrm{L}$ ) adjusted to obtain a 1:1 stoichiometry. They were incubated for 30 minutes in a thermo-shaker at $37^{\circ} \mathrm{C} .10 \mu \mathrm{L}$ samples were collected and analyzed at indicated time points. At the end of 30-min incubations, NSF $(60 \mathrm{nM})$ and $\alpha$-SNAP $(1 \mu \mathrm{M})$ were added and the final $10 \mu \mathrm{L}$ sample was collected. Control reactions for SNARE-dependent-docking were performed in the presence of excess soluble syb (1-96) and indicated with the green bar. Control reactions for NSF disassembly were performed in the absence of $\mathrm{MgCl}_{2}$ (black bars). Left, reactions performed with the liposomes with a protein:lipid ratio of 500:1 are shown. Right, reactions performed with the liposomes with a protein:lipid ratio of 3000:1 are shown. Each bar represents the average fluorescence cross correlation calculated for three independent reactions and error bars represent the standard deviation.

The next series of experiments brought a deeper understanding on the liposome docking phenomenon after NSF-mediated disassembly. These experiments were the repetitions of the previous FCCS experiments with slight changes. For this round, the duration of mixing was limited to shorter time windows and NSF/ $\alpha$-SNAP were added immediately after.

Recently, it was demonstrated that the trans SNARE complex formation starts from the moment of liposome mixing (see figure 3.21 and [38]). Keeping this in mind, here it was asked whether NSF can prevent SNARE-dependent liposome docking if introduced at the start of this SNARE zippering reaction. To test this, the acceptor 
Results

liposomes were mixed with syb $\Delta 84$ liposomes for shorter incubations when trans SNARE nucleation took place. At the end of these incubations, NSF was added immediately. The extent of liposome docking was measured once before and once after the disassembly took place.

Figure 3.25 shows three sets of experiments where Texas Red labeled liposomes were mixed with Oregon Green labeled liposomes for 0, 1 and 10 minutes. As it has been observed before, the extent of docking increased as the liposomes were given longer time to dock (see the time dependent increase in the black bars). When the measurement was taken at the time of mixing (0-minute-incubation), the docking percentage was around $20 \%$. Because each measurement lasted $10 \times 15$ seconds, liposomes had about 3 minutes to interact, which corresponded to $20 \%$ docking.

The comparison of the black bars with the red bars in these plots was a measure for the degree of docking before and after the disassembly reaction. Interestingly, NSF addition lowered the docking percentage only in the first seconds of the SNAREzippering. What had been observed for the liposomes that were incubated for 30 minutes (see figure 3.24), was again the case, even for the ones that were mixed only for 1 minute. If not present from the beginning of the docking, NSF-mediated disassembly did not affect the docking percentage. Matching results obtained as these experiments were repeated with a different set of liposomes on a different day (data not shown). Although NSF prevented docking for the 0 -minute-incubation reaction more strongly, it was not as effective in 1-minute-incubation reaction. 


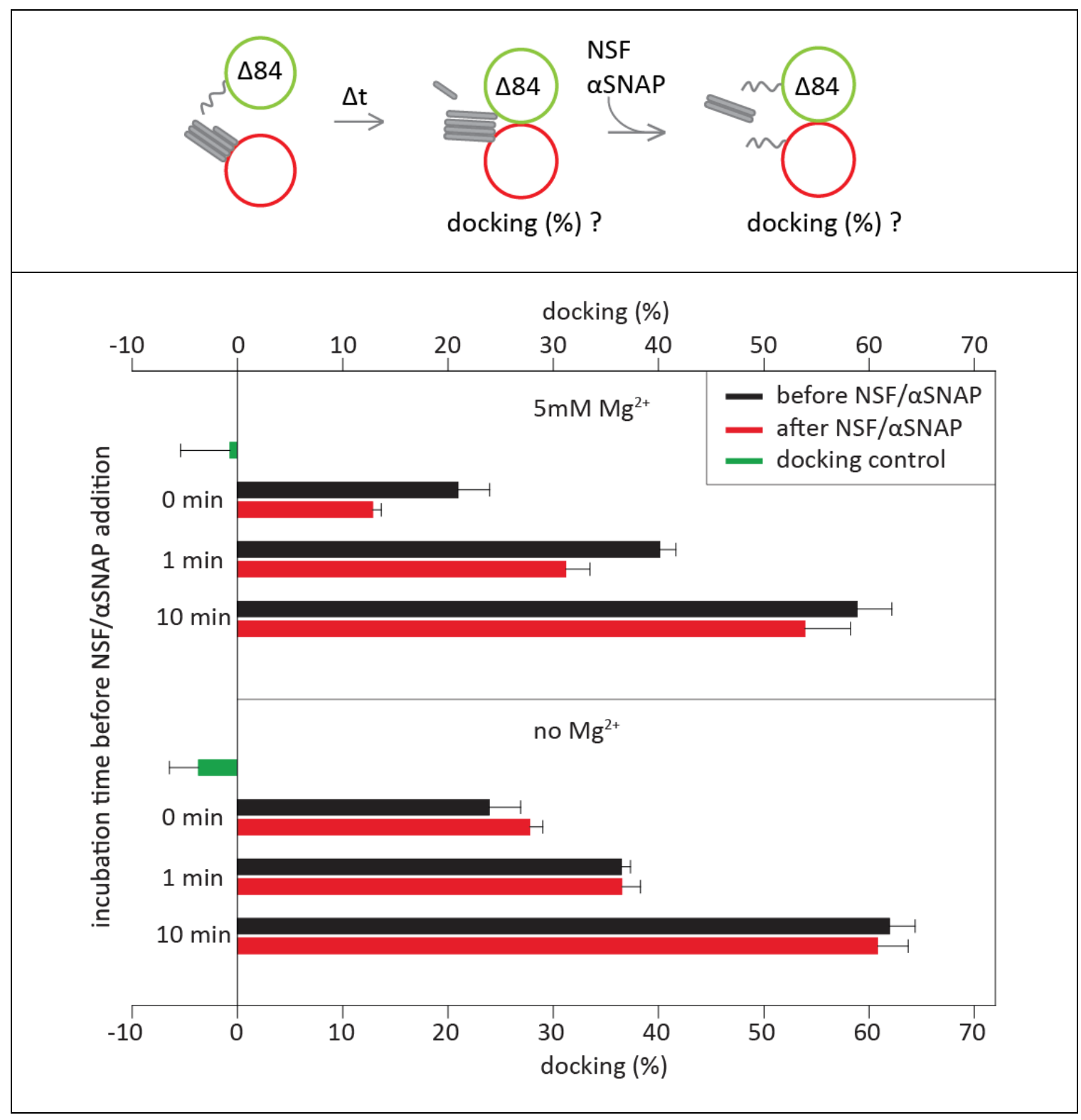

Figure 3.25. NSF/ $\alpha$-SNAP affects SNARE-dependent docking of large liposomes only at its initiation. Three different liposome mixes were prepared in the presence or absence of $5 \mathrm{mM} \mathrm{MgCl}$. TR-DHPE labeled acceptor liposomes were incubated with OG-DHPE labeled syb $\Delta 84$ liposomes (in 1:1 stoichiometry) for 0,1 and 10 minutes in disassembly buffer (total volume $300 \mu \mathrm{L}$ ) shaking at $37^{\circ} \mathrm{C}$. At the end of these incubations, NSF/ $\alpha$-SNAP were added to the reactions and incubated for 10 minutes. From each reaction, $10 \mu \mathrm{L}$ samples were collected before (black bars) and after (red bars) NSF addition and fluorescence cross correlation was analyzed to calculate the docking (\%). Control reactions for SNARE-dependent-docking were performed in the presence of excess soluble syb (1-96) and indicated with the green bars. Each bar represents the average fluorescence cross correlation calculated for three independent reactions and error bars represent the standard deviation. 
Results

FCCS experiments with short incubation times were repeated mixing Texas Red labeled acceptor liposomes with Oregon Green labeled syb AA liposomes (see figure 3.26). Remarkably, despite the failure to detect SNARE zippering via the FRET and fluorescence anisotropy assays, here the FCCS assays revealed that syb AA liposomes dock with the acceptor liposomes as efficiently as the syb $\Delta 84$ liposomes. Furthermore, the docking trend they exhibited was very similar to that of syb $\Delta 84$ liposomes. In 10 minutes almost half of the syb AA liposome population was docked to the acceptor liposomes. And as these 10-minute incubated liposomes were mixed with NSF and $\alpha$-SNAP, they continued docking as well like the syb $\Delta 84$ liposomes did. However, changing the liposome mix incubation time revealed profound differences in docking after the addition of NSF and $\alpha$-SNAP. In figure 3.26, the comparison of the gray bars with the blue bars revealed the docking percentages before and after the addition of NSF and $\alpha$-SNAP. In these experiments, NSF-mediated disassembly lowered the extent of docking substantially. The disassembly detached the liposomes that were incubated for 0 or 1 minutes prior to NSF addition. In addition, the docking percentages of the liposomes that were incubated even until 10 minutes were lowered after NSF-mediated disassembly, which was not the case in the previous experiments with syb $\Delta 84$ liposomes.

In the control experiments without $\mathrm{MgCl}_{2}$ shown with figure 3.25 and figure 3.26 , the same trends in docking were observed. The rate of docking did not change before and after NSF addition in these reactions. However, when NSF was not active in the absence of $\mathrm{MgCl}_{2}$, the rate of docking was expected to increase. Taking into account that $\alpha$-SNAP has recently been shown to have negative effects on SNARE complex zippering [143], the FCCS experiments were repeated in the absence of both $\mathrm{MgCl}_{2}$ and $\alpha$-SNAP (see Appendix A7). In these reactions, the rate of docking indeed increased in time. 


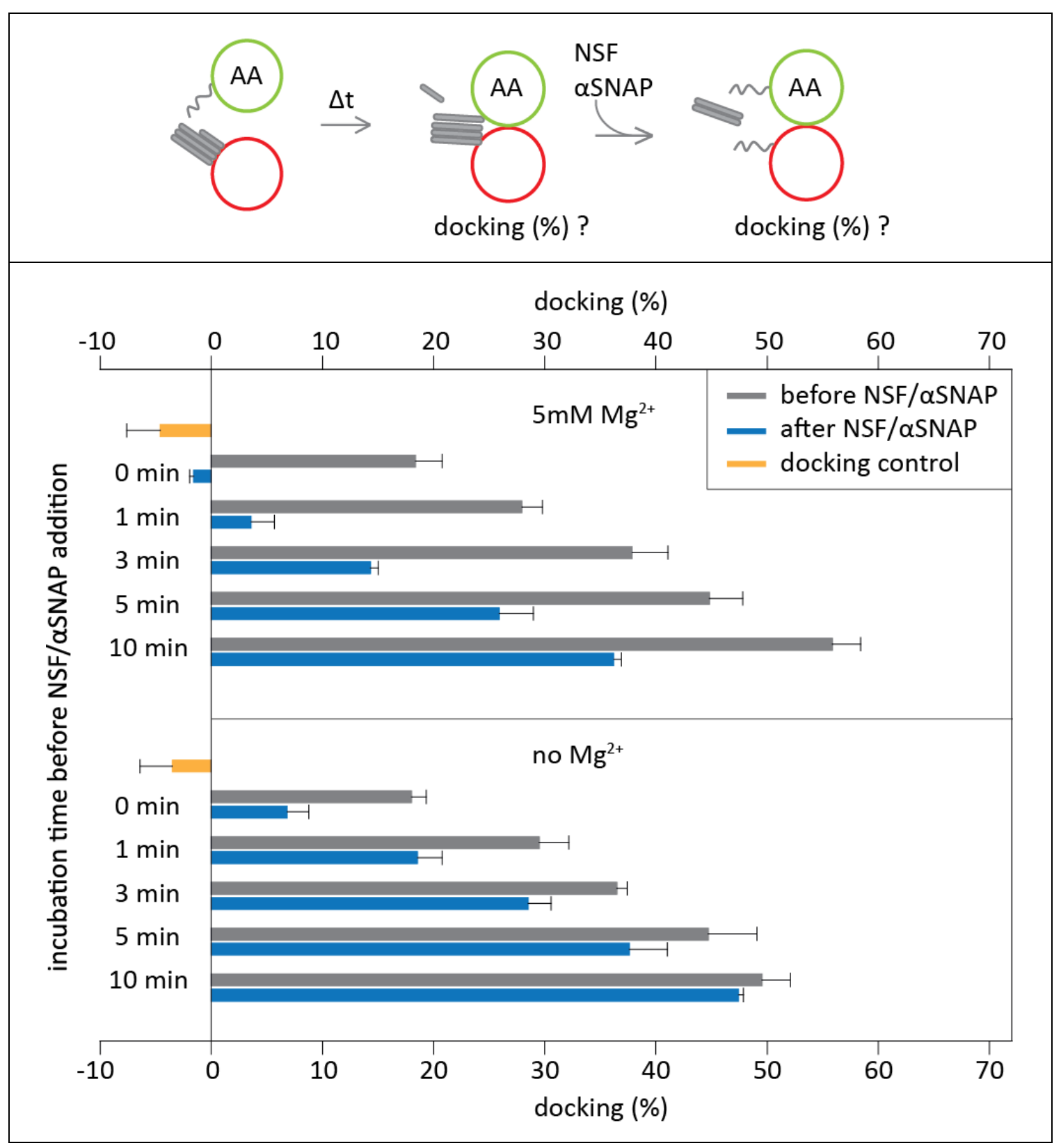

Figure 3.26. NSF/ $\alpha$-SNAP affects SNARE-dependent docking of large liposomes only at its initiation. The experiments shown with figure 3.25 were repeated using OG-DHPE labeled syb AA liposomes with extra incubation times for 3 and 5 minutes. At the end of these incubations, NSF/ $\alpha$-SNAP were added to the reactions and incubated for another 10 minutes. From each reaction, $10 \mu \mathrm{L}$ samples were collected before (gray bars) and after (blue bars) NSF addition and docking (\%) was analyzed as before. Orange bars indicate the control reactions for SNARE-dependent-docking, performed in the presence of excess soluble syb (1-96). Each bar represents the average fluorescence cross correlation calculated for three independent reactions and error bars represent the standard deviation. 
Results

\subsubsection{What is the extent of zippering in trans SNARE complexes?}

A second set of in vitro FCCS assays were developed to compare the SNARE zippering reactions for syb $\Delta 84$ and syb AA mutants. From the extent of SNARE-dependent liposome docking, it was possible to deduce the zippering states of trans SNARE complexes assembling between liposomes. In fact, these FCCS assays were developed upon the observations obtained via fluorescence anisotropy which are also described in this last section (see figure 3.27).

In the experiments that are shown with the previous three figures, liposome docking was caused by two reactions that operated sequentially. The first reaction was the trans SNARE complex nucleation. Once this nucleation had started liposomes came together and another reaction of irreversible docking took place. The extent of this second reaction also increased in a time-dependent manner. Moreover, irreversible docking was independent of the presence of SNARE complexes (see figure 3.24). In the light of these observations, following fluorescence anisotropy and FCCS assays were designed to address whether SNARE nucleation could be inhibited and/or reverted by soluble synaptobrevin (1-96) fragments (see figure 3.27) and whether such an inhibition would prevent the irreversible docking reaction (see figure 3.28).

For the fluorescence anisotropy experiments, the same acceptor complexes introduced in the experiments above (see figure 3.21) were used. Liposomes that were reconstituted with Oregon Green labeled acceptor complexes were mixed with unlabeled syb AA liposomes only for 3 minutes and an excess amount of unlabeled syb wt (1-96) was added to the reaction immediately after (see figure 3.27, left). Upon this addition, the anisotropy of Oregon Green labeled syb (49-96) decreased very rapidly, indicating that most of this fragment had been displaced off the acceptor complex liposomes. Acceptor liposomes were mixed with unlabeled syb $\Delta 84$ liposomes and unlabeled syb wt liposomes as well (see figure 3.27, right). In these reactions, after 3 minutes, addition of the unlabeled syb wt (1-96) decreased the anisotropy of the syb (49-96) fragment to a similar level. Mixing the acceptor liposomes directly with unlabeled excess syb wt (1-96) resulted in a rapid decrease 
of syb (49-96) anisotropy (see the black trace in figure 3.27, right). This reaction served as a reference case where all syb (49-96) got displaced by syb (1-96). Taking this reference into account the anisotropy results were analyzed. The following points were considered.

- At the moment of syb wt (1-96) addition (at end of the 3-minute-incubation) there were free acceptor sites available for syb wt (1-96) binding.

- What happened to the trans complexes that had assembled in the first 3 minutes? Were they disturbed by syb (1-96) binding?

- If yes, were the liposomes detached?
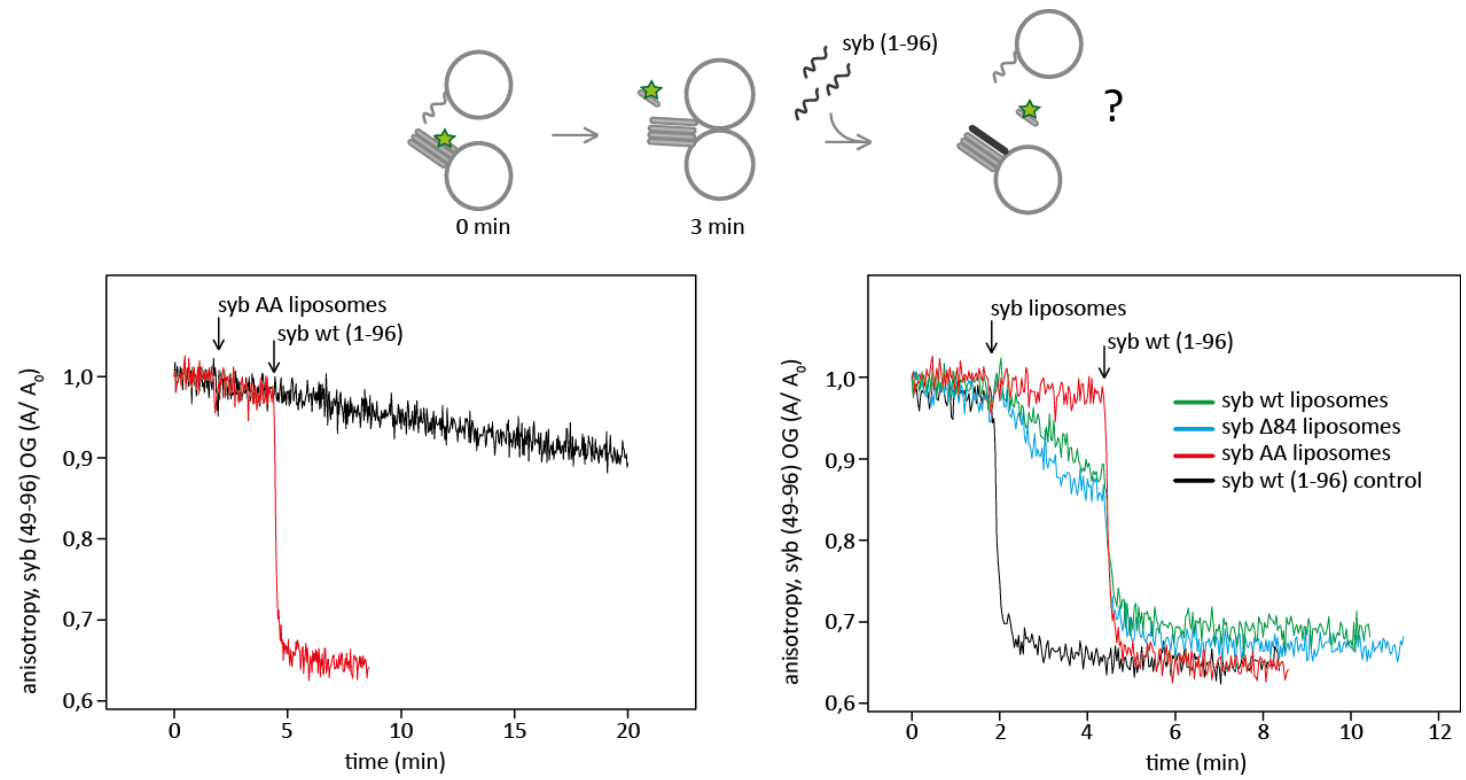

Figure 3.27. Not all of the acceptor complexes are associated with synaptobrevin in the first 3 minutes of mixing. The acceptor liposomes described in figure 3.21 were used. The anisotropy of Oregon Green labeled syb (49-96) was monitored and normalized to its initial value $\left(A_{0}\right)$. Left, acceptor liposomes were mixed with unlabeled syb AA liposomes. After the first 3 minutes, excess amounts of unlabeled syb wt (1-96) was added to the reaction, which is depicted with the red trace. The black trace is the same reaction of syb AA liposomes overlaid from figure 3.21. Right, the same reaction shown on the left is repeated mixing the acceptor liposomes with either of the following: syb AA liposomes (red), syb $\Delta 84$ liposomes (blue), syb wt liposomes (green). After the first 3 minutes of each reaction excess amounts (75-fold) of unlabeled syb wt (1-96) was added. A control reaction in which the acceptor liposomes were directly mixed with syb wt (1-96) is also shown with a black trace. 
Results

In order to answer the above-mentioned questions. An in vitro FCCS assay was developed. Texas Red labeled acceptor liposomes were mixed either with Oregon Green labeled syb $\Delta 84$ liposomes or with Oregon Green labeled syb AA liposomes (see figure 3.28). After the first 3 minutes of mixing, unlabeled excess syb wt (1-96) was added to each reaction. The docking percentages were assessed for each reaction before and after syb wt (1-96) additions. As a control, the same set of experiments were repeated by adding buffer instead of syb wt (1-96).
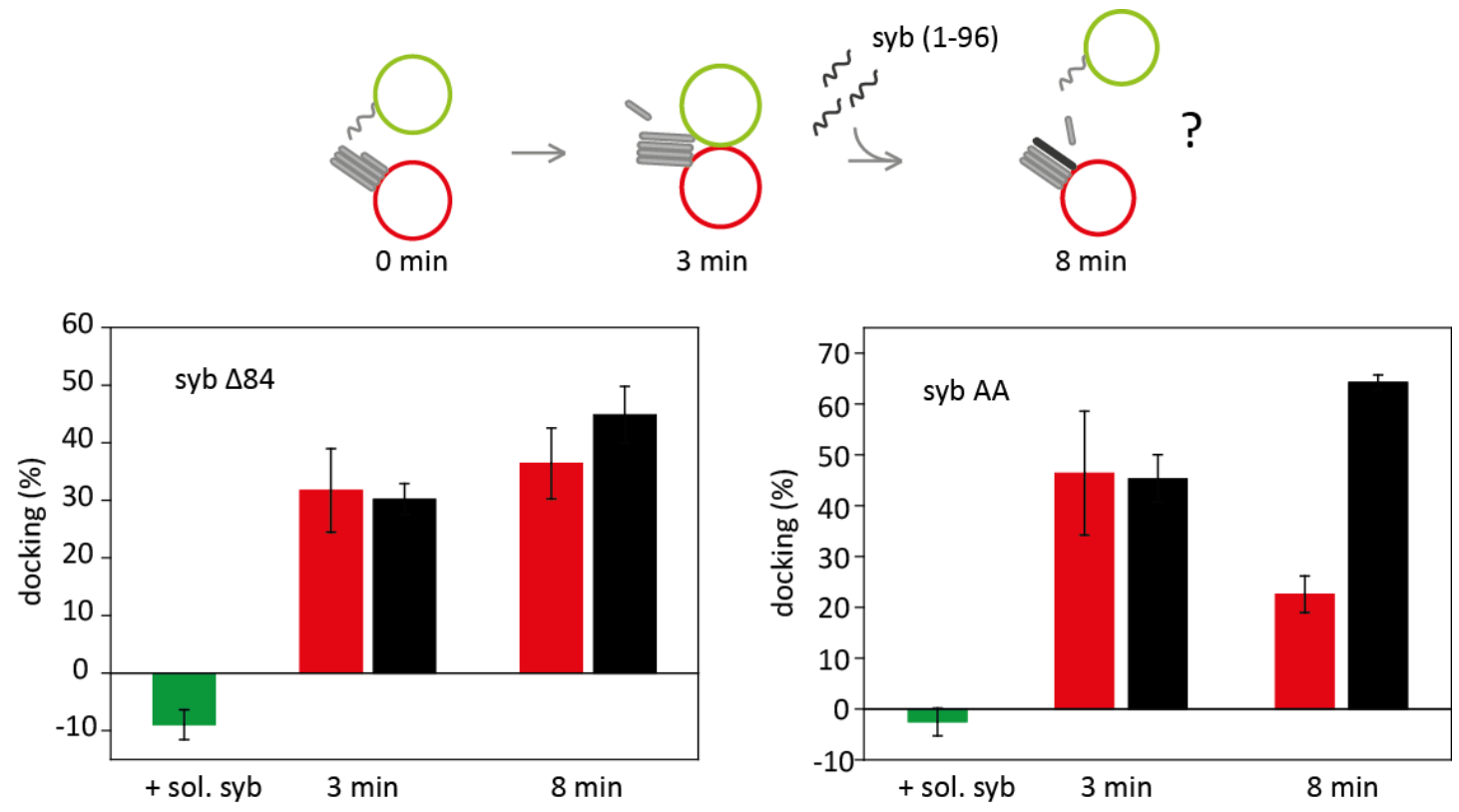

Figure 3.28. Syb wt (1-96) competes with the assembling trans SNARE complexes for the same acceptor sites. Texas Red labeled liposomes were mixed either with Oregon Green labeled syb $\Delta 84$ liposomes (left) or with Oregon Green labeled syb AA liposomes (right). Liposomes were mixed in liposome buffer (total volume $300 \mu \mathrm{L}$ ) shaking at $37^{\circ} \mathrm{C}$. After the first 3 minutes of mixing, $10 \mu \mathrm{L}$ sample was collected and analyzed. At this time point, an excess amount (75-fold) of syb wt (1-96) was added to the reaction and incubated for another 5 minutes. At the end of this incubation, the last sample was collected and analyzed. These experiments are depicted with red bars. In parallel, control reactions with buffer additions, instead of syb wt (1-96), were prepared and depicted with the black bars. Green bars show the control reactions for SNARE-dependent-docking, where excess syb wt (1-96) had been present from the start of the docking reaction. Each bar represents the average fluorescence cross correlation calculated for three independent reactions and error bars represent the standard deviation.

These results showed that the acceptor liposomes stayed docked to syb $\Delta 84$ liposomes even after syb wt (1-96) addition. Syb wt (1-96) prevented further docking 
of syb $\Delta 84$ liposomes but did not detach already docking ones. This indicated that syb wt (1-96) occupied the free acceptor sites that syb $\Delta 84$ would have bound to. Interestingly, in the reactions with syb AA liposomes, syb wt (1-96) lowered the docking percentages of syb AA liposomes. This suggested that in addition to the free acceptor sites, syb wt (1-96) bound to the acceptor complexes that were already associated with the syb AA mutant. Such an interaction might have inhibited the loose zippering of the syb AA trans SNARE complexes and detach the syb AA liposomes partially. This observation raised the necessity for additional experiments to verify whether syb wt (1-96) could displace syb AA monomers that were associating to the acceptor complexes. 
Results 


\section{Discussion}

\subsection{Considerations for constructing an in vitro reconstitution system to study trans SNARE complexes}

This study strove to construct a biochemically well-defined reconstitution system in which the dynamics of the synaptic trans SNARE complexes could be studied. Up to now, partially assembled SNARE complexes were spotted via various in vitro approaches, but their characteristics were not addressed fully [130,133]. In most of the cases, the read-outs of the liposome fusion reactions were regarded as the indications for the existence of trans complexes [75, 160]. Other approaches involved isolating trans complexes by solubilizing them in detergents with the risk of converting them spontaneously to cis complexes [130]. Here, careful considerations were given to develop a system that monitors the SNARE complexes directly as they assembled between two opposing membranes.

In three reconstitution systems developed in this study, purified SNARE monomers were mixed to generate trans complexes with the deletion mutant of synaptobrevin (syb $\Delta 84$ ) between artificial membranes. When designing these different approaches an important point to consider was the nucleation reaction. For the ternary complex nucleation, the synaptic vesicle SNARE, synaptobrevin requires a transient, preassembled acceptor complex of the presynaptic membrane SNAREs, syntaxin and SNAP-25 with 1:1 stoichiometry (see section 1.1 .3 ). So far, it was only possible to stabilize this complex in vitro by co-purifying it with a truncated synaptobrevin fragment, syb (49-96). The first two reconstitution systems of this study avoided using this stabilizing fragment in the trans complex nucleation steps. Without the 


\section{Discussion}

stabilized acceptor complexes, SNARE monomers were let to nucleate with slower nucleation kinetics, though into more native forms of trans complexes. Due to the slow nucleation in these systems, monomers were mixed during long pre-incubations to allow them to assemble into trans complexes (see figure 3.1 and figure 3.4). Previous studies claimed that similar pre-incubations generated trans complexes between docking liposomes $[69,70]$. In fact, the first reconstitution system of this study involved very similar compositions of SNARE monomers with such methods.

However, even if SNAREs were pre-incubated, either starting from purified complexes of syntaxin and SNAP-25 (see figure 3.3) or by adding soluble SNAP-25 (see figure 3.8), synaptobrevin nucleation was highly inefficient. Moreover, it was previously demonstrated that adding excess amounts of soluble SNAP-25 to small liposomes of syntaxin and synaptobrevin or to large dense core vesicles did not circumvent this problem of nucleation $[143,161]$. In any case, the reconstitution systems would be dominated by 2:1 complexes, with the acceptor site being blocked by syntaxin, consistent with the previous studies of SNAREs in solution or on small liposomes [25, 30,49].

The observations obtained in the first two reconstitution systems verified that in the absence of the regulatory factors (like Munc13 and/or Munc18) that stabilize the assembly intermediates, nucleation of SNARE zippering occurs at very slow rates. For this reason to generate SNARE complexes the monomers must have been preincubated, like it was also done in the previous studies $[70,75,162]$. The problem with this pre-incubation approach was that these studies monitored only the fusion

of the pre-incubated SNARE liposomes. However, in these recordings the very same outcome of liposome fusion might have resulted from SNARE zippering reactions following different nucleation paths via different assembly intermediates [163]. This possibility demands particular attention since these studies deduced protein-protein interactions that regulate SNARE zippering only from the liposome fusion read-outs. When comparing such read-outs from different studies, it must be born in mind that the nucleation conditions might have been very different in each case. Essentially, this realization might explain why different studies suggest different roles for the same 
regulatory factors acting on SNARE zippering. The critical phase that needs to be investigated is the conformational cycles that synaptic SNAREs undergo at each step that leads to regulated exocytosis. Rather than 'already-happened' incubations, this cycle needs to be recorded in real-time. A probe to monitor the dynamics of the trans complexes between two opposing membranes would illustrate these conformational cycles more clearly.

\subsection{Introducing a reconstitution system to study two types of trans SNARE complexes}

In the search for an above-mentioned probe, the first two strategies raised the necessity to use stabilized acceptor complexes for synaptobrevin nucleation. For this reason, such complexes were used in the final reconstitution system (see section 1.1.3 and [55]) and the long sought probes for the direct monitoring of the trans complexes were successfully introduced. With the two hydrophobic layer mutants of synaptobrevin, the deletion mutant (syb $\Delta 84$ ) and the substitution mutant (syb AA), trans complexes were successfully generated between docking and not fusing liposomes. Both the complex assembly and disassembly cycle as well as the liposome docking were separately monitored in this system and correlating conclusions were realized. These read-outs suggested that the trans complexes that were generated using syb $\Delta 84$ and syb AA mutants exhibited different configurations. The characteristics of these two different types of trans complexes and their interactions with the disassembly machinery are discussed in the following sections.

\subsubsection{Insights into trans SNARE complex zippering}

The first set of experiments with the syb $\Delta 84$ mutant confirmed that the deletion in the $+8^{\text {th }}$ layer of the synaptobrevin SNARE motif prevented large liposome fusion and caused an accumulation of docked liposomes in a time-dependent manner (see figure 3.10 and figure 3.24). One of the novelties of this reconstitution system was that the assembly of the complexes on the docking liposomes could be monitored in real-time (see figure 3.11). A FRET-based bulk assay was developed to serve this purpose. 


\section{Discussion}

Because in this assay the acceptor complexes were stabilized with the syb (49-96) fragments, no other SNARE complex assembly intermediate would nucleate. Therefore, the FRET signal reported exclusively on the zippering syb $\Delta 84$ trans complexes. However, using the same assay to investigate the SNARE interactions involving the syb AA mutant between non-fusing large liposomes did not provide evidence on SNARE complex formation. Even if the mutation was downstream of the labeling positions, the bulk FRET and fluorescence anisotropy assays did not reveal any N-terminal SNARE associations (see figure 3.22 and figure 3.23). These observations were intriguing because the non-fusing large liposomes were docking in a syb AA dependent manner (see figure 3.26).

Another powerful feature of the system was that it was possible to implement other regulatory factors. Since the trans complexes resided on the membranes, they were the ideal membrane-bound substrates for these factors. For example, $\alpha$-SNAP was shown to have a high affinity for the membrane and support the disassembly of the membrane-bound complexes 20 -fold more efficiently [99]. NSF/ $\alpha$-SNAP and tetanus neurotoxin were incorporated into this system with this motivation and helped address the puzzle on the SNARE interactions involving the two mutants.

The results presented with the gel-based experiments with tetanus toxin showed that almost all of the syb $\Delta 84$ were associated with the acceptor complexes and zippered into toxin-resistant trans complexes (see the $3^{\text {rd }}$ and $4^{\text {th }}$ reactions in figure 3.17 and $[38,109])$. The same assay also validated the trans SNARE interactions between syb AA and the acceptor complexes (see $3^{\text {rd }}$ and $4^{\text {th }}$ reactions in figure 3.23 ), though the fluorescence labeling positions were not suitable to track them. In fact, the partial toxin resistance of the syb AA trans complexes pointed to structures that might be more loosely assembled compared to syb $\Delta 84$ trans complexes. These findings introduced the trans complexes of syb AA as very different, loose structures in comparison to the tightly zippered trans complexes of syb $\Delta 84$ between tightly docked large liposomes [149]. 
The experiments shown in the figures 3.25 and 3.26 highlighted two reactions that caused docking and operated sequentially. The first reaction was the SNARE zippering which was essential for the initiation of liposome docking. And the second one was the irreversible docking over time which was not reverted by the disassembly of the trans complexes. In the control experiments where SNARE zippering was inhibited, no docking was observed no matter how long the synaptobrevin and acceptor liposomes were mixed (see the green bars in figure 3.25 and the orange bars in figure 3.26). But in the experiments without this inhibition, once the SNARE nucleation had started, the liposome docking followed and after a certain time point it became independent of the SNARE zippering reaction. Understanding when this time point arrived was crucial in deducing the extent of zippering from the readings on the extent of liposome docking.

In the previous liposome fusion experiments on large liposomes, an initial lag phase was discovered [38]. During the first three minutes of liposome mixing no fusion occurred, even though SNARE zippering had started immediately at the time of mixing. It was concluded that during this lag phase, SNARE nucleation occurs and it takes about three minutes to start liposome fusion. The FCCS experiments of this present study took advantage of these findings. As shown with the anisotropy experiments SNARE zippering of the syb $\Delta 84$ proceeded very similar to that of the wild type synaptobrevin (see figure 3.21 ) until the $+8^{\text {th }}$ layer of the SNARE motif. However, after this lag phase syb $\Delta 84$ might have led to the irreversible docking of the liposomes instead of their fusion as the wild type synaptobrevin did. The FCCS experiments addressed this possibility by incorporating NSF in the liposome mixing reactions before this lag phase ended. Furthermore, the trend of irreversible docking was also tested with the syb AA liposomes.

These experiments showed that NSF was not "quick enough" preventing the irreversible syb $\Delta 84$ liposome docking even when present since the beginning of the liposome mixing. This indicated that the fast SNARE zippering until the $8^{\text {th }}$ layer accommodated irreversible liposome docking even in the presence of NSF. Surprisingly, syb AA liposomes were prevented from docking by NSF (compare the 0- 


\section{Discussion}

minute-incubation reactions in figures 3.25 and figure 3.26). Unlike the previous observation, this outcome suggested that zippering was arrested before the irreversible docking started and in this time window NSF was able to detach the syb AA liposomes. Both for syb AA and for syb $\Delta 84$ experiments, the later NSF was added to the liposome mixing incubations the less potent it became in preventing the irreversible docking. However, only in syb AA experiments, NSF was able to detach once docked liposomes. The best explanation for these findings was that the freshly nucleating complexes of syb AA were zippering more slowly into perhaps more loose structures and hence were susceptible to NSF-mediated disassembly before they could bring the large liposomes together.

These sets of FCCS experiments were very compelling in elucidating the different configurations of tight syb $\Delta 84$ and loose syb AA trans complexes. They demonstrated that in this reconstitution system it is possible to stabilize physiologically relevant intermediates. These intermediates might resemble previously reported tight and loose trans complexes $[47,108]$. Two types of trans complexes arrested at different regions of the SNARE motif might allow to investigate the regulatory factors exerting their effects at different stages of the zippering reaction. For instance, possible effects of Munc18 and complexin on tightly zippered trans complexes [70] and on loosely zippered complexes [164] might be addressed, respectively (see section 4.3).

Nevertheless, the questions remained: how far does the zippering progress in the trans complexes before the liposomes are irreversibly docked? Especially in the syb AA trans complexes, what causes the arrest in zippering? Recent experiments using optical tweezers set-ups reported that the $\mathrm{N}$ - and $\mathrm{C}$-terminal zippering have different energies due to an energy minimum around the 0-layer. It was also suggested that the increasing repulsion between the membranes might contribute to the slow zippering around this region [117]. This present study with the syb $\Delta 84$ and syb AA trans complexes has shown that SNARE-intrinsic factors, rather than the membrane repulsing each other contributed more to the arrest in zippering. This was demonstrated with the irreversible docking of liposomes even if the SNARE zippering was arrested at the very N-terminal region of the SNARE motif (see figure 3.26). 
However, it was not as trivial to address where the energy minimum or a molecular switch region was in the SNARE motif that might divide the zippering in different energy levels. In addition to the previous study which involved optical tweezers, comprehensive point mutation studies on SNAP-25 and synaptobrevin SNARE motifs also put forward the concept of a trigger site in zippering (see section 1.2.1). So far, the critical areas that have been studied include the region between $+4^{\text {th }}$ and $+6^{\text {th }}$ layers $[114,115]$, the 0 -layer $[117,165]$ and the $-3^{\text {rd }}$ layer $[116]$.

A common pitfall of the optical tweezers studies was that the conclusions on SNARE zippering mechanisms were only based on the unzipping reactions [117, 119, 166]. This was essentially in contradiction with the hysteresis that the dissociated SNARE monomers exhibited [54]. It was clearly established earlier that the SNARE monomers follow different assembly and disassembly pathways. Even though Min et al. addressed this issue and looked at both unzipping and re-zipping of the SNARE complexes [119], additional methods are needed to study the potential trigger/switch sites of SNARE motifs.

This present study pointed to a potential switch region around the $-3^{\text {rd }}$ layer by having taken a closer look into the previously introduced double-alanine substitutions in synaptobrevin SNARE motif [116]. Further questions were asked to study the nature of the loose trans interactions that the syb AA mutant was involved in. Were they too loose to be dissociated when excess soluble syb (1-96) fragments competed for the same acceptor complexes? If this competition was won by soluble syb (1-96), did it detach the liposomes? The experiments displayed in figure 3.27 suggested that soluble syb (1-96) might in fact interfere with syb AA zippering and dissociate the loose complexes. Such a loose complex configuration might involve a half-zippered, C-terminally unfolded syb AA SNARE motif. This configuration is plausible considering the fact that the double alanine substitutions are found in a region which is predicted to be an $\alpha$-helical trigger site [116]. When this region is mutated to alanines, the $\alpha$-helicity and hence the triggering function for further zippering might have been lost. Such a structural destabilization in the otherwise helical region might offer a possible explanation why a downstream mutation led to an upstream arrest in 
Discussion

SNARE zippering, and hence made it impossible to track with fluorescence spectroscopy (see figure 3.20 and figure 3.22).

\subsubsection{Insights into NSF-mediated trans SNARE complex disassembly}

This study provided the first set of direct evidence on the disassembly of the trans SNARE complexes that reside between two opposing membranes. All of the tightly zippered syb $\Delta 84$ trans complexes between docked large liposomes were disassembled (see the right panel in figure 3.12, and the 2 nd reaction in figure 3.17). Similarly, the trans interactions of syb AA which displayed tetanus toxin resistance, were also shown to be disassembled in the presence of NSF and $\alpha$-SNAP (see $2^{\text {nd }}$ reaction in figure 3.23). It was clearly demonstrated that NSF was able to disassemble every complex that had assembled in this reconstitution system, including the acceptor complexes (see figure 3.16).

These results contradicted with the previous proposals suggesting that trans complexes might be resistant to the disassembly (see section 1.2.4 and [133]). In fact, this contradiction presents a good example of how indirect measurements of liposome fusion may result in misleading interpretations on SNARE interactions. In the study which put forward the NSF-resistance proposal, it was assumed that trans complexes were forming between the liposomes during the overnight incubation at low temperature. The existence of these trans complexes was not well documented, hence the study failed to address the disassembly of these complexes directly. The proposal speculated that the trans complexes might have been resistant to NSF because

- $\alpha$-SNAP might not recognize them.

- NSF might be sterically excluded from the space between two docking liposomes.

- Even if the complexes were disassembled, the monomers might immediately re-assemble. 
In the present reconstitution system, by directly monitoring the assembly/ disassembly cycles, it was possible to reject almost all of these points. First, it was shown that $\alpha$-SNAP is capable of binding to the trans complexes (see Appendix A8). Second, since the acceptor complexes were also disassembled the SNARE monomers were demonstrated not to be re-assembling due to the hysteresis of the SNARE monomers (see figure 3.15, and [49]). And lastly, the complexes were not sterically excluded from NSF/SNAP binding. Even the bulky full-length complexes with the Nterminal domain (Habc domain) of syntaxin were disassembled either in trans or in cis configuration (see figure 3.14 and [152]).

The results reporting on the trans complex disassembly in this study agrees well with the previously proposed a "protective reassembly" model $[20,108]$. According to this model NSF disassembles the SNARE complexes in various configurations of SNARE zippering intermediates, as long as its co-factor SNAP recognizes them. Even the nonfusogenic complexes with the Qabab compositions have been shown to be disassembled by NSF [40]. The model presumes a continuous assembly and disassembly cycle of the trans complexes at the pre-synaptic site until the fusogenic complexes are stabilized or are protected from NSF by the regulatory factors like Munc18. Possibly with the help of this "proof-reading" mechanism, membrane fusion occurs only when the correct complexes assemble. Although indirect, there are recent data supporting this view $[75,129]$.

The findings on the full-length trans complex disassembly raised interest. According to the most recent 3D reconstruction of the $20 \mathrm{~S}$ particles, three $\alpha$-SNAP molecules bind around the SNARE complex bundle and one NSF molecule associates with the membrane distant $\mathrm{N}$-terminal region of the SNAP-SNARE complexes [103]. However, at the point of this association, the N domains of NSF were not fitted properly to the $\mathrm{N}$-terminal end of the SNARE bundle in the electron microscopy map. Thus, the region where NSF and the syntaxin Habc domain sit remained a mystery. 
Discussion

\subsubsection{Irreversible docking of large liposomes}

Another inexplicable phenomenon discovered in this study of trans complexes was concerning the dynamics of the liposome membranes. Liposome docking was initiated by the assembly of trans complexes, however it was not reversed upon the disassembly of the complexes (see figure 3.24). Instead, an irreversible docking state developed in time and liposomes became arrested tightly pressed to each other as described previously [149]. It seemed that no matter how far the SNARE zippering progressed and got arrested, the liposomes were caught in this unique docking state (see figure 3.25and figure 3.26). Moreover, it was postulated that in this state the bilayer-bilayer contact site might have been dehydrated [149]. Here, it is tempting to speculate that such a dehydrated state might indeed correspond to a contact site which is held together by hydrophobic adhesion. Israelachvili and co-workers have discussed a model for this type of interaction of bilayer-bilayer contact sites [167169]. According to this model, before they are brought in contact, two unstressed bilayers are under strong repulsion due to electrostatic/hydration forces. And these forces mask the weak van der Waals attraction. Once the bilayers are pressed together in the surface force apparatus, they become stressed or stretched and hence exhibit local bilayer deformations. Particularly, thinning of the opposing outer layers exposes the hydrophobic chains of the lipids that strongly attract each other. Furthermore, this model describes such deformations similar to first-order phase transitions [167]. An independent line of research on giant liposomes provided evidence that might support this model. It was shown that the adhesion of giant liposomes was coupled to the membrane phase separation of the lipids with negative spontaneous curvature (e.g. PE) [170]. If Israelachvili's model holds true also in the giant liposome system, membrane domains rich in lipids like PE might expose more hydrophobic regions causing the adhesion of the opposing bilayers.

Taken together with the speculative hydrophobic adhesion model, the irreversibly docking large liposomes of this study might present a similar adhesion state. By lowering the content of the lipids with negative spontaneous curvature of the large liposomes, the existence of such a state might be further tested (Dr. H. Jelger 
Risselada, personal communication). It might be worthwhile to check if these liposomes would detach from each other after the trans complex disassembly step.

Multiple docking of the liposomes was considered as an alternative explanation for the irreversible docking phenomenon (see figure 3.24). If multiple liposomes were docking to each other, trans complexes might have been trapped in the inner contact sites. Such complexes would have been protected from NSF-mediated disassembly, and perhaps would have kept the multiple liposomes docked together. Nonetheless, it has been shown that this was not the case by different lines of evidence. Firstly, the FCCS data was re-examined and no indications of multiple docking was found (Iman Kattan and Dr. Peter Jomo Walla, personal communication, also see [154]). Secondly, experiments with tetanus neurotoxin clearly showed that there were almost no NSFresistant trans complexes on the docked liposomes (see the $2^{\text {nd }}$ reaction in figure 3.17).

Although it was possible to speculate on the interactions keeping this docked state of liposomes together, the physiological role of such a state remains to be a question mark. If this structure constitutes an intermediate of the fusion pathway in the cell, how does the cell cope with this low-energy intermediate?

\subsection{Further studies on trans SNARE complexes}

The biochemical reconstitution system that was introduced in this study provided powerful means to study the dynamics of the trans SNARE complexes. In this system it was possible to monitor directly both the SNARE complex assembly/disassembly cycles and how this cycle governs liposome membrane dynamics. This established system now offers the valuable tools to answer further questions on trans SNARE complex regulation that comprise the critical steps in synaptic vesicle fusion.

One point can be addressed rather swiftly. Without changing the components of the reconstitution system and using fluorescence anisotropy, the extent of zippering in syb AA trans complexes would be determined. An assay that monitors the binding of 


\section{Discussion}

several C-terminally truncated soluble syb AA fragments to the acceptor complex might achieve this goal.

Another set of future experiments might require further optimizations of the reconstitution system. Implementing the early (e.g. Munc13 and Munc18) and late regulators (e.g. synaptotagmin and complexin) into this system, a comprehensive investigation could unravel the sequence of events leading to neuronal exocytosis (see figure 4.1). In this way, the current models of these steps would be challenged (see figure 1.5). Since this system successfully arrests otherwise very transient trans complexes, it would be quiet possible to include or subtract any of these regulatory components and study their abilities in rescuing these complexes. So far, two such trapped trans SNARE complexes with very different configurations made available using the hydrophobic layer mutants of synaptobrevin, syb $\Delta 84$ and syb AA. A strong candidate for further methods to generate similar trans complexes might be a hydrophobic layer mutant of SNAP-25 which has an alanine substitution mutant around its $+5^{\text {th }}$ layer [114].

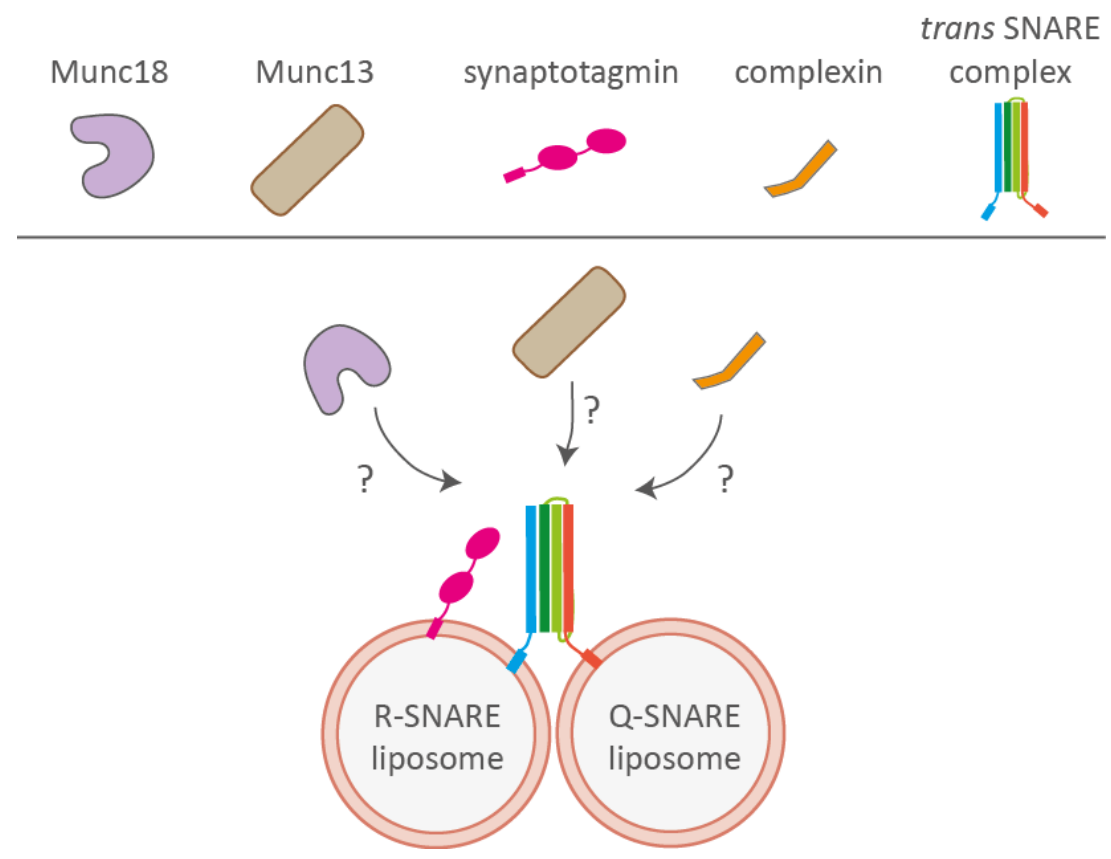

Figure 4.1. Schematic representation of the possible modifications to the reconstitution system of the trans SNARE complexes. 
Discussion 


\section{Appendix}

\section{A1. Synaptobrevin cDNAs compared}

The comparison of the cDNA sequences of synaptobrevin (syb wt) and the deletion mutant (syb $\Delta 84$ ) are compared as an example. The same analysis was performed as new SNARE variants were generated (see Table 2.2).

syb wt (1-116) cDNA, Rattus norvegicus

atg tcg gct acc gct gcc acc gtc ccg cct gcc gcc ccg gcc ggc gag ggt ggc ccc cct gca cct cct cca aat ctt acc agt aac agg aga ctg cag cag acc cag gcc cag gtg gat gag gtg gtg gac atc atg agg gtg aat gtg gac aag gtc ctg gag cgg gac cag aag cta tcg gaa ctg gat gat cgc gca gat gcc ctc cag gca ggg gcc tcc cag ttt gaa aca agt gca gcc aag ctc aag cgc aaa tac tgg tgg aaa aac ctc aag atg atg atc atc ttg gga gtg att tge gec atc atc ctc atc atc atc atc gtt tac ttc agc act taa

syb (1-116) S28C $\Delta 84$ cDNA, optimized for expression in E.coli atg tct gct acc gct gct acc gtt ccg ccg gct gct ccg gct ggt gaa ggt ggt ccg ccg gct ccg ccg ccg aac ctg acc tgc aac cgt cgt ctg cag cag acc cag gct cag gtt gac gaa gtt gtt gac atc atg cgt gtt aac gtt gac aaa gtt ctg gaa cgt gac cag aaa ctg tct gaa ctg gac gac cgt gct gac gct ctg cag gct ggt get tct cag ttc gaa acc tct gct gct aaa aaa cgt aaa tac tgg tgg aaa aac ctg aaa atg atg atc atc ctg ggt gtt atc tgc get atc atc ctg atc atc atc atc gtt tac ttc tct acc taa

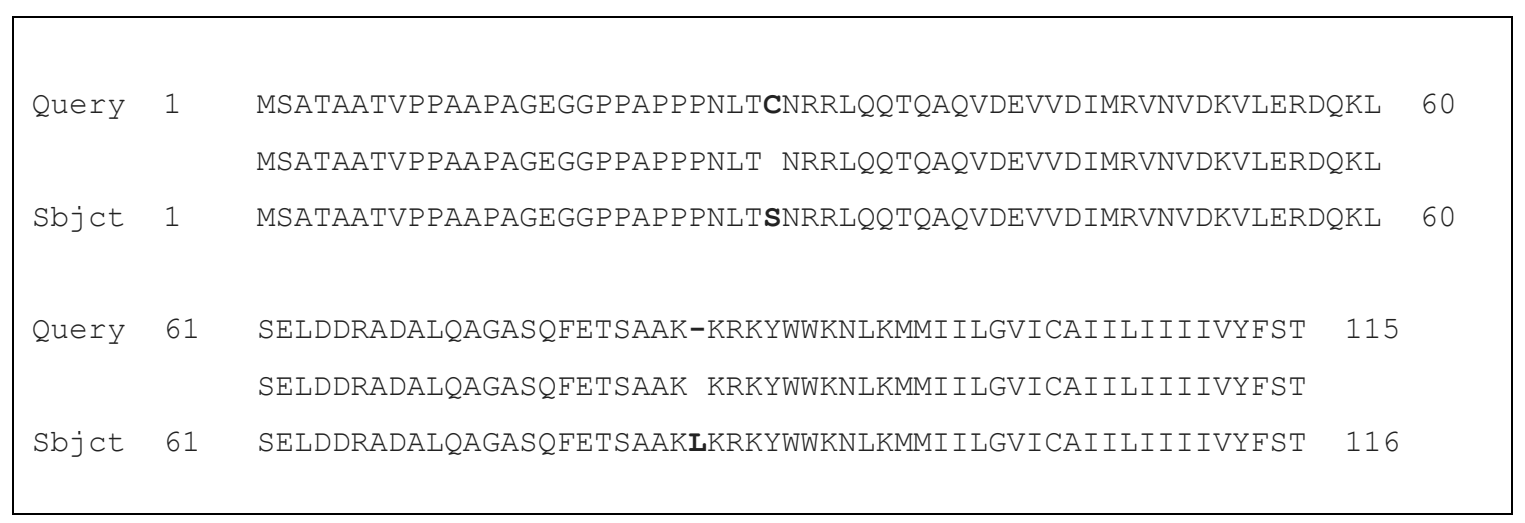

Above sequences were translated using the translate tool (ExPASy, SIB) and aligned using the blastp suite (NCBI). 'Sbjct 1' depicts syb wt sequence, whereas 'Query 1' depicts syb $\Delta 84$ sequence. 


\section{A2. Oligonucleotides used to generate neuronal SNARE variants}

\subsubsection{Primers for sub-cloning syxFL from $p E T 28 a$ to $p T X B 1$ vector}

Forward Primer, contains an NdeI cut site:

5' ggtggtcatatgatgaaggaccgaacccagg 3'

Reverse Primer, contains a SapI cut site:

5' ggtggttgctcttccgcatccaaagatgccccgatg 3'

\subsubsection{Primers for generating syb (1-116) S28C, I45A, M46A contruct}

Forward Primer 'A', contains NdeI cut site:

5' gtattacatatgatgtcggctaccgctgc 3'

Reverse Primer 'B':

5' caccacctcatccactgggc 3'

Forward Primer ' $\mathrm{C}$ ':

5' agactgcagcagacccagg 3'

Reverse Primer 'D', contains Xhol cut site:

5' gacagcctcgagttaagtgctgaagtaaacgatg 3'

\section{A3. Expression and purification of syx (183-288)}

Syntaxin (183-288) was one of the exceptional constructs that required further optimizations with its purification protocol. Bacterial expression, lysis and protein extraction from the cell lysate were performed as explained in 2.2.2.2 and 2.2.2.3. Additionally, wash and elution buffers were supplemented with $6 \mathrm{M}$ urea and $3 \%$ $(\mathrm{w} / \mathrm{v})$ sodium cholate. The eluate containing the His-tagged protein was dialyzed overnight at $4^{\circ} \mathrm{C}$ to lower the urea concentration to $2 \mathrm{M}$ in the same buffer composition. Next day, a second dialysis was performed, during which urea was 
Appendix

removed completely and detergent and salt contents were reduced to $2 \%(\mathrm{w} / \mathrm{v})$ and $200 \mathrm{mM}$ respectively. Also, by adding thrombin into the dialysis tubing the His-tag was cleaved. An anion exchange column (Mono Q 10/100 GL, GE Healthcare) was equilibrated with a HEPES buffer (pH 7.4) with $100 \mathrm{mM} \mathrm{NaCl}$ and 1\% CHAPS (Anatrace). After the dialysis, sample was injected to the anion exchange column and was eluted over a linear two step ionic strength gradient at about $35 \mathrm{mS} / \mathrm{cm}$. An example purification profile is shown in figure A.1.

A4. Compositions of the labeled lipid mixes for large liposome preparations with $500 \mu \mathrm{L}$ end volumes

\begin{tabular}{|c|c|c|c|c|c|}
\hline lipid & lipid (\%) & MW & stock $(\mathrm{mg} / \mathrm{mL})$ & $\mathrm{n}(\mu \mathrm{mole})$ & volume $(\mu \mathrm{L})$ \\
\hline PC & $\mathbf{5 0}$ & 770,94 & $\mathbf{2 5 , 0 0}$ & 2,00 & 61,68 \\
\hline PE & $\mathbf{1 9}$ & 746,06 & $\mathbf{1 0 , 0 0}$ & 0,74 & 56,70 \\
\hline PS & $\mathbf{2 0}$ & 812,05 & $\mathbf{1 0 , 0 0}$ & 0,74 & 64,96 \\
\hline chol & $\mathbf{1 0}$ & 386,66 & $\mathbf{1 0 , 0 0}$ & 0,40 & 15,47 \\
\hline TR-DHPE & $\mathbf{1}$ & 1381,85 & $\mathbf{1 , 0 0}$ & 0,04 & 55,27 \\
\hline
\end{tabular}

\begin{tabular}{|c|c|c|c|c|c|}
\hline lipid & lipid (\%) & MW & stock $(\mathrm{mg} / \mathrm{mL})$ & $\mathrm{n}(\mu \mathrm{mole})$ & volume $(\mu \mathrm{L})$ \\
\hline PC & $\mathbf{5 0}$ & 770,94 & $\mathbf{2 5 , 0 0}$ & 2,00 & 61,68 \\
\hline PE & $\mathbf{1 8 , 5}$ & 746,06 & $\mathbf{1 0 , 0 0}$ & 0,74 & 55,21 \\
\hline PS & $\mathbf{2 0}$ & 812,05 & $\mathbf{1 0 , 0 0}$ & 0,74 & 64,96 \\
\hline chol & $\mathbf{1 0}$ & 386,66 & $\mathbf{1 0 , 0 0}$ & 0,40 & 15,47 \\
\hline OG-DHPE & $\mathbf{1 , 5}$ & 1086,25 & $\mathbf{1 , 0 0}$ & 0,06 & 65,17 \\
\hline
\end{tabular}

\begin{tabular}{|c|c|c|c|c|c|}
\hline lipid & lipid (\%) & MW & stock $(\mathrm{mg} / \mathrm{mL})$ & $\mathrm{n}(\mu \mathrm{mole})$ & volume $(\mu \mathrm{L})$ \\
\hline PC & $\mathbf{5 0}$ & 770,94 & $\mathbf{2 5 , 0 0}$ & 2,00 & 61,68 \\
\hline PE & $\mathbf{1 8 , 5}$ & 746,06 & $\mathbf{1 0 , 0 0}$ & 0,74 & 55,21 \\
\hline PS & $\mathbf{1 8 , 5}$ & 812,05 & $\mathbf{1 0 , 0 0}$ & 0,74 & 60,09 \\
\hline chol & $\mathbf{1 0}$ & 386,66 & $\mathbf{1 0 , 0 0}$ & 0,40 & 15,47 \\
\hline NBD-PS & $\mathbf{1 , 5}$ & 985,21 & $\mathbf{1 , 0 0}$ & 0,06 & 59,11 \\
\hline Rho-PE & $\mathbf{1 , 5}$ & 1319,75 & $\mathbf{1 , 0 0}$ & 0,06 & 79,19 \\
\hline
\end{tabular}


Appendix

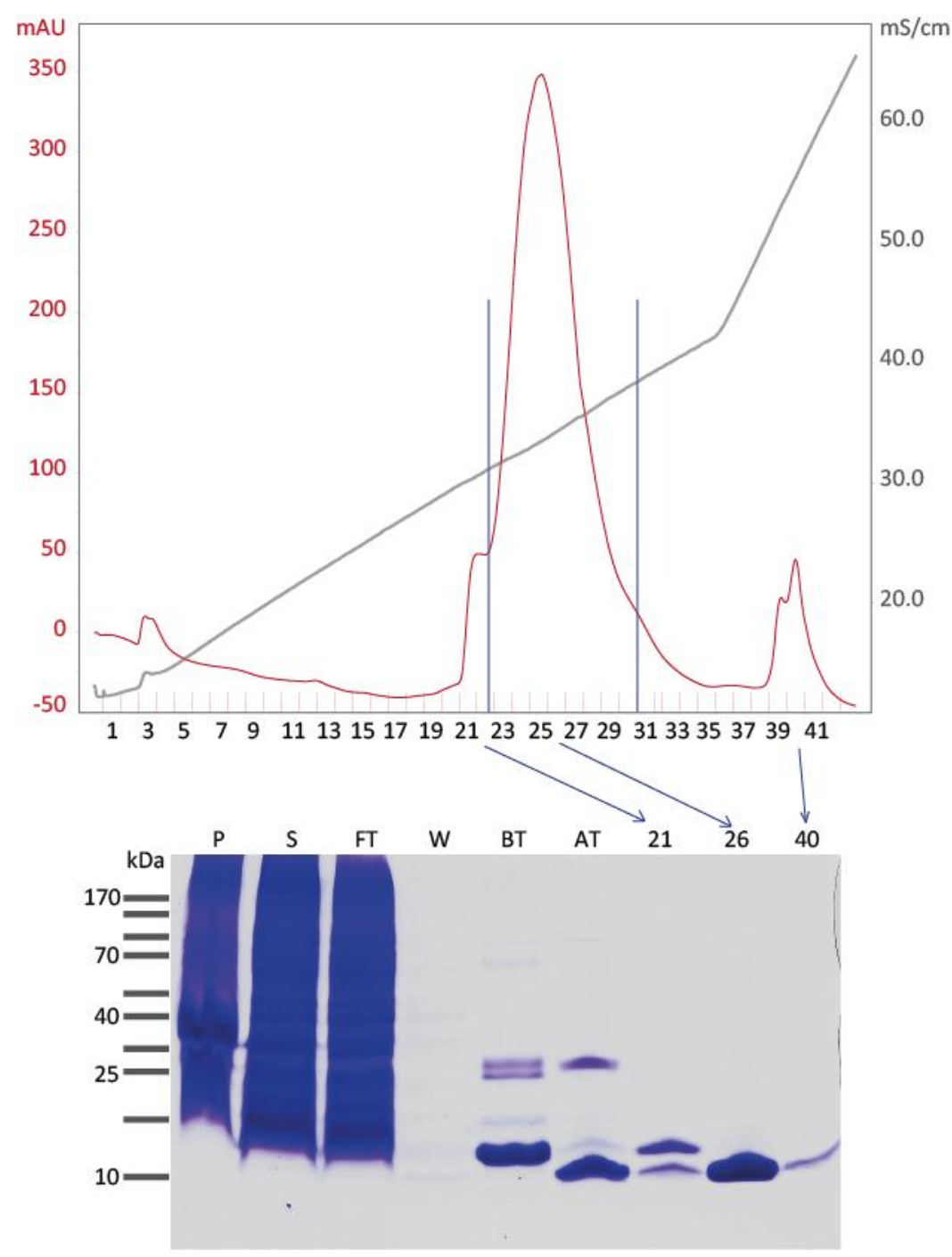

Figure A.1. Expression and purification profile of syx (183-288). Top, ion exchange chromatography was performed using a Mono $Q 10 / 10$ column and the elution profile is shown. Y-axis in red depicts the absorbance (mAU), while $y$-axis in gray shows conductivity (mS/cm). $2 \mathrm{~mL}$ elution fractions are numbered on the $x$-axis. Syx (183-288) was eluted around $35 \mathrm{mS} / \mathrm{cm}$ when a $\mathrm{NaCl}$ gradient was applied. Bottom, the peak fractions of the elution profile was evaluated with Tricin-SDS-PAGE. The single band in fraction 26 corresponds to the most purified fraction of syx (183-288). 


\section{A5. NSF/ $\alpha$-SNAP-mediated disassembly of trans SNARE complexes}
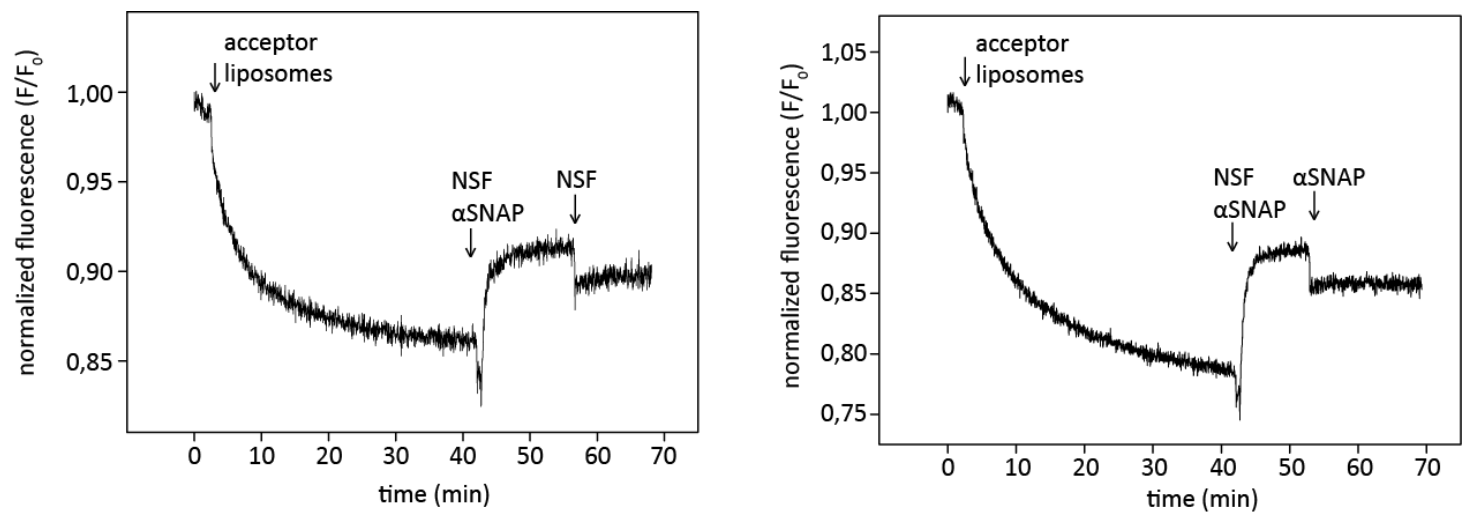

Figure A.2. Trans complexes were disassembled by saturating concentrations of NSF and $\boldsymbol{\alpha}$-SNAP. The experiment shown on the right panel of figure 3.12 was repeated and NSF (right) or $\alpha$-SNAP (left) concentrations were doubled by subsequent additions after the disassembly reactions.

\section{A6. Reconstitution of synaptobrevin mutants in large liposomes}

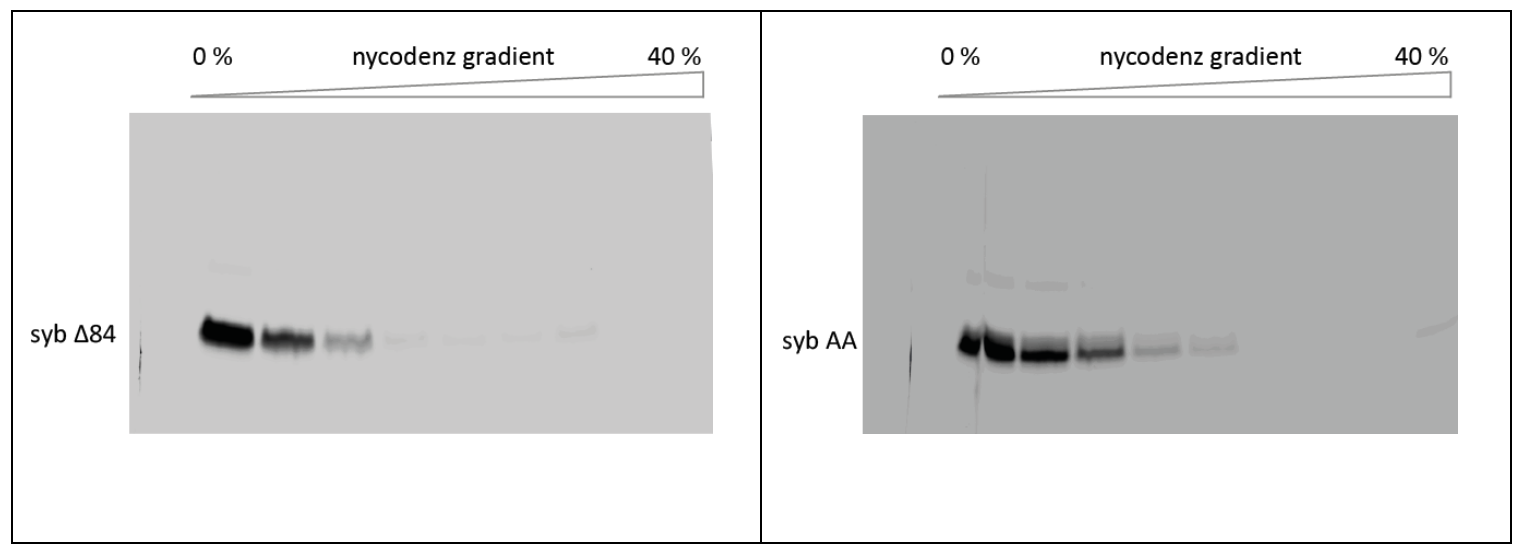

Figure A.3. Both deletion (syb $\Delta 84$ ) and substitution (syb AA) mutants labeled with Oregon Green (at their $\mathbf{2 8}^{\text {th }}$ residues) were reconstituted in large liposomes successfully. The experiment shown on the right panel of figure 3.7 was repeated with fluorescently labeled syb $\Delta 84$ (right) and syb AA (left). The Nycodenz gradient fractions were analyzed by Tricin-SDS-PAGE and the gel was scanned for fluorescence (Fujifilm scanner, FLA-7000). 


\section{A7. Effect of $\alpha$ SNAP on partial SNARE complex zippering}
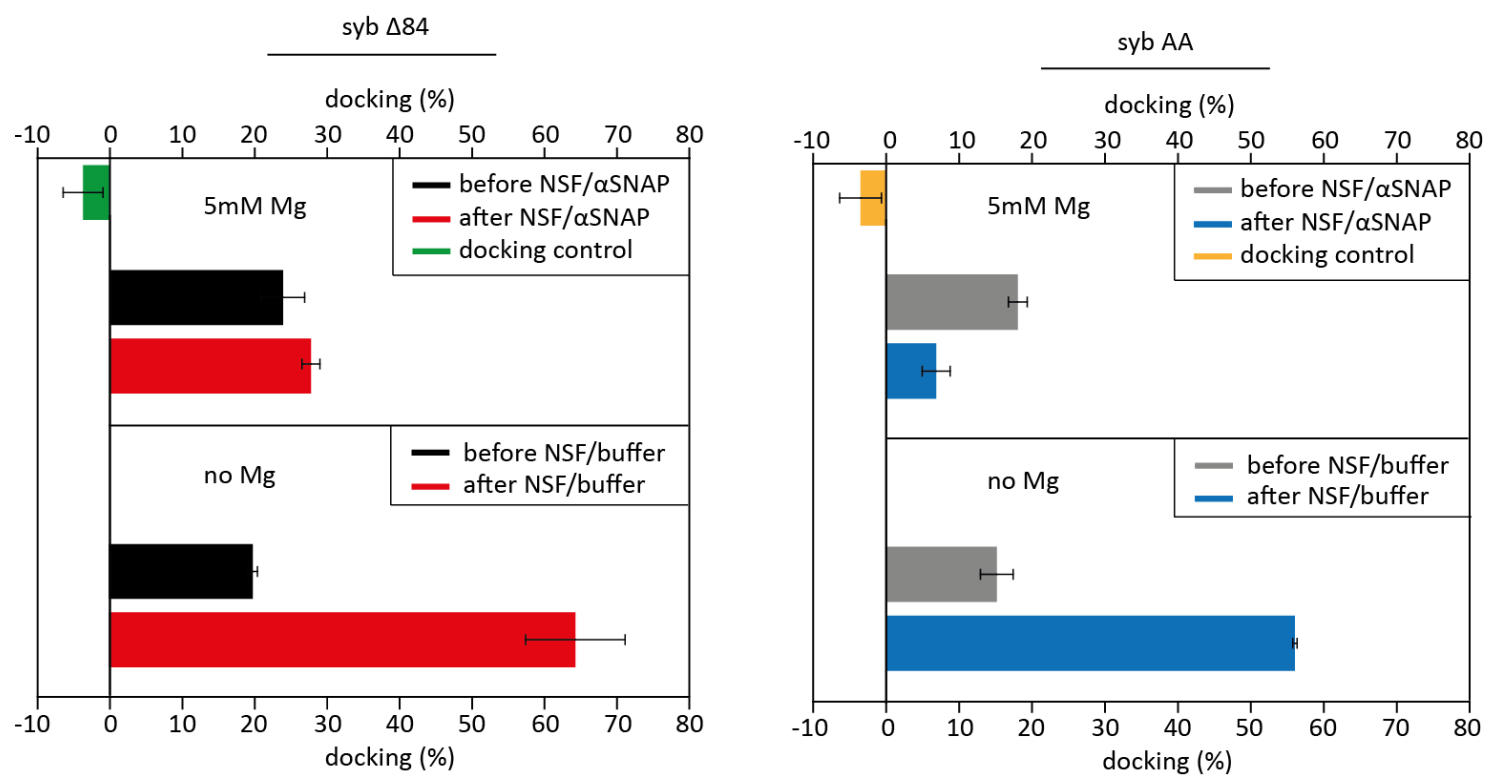

Figure A.4. $\alpha$-SNAP has a negative effect on SNARE-mediated large liposome docking. The 0-minute-incubation experiments which are described in figure 3.25 and figure 3.26 are repeated by using buffer instead of $\alpha$-SNAP. Data analysis and representation were done exactly the same as explained above.

\section{A.8. Trans complex disassembly monitored via fluorescence anisotropy}

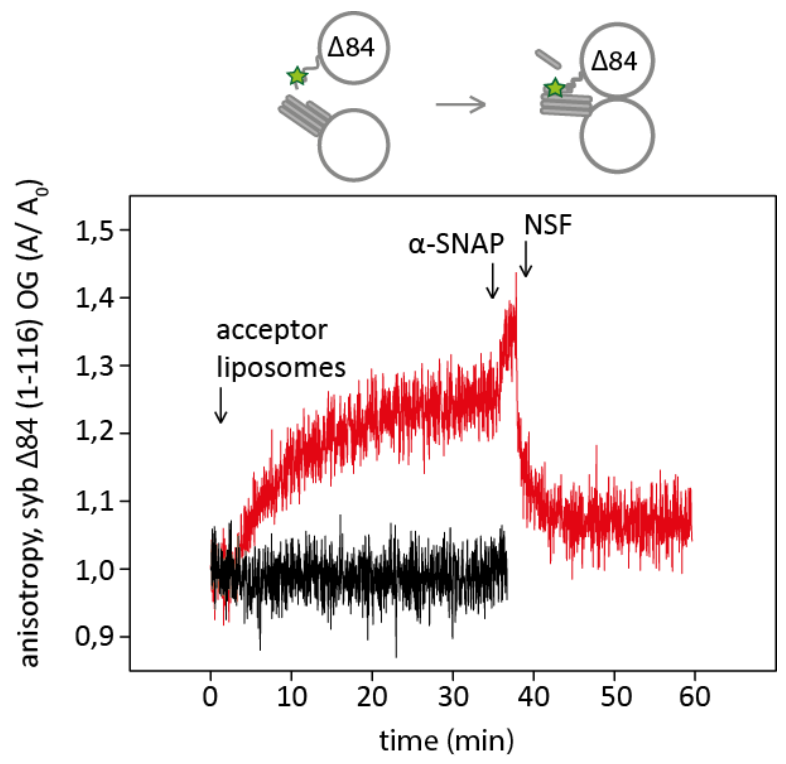

Figure A.5. NSF-mediated disassembly of trans SNARE complexes monitored via fluorescence anisotropy. The experiments which are depicted in the right panel of figure 3.22 were repeated by adding NSF $(70 \mathrm{nM})$ and $\alpha$-SNAP $(1 \mu \mathrm{M})$. 


\section{Bibliography}

1. Palade, G., Intracellular aspects of the process of protein synthesis. Science, 1975. 189(4200): p. 347-58.

2. Bonifacino, J.S. and B.S. Glick, The mechanisms of vesicle budding and fusion. Cell, 2004. 116(2): p. 153-66.

3. Novick, P., C. Field, and R. Schekman, Identification of 23 complementation groups required for post-translational events in the yeast secretory pathway. Cell, 1980. 21(1): p. 205-15.

4. Yamasaki, S., et al., Botulinum neurotoxin type $G$ proteolyses the Ala81-Ala82 bond of rat synaptobrevin 2. Biochem Biophys Res Commun, 1994. 200(2): p. 829-35.

5. Wilson, D.W., et al., A fusion protein required for vesicle-mediated transport in both mammalian cells and yeast. Nature, 1989. 339(6223): p. 355-9.

6. Balch, W.E., et al., Reconstitution of the transport of protein between successive compartments of the Golgi measured by the coupled incorporation of $\mathrm{N}$ acetylglucosamine. Cell, 1984. 39(2 Pt 1): p. 405-16.

7. Clary, D.O., I.C. Griff, and J.E. Rothman, SNAPs, a family of NSF attachment proteins involved in intracellular membrane fusion in animals and yeast. Cell, 1990. 61(4): p. 709-21.

8. Wilson, D.W., et al., A multisubunit particle implicated in membrane fusion. J Cell Biol, 1992. 117(3): p. 531-8.

9. Griff, I.C., et al., The yeast SEC17 gene product is functionally equivalent to mammalian alpha-SNAP protein. J Biol Chem, 1992. 267(17): p. 12106-15.

10. Sollner, T., et al., Snap Receptors Implicated in Vesicle Targeting and Fusion. Nature, 1993. 362(6418): p. 318-324.

11. Tagaya, M., et al., Domain structure of an $\mathrm{N}$-ethylmaleimide-sensitive fusion protein involved in vesicular transport. J Biol Chem, 1993. 268(4): p. 2662-6.

12. Sollner, T., et al., A protein assembly-disassembly pathway in vitro that may correspond to sequential steps of synaptic vesicle docking, activation, and fusion. Cell, 1993. 75(3): p. 409-18.

13. Link, E., et al., Tetanus toxin action: inhibition of neurotransmitter release linked to synaptobrevin proteolysis. Biochem Biophys Res Commun, 1992. 189(2): p. 1017-23. 
Bibliography

14. Schiavo, G., et al., Tetanus and botulinum-B neurotoxins block neurotransmitter release by proteolytic cleavage of synaptobrevin. Nature, 1992. 359(6398): p. 8325.

15. Blasi, J., et al., Botulinum neurotoxin A selectively cleaves the synaptic protein SNAP-25. Nature, 1993. 365(6442): p. 160-3.

16. Blasi, J., et al., Botulinum neurotoxin C1 blocks neurotransmitter release by means of cleaving HPC-1/syntaxin. EMBO J, 1993. 12(12): p. 4821-8.

17. Rothman, J.E. and G. Warren, Implications of the SNARE hypothesis for intracellular membrane topology and dynamics. Curr Biol, 1994. 4(3): p. 220-33.

18. Mayer, A., W. Wickner, and A. Haas, Sec18p (NSF)-driven release of Sec17p (alphaSNAP) can precede docking and fusion of yeast vacuoles. Cell, 1996. 85(1): p. 8394.

19. Lin, R.C. and R.H. Scheller, Structural organization of the synaptic exocytosis core complex. Neuron, 1997. 19(5): p. 1087-1094.

20. Hanson, P.I., et al., Structure and conformational changes in NSF and its membrane receptor complexes visualized by quick-freeze/deep-etch electron microscopy. Cell, 1997. 90(3): p. 523-535.

21. Sutton, R.B., et al., Crystal structure of a SNARE complex involved in synaptic exocytosis at 2.4 angstrom resolution. Nature, 1998. 395(6700): p. 347-353.

22. Jahn, R. and T.C. Sudhof, Membrane fusion and exocytosis. Annu Rev Biochem, 1999. 68: p. 863-911.

23. Jahn, R. and R.H. Scheller, SNAREs - engines for membrane fusion. Nature Reviews Molecular Cell Biology, 2006. 7(9): p. 631-643.

24. Hayashi, T., et al., Synaptic vesicle membrane fusion complex: action of clostridial neurotoxins on assembly. EMBO J, 1994. 13(21): p. 5051-61.

25. Fasshauer, D., et al., Structural changes are associated with soluble Nethylmaleimide-sensitive fusion protein attachment protein receptor complex formation. Journal of Biological Chemistry, 1997. 272(44): p. 28036-28041.

26. Hazzard, J., T.C. Sudhof, and J. Rizo, NMR analysis of the structure of synaptobrevin and of its interaction with syntaxin. J Biomol NMR, 1999. 14(3): p. 203-7.

27. Schuette, C.G., et al., Determinants of liposome fusion mediated by synaptic SNARE proteins. Proceedings of the National Academy of Sciences of the United States of America, 2004. 101(9): p. 2858-2863. 
Bibliography

28. Poirier, M.A., et al., Protease resistance of syntaxin.SNAP-25.VAMP complexes. Implications for assembly and structure. J Biol Chem, 1998. 273(18): p. 11370-7.

29. Fasshauer, D., et al., Identification of a minimal core of the synaptic SNARE complex sufficient for reversible assembly and disassembly. Biochemistry, 1998. 37(29): p. 10354-10362.

30. Margittai, M., et al., Homo- and heterooligomeric SNARE complexes studied by site-directed spin labeling. Journal of Biological Chemistry, 2001. 276(16): p. 13169-13177.

31. Lerman, J.C., et al., Structural analysis of the neuronal SNARE protein syntaxin-1A. Biochemistry, 2000. 39(29): p. 8470-9.

32. Fernandez, I., et al., Three-dimensional structure of an evolutionarily conserved $N$ terminal domain of syntaxin 1A. Cell, 1998. 94(6): p. 841-849.

33. Weimbs, T., et al., A model for structural similarity between different SNARE complexes based on sequence relationships. Trends Cell Biol, 1998. 8(7): p. 260-2.

34. Fasshauer, D., et al., Conserved structural features of the synaptic fusion complex: SNARE proteins reclassified as Q- and R-SNAREs. Proc Natl Acad Sci U S A, 1998. 95(26): p. 15781-6.

35. Bock, J.B., et al., A genomic perspective on membrane compartment organization. Nature, 2001. 409(6822): p. 839-41.

36. Antonin, W., et al., Crystal structure of the endosomal SNARE complex reveals common structural principles of all SNAREs. Nat Struct Biol, 2002. 9(2): p. 107-11.

37. Stein, A., et al., Helical extension of the neuronal SNARE complex into the membrane. Nature, 2009. 460(7254): p. 525-8.

38. Hernandez, J.M., et al., Membrane fusion intermediates via directional and full assembly of the SNARE complex. Science, 2012. 336(6088): p. 1581-4.

39. Misura, K.M.S., R.H. Scheller, and W.I. Weis, Self-association of the H3 region of syntaxin $1 A$ - Implications for intermediates in snare complex assembly. Journal of Biological Chemistry, 2001. 276(16): p. 13273-13282.

40. Misura, K.M.S., et al., Crystal structure and biophysical properties of a complex between the N-terminal SNARE region of SNAP25 and syntaxin 1a. Journal of Biological Chemistry, 2001. 276(44): p. 41301-41309.

41. Zhang, F., et al., The four-helix bundle of the neuronal target membrane SNARE complex is neither disordered in the middle nor uncoiled at the C-terminal region. J Biol Chem, 2002. 277(27): p. 24294-8. 
42. Xiao, W., et al., The neuronal t-SNARE complex is a parallel four-helix bundle. Nat Struct Biol, 2001. 8(4): p. 308-11.

43. Baumert, M., et al., Synaptobrevin: an integral membrane protein of 18,000 daltons present in small synaptic vesicles of rat brain. EMBO J, 1989. 8(2): p. 37984.

44. Scheller, R.H., Membrane trafficking in the presynaptic nerve terminal. Neuron, 1995. 14(5): p. 893-7.

45. Hanson, P.I., J.E. Heuser, and R. Jahn, Neurotransmitter release - four years of SNARE complexes. Curr Opin Neurobiol, 1997. 7(3): p. 310-5.

46. Otto, H., P.I. Hanson, and R. Jahn, Assembly and disassembly of a ternary complex of synaptobrevin, syntaxin, and SNAP-25 in the membrane of synaptic vesicles. Proc Natl Acad Sci U S A, 1997. 94(12): p. 6197-201.

47. Xu, T., et al., Inhibition of SNARE complex assembly differentially affects kinetic components of exocytosis. Cell, 1999. 99(7): p. 713-22.

48. Wiederhold, K. and D. Fasshauer, Is Assembly of the SNARE Complex Enough to Fuel Membrane Fusion? Journal of Biological Chemistry, 2009. 284(19): p. 1314313152.

49. Fasshauer, D., et al., SNARE assembly and disassembly exhibit a pronounced hysteresis. Nat Struct Biol, 2002. 9(2): p. 144-51.

50. Nicholson, K.L., et al., Regulation of SNARE complex assembly by an N-terminal domain of the t-SNARE Sso1p. Nat Struct Biol, 1998. 5(9): p. 793-802.

51. Rice, L.M., P. Brennwald, and A.T. Brunger, Formation of a yeast SNARE complex is accompanied by significant structural changes. FEBS Lett, 1997. 415(1): p. 4955.

52. Fiebig, K.M., et al., Folding intermediates of SNARE complex assembly. Nature Structural Biology, 1999. 6(2): p. 117-123.

53. Demircioglu, F.E., PhD thesis, Comparative studies on regulation of SNARE complex formation by the SM protein Sly1p, University of Goettingen. 2011.

54. Fasshauer, D. and M. Margittai, A transient N-terminal interaction of SNAP-25 and syntaxin nucleates SNARE assembly. Journal of Biological Chemistry, 2004. 279(9): p. 7613-7621.

55. Pobbati, A.V., A. Stein, and D. Fasshauer, $N$ - to C-terminal SNARE complex assembly promotes rapid membrane fusion. Science, 2006. 313(5787): p. 673-6. 
Bibliography

56. Jahn, R. and D. Fasshauer, Molecular machines governing exocytosis of synaptic vesicles. Nature, 2012. 490(7419): p. 201-7.

57. Brenner, S., The genetics of Caenorhabditis elegans. Genetics, 1974. 77(1): p. 7194.

58. Verhage, M., et al., Synaptic assembly of the brain in the absence of neurotransmitter secretion. Science, 2000. 287(5454): p. 864-9.

59. Varoqueaux, F., et al., Total arrest of spontaneous and evoked synaptic transmission but normal synaptogenesis in the absence of Munc13-mediated vesicle priming. Proc Natl Acad Sci U S A, 2002. 99(13): p. 9037-42.

60. Toonen, R.F. and M. Verhage, Munc18-1 in secretion: lonely Munc joins SNARE team and takes control. Trends Neurosci, 2007. 30(11): p. 564-72.

61. Sudhof, T.C. and J.E. Rothman, Membrane fusion: grappling with SNARE and SM proteins. Science, 2009. 323(5913): p. 474-7.

62. Hickey, C.M. and W. Wickner, HOPS initiates vacuole docking by tethering membranes before trans-SNARE complex assembly. Mol Biol Cell, 2010. 21(13): p. 2297-305.

63. Hata, Y., C.A. Slaughter, and T.C. Sudhof, Synaptic vesicle fusion complex contains unc-18 homologue bound to syntaxin. Nature, 1993. 366(6453): p. 347-51.

64. Dulubova, I., et al., A conformational switch in syntaxin during exocytosis: role of munc18. Embo Journal, 1999. 18(16): p. 4372-4382.

65. Misura, K.M.S., R.H. Scheller, and W.I. Weis, Three-dimensional structure of the neuronal-Sec1-syntaxin 1a complex. Nature, 2000. 404(6776): p. 355-362.

66. Burkhardt, P., et al., Munc18a controls SNARE assembly through its interaction with the syntaxin N-peptid. Embo Journal, 2008. 27(7): p. 923-933.

67. Furgason, M.L.M., et al., The N-terminal peptide of the syntaxin Tlg2p modulates binding of its closed conformation to Vps45p. Proceedings of the National Academy of Sciences of the United States of America, 2009. 106(34): p. 1430314308.

68. Khvotchev, M., et al., Dual modes of Munc18-1/SNARE interactions are coupled by functionally critical binding to syntaxin-1 $n$ terminus. Journal of Neuroscience, 2007. 27(45): p. 12147-12155.

69. Rathore, S.S., et al., Syntaxin N-terminal peptide motif is an initiation factor for the assembly of the SNARE-Sec1/Munc18 membrane fusion complex. Proc Natl Acad Sci U S A, 2010. 107(52): p. 22399-406. 
Bibliography

70. Shen, J.S., et al., Selective activation of cognate SNAREpins by Sec1/Munc18 proteins. Cell, 2007. 128(1): p. 183-195.

71. Wojcik, S.M. and N. Brose, Regulation of membrane fusion in synaptic excitationsecretion coupling: speed and accuracy matter. Neuron, 2007. 55(1): p. 11-24.

72. Basu, J., et al., A minimal domain responsible for Munc13 activity. Nat Struct Mol Biol, 2005. 12(11): p. 1017-8.

73. Li, W., et al., The crystal structure of a Munc13 C-terminal module exhibits a remarkable similarity to vesicle tethering factors. Structure, 2011. 19(10): p. 144355.

74. Ma, C., et al., Munc13 mediates the transition from the closed syntaxin-Munc18 complex to the SNARE complex. Nat Struct Mol Biol, 2011. 18(5): p. 542-9.

75. Ma, C., et al., Reconstitution of the Vital Functions of Munc18 and Munc13 in Neurotransmitter Release. Science, 2012.

76. Perin, M.S., et al., Phospholipid binding by a synaptic vesicle protein homologous to the regulatory region of protein kinase C. Nature, 1990. 345(6272): p. 260-3.

77. Pang, Z.P. and T.C. Sudhof, Cell biology of Ca2+-triggered exocytosis. Curr Opin Cell Biol, 2010. 22(4): p. 496-505.

78. Stein, A., et al., Synaptotagmin activates membrane fusion through a Ca2+dependent trans interaction with phospholipids. Nat Struct Mol Biol, 2007. 14(10): p. 904-11.

79. Arac, D., et al., Close membrane-membrane proximity induced by $\mathrm{Ca}(2+)$ dependent multivalent binding of synaptotagmin-1 to phospholipids. Nat Struct Mol Biol, 2006. 13(3): p. 209-17.

80. Martens, S., M.M. Kozlov, and H.T. McMahon, How synaptotagmin promotes membrane fusion. Science, 2007. 316(5828): p. 1205-8.

81. Stein, A. and R. Jahn, Complexins living up to their name--new light on their role in exocytosis. Neuron, 2009. 64(3): p. 295-7.

82. Neher, E., Complexin: does it deserve its name? Neuron, 2010. 68(5): p. 803-6.

83. McMahon, H.T., et al., Complexins: cytosolic proteins that regulate SNAP receptor function. Cell, 1995. 83(1): p. 111-9.

84. Chen, X., et al., Three-dimensional structure of the complexin/SNARE complex. Neuron, 2002. 33(3): p. 397-409. 
Bibliography

85. Giraudo, C.G., et al., A clamping mechanism involved in SNARE-dependent exocytosis. Science, 2006. 313(5787): p. 676-80.

86. Xue, M., et al., Binding of the complexin $N$ terminus to the SNARE complex potentiates synaptic-vesicle fusogenicity. Nat Struct Mol Biol, 2010. 17(5): p. 56875.

87. Yang, X., et al., Complexin clamps asynchronous release by blocking a secondary $\mathrm{Ca}(2+)$ sensor via its accessory alpha helix. Neuron, 2010. 68(5): p. 907-20.

88. Cho, R.W., et al., Genetic analysis of the Complexin trans-clamping model for crosslinking SNARE complexes in vivo. Proc Natl Acad Sci U S A, 2014. 111(28): p. 1031722.

89. Hanson, P.I. and S.W. Whiteheart, $A A A+$ proteins: have engine, will work. Nat Rev Mol Cell Biol, 2005. 6(7): p. 519-29.

90. Whiteheart, S.W., et al., N-ethylmaleimide-sensitive fusion protein: a trimeric ATPase whose hydrolysis of ATP is required for membrane fusion. J Cell Biol, 1994. 126(4): p. 945-54.

91. Nagiec, E.E., A. Bernstein, and S.W. Whiteheart, Each domain of the Nethylmaleimide-sensitive fusion protein contributes to its transport activity. Journal of Biological Chemistry, 1995. 270(49): p. 29182-8.

92. Fleming, K.G., et al., A revised model for the oligomeric state of the Nethylmaleimide-sensitive fusion protein, NSF. J Biol Chem, 1998. 273(25): p. 1567581.

93. May, A.P., et al., Crystal structure of the amino-terminal domain of $\mathrm{N}$ ethylmaleimide-sensitive fusion protein. Nat Cell Biol, 1999. 1(3): p. 175-82.

94. Yu, R.C., R. Jahn, and A.T. Brunger, NSF N-terminal domain crystal structure: models of NSF function. Mol Cell, 1999. 4(1): p. 97-107.

95. Lenzen, C.U., et al., Crystal structure of the hexamerization domain of $\mathrm{N}$ ethylmaleimide-sensitive fusion protein. Cell, 1998. 94(4): p. 525-36.

96. Furst, J., et al., Electron cryomicroscopy structure of $\mathrm{N}$-ethyl maleimide sensitive factor at 11 A resolution. EMBO J, 2003. 22(17): p. 4365-74.

97. Whiteheart, S.W., et al., SNAP family of NSF attachment proteins includes a brainspecific isoform. Nature, 1993. 362(6418): p. 353-5.

98. Rice, L.M. and A.T. Brunger, Crystal structure of the vesicular transport protein Sec17: Implications for SNAP function in SNARE complex disassembly. Molecular Cell, 1999. 4(1): p. 85-95. 
99. Winter, U., X. Chen, and D. Fasshauer, A Conserved Membrane Attachment Site in alpha-SNAP Facilitates N-Ethylmaleimide-sensitive Factor (NSF)-driven SNARE Complex Disassembly. Journal of Biological Chemistry, 2009. 284(46): p. 3181731826.

100. Hohl, T.M., et al., Arrangement of subunits in $20 \mathrm{~S}$ particles consisting of NSF, SNAPs, and SNARE complexes. Mol Cell, 1998. 2(5): p. 539-48.

101. Wimmer, C., et al., Molecular mass, stoichiometry, and assembly of $20 \mathrm{~S}$ particles. Journal of Biological Chemistry, 2001. 276(31): p. 29091-29097.

102. Marz, K.E., J.M. Lauer, and P.I. Hanson, Defining the SNARE complex binding surface of alpha-SNAP - Implications for SNARE complex disassembly. Journal of Biological Chemistry, 2003. 278(29): p. 27000-27008.

103. Chang, L.F., et al., Structural characterization of full-length NSF and 205 particles. Nature Structural \& Molecular Biology, 2012. 19(3): p. 268-U21.

104. Zhao, C., E.C. Smith, and S.W. Whiteheart, Requirements for the catalytic cycle of the N-ethylmaleimide-Sensitive Factor (NSF). Biochim Biophys Acta, 2012. 1823(1): p. 159-71.

105. Cipriano, D.J., et al., Processive ATP-driven substrate disassembly by the Nethylmaleimide-sensitive factor (NSF) molecular machine. J Biol Chem, 2013. 288(32): p. 23436-45.

106. Ryu, J.K.e.a., NSF disassembles a single SNARE complex in one round of ATP turnover. in preparation, 2014.

107. Hayashi, T., et al., Disassembly of the Reconstituted Synaptic Vesicle MembraneFusion Complex in-Vitro. Embo Journal, 1995. 14(10): p. 2317-2325.

108. $\mathrm{Xu}, \mathrm{T} .$, et al., Multiple kinetic components of exocytosis distinguished by neurotoxin sensitivity. Nat Neurosci, 1998. 1(3): p. 192-200.

109. Hua, S.Y. and M.P. Charlton, Activity-dependent changes in partial VAMP complexes during neurotransmitter release. Nat Neurosci, 1999. 2(12): p. 1078-83.

110. Chen, Y.A., S.J. Scales, and R.H. Scheller, Sequential SNARE assembly underlies priming and triggering of exocytosis. Neuron, 2001. 30(1): p. 161-70.

111. Rettig, J. and E. Neher, Emerging roles of presynaptic proteins in Ca++-triggered exocytosis. Science, 2002. 298(5594): p. 781-5.

112. $\mathrm{Xu}, \mathrm{T}$., et al., Early requirement for alpha-SNAP and NSF in the secretory cascade in chromaffin cells. EMBO J, 1999. 18(12): p. 3293-304. 
Bibliography

113. Borisovska, M., et al., v-SNAREs control exocytosis of vesicles from priming to fusion. EMBO J, 2005. 24(12): p. 2114-26.

114. Sorensen, J.B., et al., Sequential $N$ - to C-terminal SNARE complex assembly drives priming and fusion of secretory vesicles. EMBO J, 2006. 25(5): p. 955-66.

115. Walter, A.M., et al., Synaptobrevin N-terminally bound to syntaxin-SNAP-25 defines the primed vesicle state in regulated exocytosis. J Cell Biol, 2010. 188(3): p. 401-13.

116. Wiederhold, K., et al., A coiled coil trigger site is essential for rapid binding of synaptobrevin to the SNARE acceptor complex. J Biol Chem, 2010. 285(28): p. 21549-59.

117. Gao, Y., et al., Single reconstituted neuronal SNARE complexes zipper in three distinct stages. Science, 2012. 337(6100): p. 1340-3.

118. Li, F., et al., A Half-Zippered SNARE Complex Represents a Functional Intermediate in Membrane Fusion. J Am Chem Soc, 2014.

119. Min, D., et al., Mechanical unzipping and rezipping of a single SNARE complex reveals hysteresis as a force-generating mechanism. Nat Commun, 2013. 4: p. 1705.

120. Kiessling, V., et al., Rapid fusion of synaptic vesicles with reconstituted target SNARE membranes. Biophys J, 2013. 104(9): p. 1950-8.

121. Wickner, W., Membrane fusion: five lipids, four SNAREs, three chaperones, two nucleotides, and a Rab, all dancing in a ring on yeast vacuoles. Annu Rev Cell Dev Biol, 2010. 26: p. 115-36.

122. Haas, A., B. Conradt, and W. Wickner, G-protein ligands inhibit in vitro reactions of vacuole inheritance. J Cell Biol, 1994. 126(1): p. 87-97.

123. Jun, Y. and W. Wickner, Assays of vacuole fusion resolve the stages of docking, lipid mixing, and content mixing. Proceedings of the National Academy of Sciences of the United States of America, 2007. 104(32): p. 13010-13015.

124. Reese, C., F. Heise, and A. Mayer, Trans-SNARE pairing can precede a hemifusion intermediate in intracellular membrane fusion. Nature, 2005. 436(7049): p. 410-4.

125. Ungermann, C., et al., A vacuolar v-t-SNARE complex, the predominant form in vivo and on isolated vacuoles, is disassembled and activated for docking and fusion. J Cell Biol, 1998. 140(1): p. 61-9.

126. Ungermann, C., K. Sato, and W. Wickner, Defining the functions of trans-SNARE pairs. Nature, 1998. 396(6711): p. 543-8. 
127. Collins, K.M. and W.T. Wickner, Trans-SNARE complex assembly and yeast vacuole membrane fusion. Proc Natl Acad Sci U S A, 2007. 104(21): p. 8755-60.

128. Starai, V.J., C.M. Hickey, and W. Wickner, HOPS proofreads the trans-SNARE complex for yeast vacuole fusion. Mol Biol Cell, 2008. 19(6): p. 2500-8.

129. Xu, H., et al., HOPS prevents the disassembly of trans-SNARE complexes by Sec17p/Sec18p during membrane fusion. EMBO J, 2010. 29(12): p. 1948-60.

130. Schwartz, M.L. and A.J. Merz, Capture and release of partially zipped trans-SNARE complexes on intact organelles. J Cell Biol, 2009. 185(3): p. 535-49.

131. Weber, T., et al., SNAREpins: Minimal machinery for membrane fusion. Cell, 1998. 92(6): p. 759-772.

132. Mima, J., et al., Reconstituted membrane fusion requires regulatory lipids, SNARES and synergistic SNARE chaperones. EMBO J, 2008. 27(15): p. 2031-42.

133. Weber, T., et al., SNAREpins are functionally resistant to disruption by NSF and alphaSNAP. J Cell Biol, 2000. 149(5): p. 1063-72.

134. Mima, J. and W. Wickner, Complex lipid requirements for SNARE- and SNARE chaperone-dependent membrane fusion. J Biol Chem, 2009. 284(40): p. 27114-22.

135. Stroupe, C., et al., Minimal membrane docking requirements revealed by reconstitution of Rab GTPase-dependent membrane fusion from purified components. Proc Natl Acad Sci U S A, 2009. 106(42): p. 17626-33.

136. Mima, J. and W. Wickner, Phosphoinositides and SNARE chaperones synergistically assemble and remodel SNARE complexes for membrane fusion. Proc Natl Acad Sci U S A, 2009. 106(38): p. 16191-6.

137. Xu, H. and W.T. Wickner, N-terminal domain of vacuolar SNARE Vam7p promotes trans-SNARE complex assembly. Proc Natl Acad Sci U S A, 2012. 109(44): p. 1793641.

138. Zick, M. and W. Wickner, The tethering complex HOPS catalyzes assembly of the soluble SNARE Vam7 into fusogenic trans-SNARE complexes. Mol Biol Cell, 2013. 24(23): p. 3746-53.

139. Siddiqui, T.J., et al., Determinants of synaptobrevin regulation in membranes. Mol Biol Cell, 2007. 18(6): p. 2037-46.

140. Vites, O., E.L. Florin, and R. Jahn, Docking of liposomes to planar surfaces mediated by trans-SNARE complexes. Biophys J, 2008. 95(3): p. 1295-302. 
Bibliography

141. Domanska, M.K., et al., Single vesicle millisecond fusion kinetics reveals number of SNARE complexes optimal for fast SNARE-mediated membrane fusion. J Biol Chem, 2009. 284(46): p. 32158-66.

142. De Blas, G.A., et al., Dynamics of SNARE assembly and disassembly during sperm acrosomal exocytosis. PLoS Biol, 2005. 3(10): p. e323.

143. Park, Y., et al., alpha-SNAP Interferes with the Zippering of the SNARE Protein Membrane Fusion Machinery. J Biol Chem, 2014. 289(23): p. 16326-16335.

144. Fasshauer, D., et al., Mixed and non-cognate SNARE complexes. Characterization of assembly and biophysical properties. J Biol Chem, 1999. 274(22): p. 15440-6.

145. Green, M.R., Sambrook, J., Molecular Cloning: A Laboratory Manual (Fourth Edition). 2012.

146. Froger, A. and J.E. Hall, Transformation of plasmid DNA into E. coli using the heat shock method. J Vis Exp, 2007(6): p. 253.

147. Dower, W.J., J.F. Miller, and C.W. Ragsdale, High efficiency transformation of E. coli by high voltage electroporation. Nucleic Acids Res, 1988. 16(13): p. 6127-45.

148. Schagger, H., Tricine-SDS-PAGE. Nat Protoc, 2006. 1(1): p. 16-22.

149. Hernandez, J.M., PhD thesis, Reconstituted SNARE-mediated fusion: towards a mechanistic understanding, University of Goettingen. 2012.

150. Bradford, M.M., Rapid and Sensitive Method for Quantitation of Microgram Quantities of Protein Utilizing Principle of Protein-Dye Binding. Analytical Biochemistry, 1976. 72(1-2): p. 248-254.

151. Struck, D.K., D. Hoekstra, and R.E. Pagano, Use of resonance energy transfer to monitor membrane fusion. Biochemistry, 1981. 20(14): p. 4093-9.

152. Winter, U., PhD thesis, Biophysical Characterization of SNARE Complex Disassembly Catalyzed by NSF and SNAP, University of Goettingen. 2008.

153. J.R., L., Principles of Fluorescence Spectroscopy. 3rd ed. 2006.

154. Cypionka, A., et al., Discrimination between docking and fusion of liposomes reconstituted with neuronal SNARE-proteins using FCS. Proc Natl Acad Sci U S A, 2009. 106(44): p. 18575-80.

155. Vennekate, W., et al., Cis- and trans-membrane interactions of synaptotagmin-1. Proc Natl Acad Sci U S A, 2012. 109(27): p. 11037-42.

156. Towbin, H., T. Staehelin, and J. Gordon, Immunoblotting in the clinical laboratory. J Clin Chem Clin Biochem, 1989. 27(8): p. 495-501. 
157. Hanson, P.I., et al., The N-ethylmaleimide-sensitive fusion protein and alpha-SNAP induce a conformational change in syntaxin. J Biol Chem, 1995. 270(28): p. 1695561.

158. Li, Y., et al., A single mutation in the recombinant light chain of tetanus toxin abolishes its proteolytic activity and removes the toxicity seen after reconstitution with native heavy chain. Biochemistry, 1994. 33(22): p. 7014-20.

159. Hernandez, J.M., et al., Variable cooperativity in SNARE-mediated membrane fusion. Proc Natl Acad Sci U S A, 2014.

160. McNew, J.A., et al., Close is not enough: SNARE-dependent membrane fusion requires an active mechanism that transduces force to membrane anchors. J Cell Biol, 2000. 150(1): p. 105-17.

161. Barszczewski, M., et al., A novel site of action for alpha-SNAP in the SNARE conformational cycle controlling membrane fusion. Mol Biol Cell, 2008. 19(3): p. 776-84.

162. Kyoung, M., et al., In vitro system capable of differentiating fast Ca2+-triggered content mixing from lipid exchange for mechanistic studies of neurotransmitter release. Proc Natl Acad Sci U S A, 2011. 108(29): p. E304-13.

163. Weninger, K., et al., Accessory proteins stabilize the acceptor complex for synaptobrevin, the 1 : 1 syntaxin/SNAP-25 complex. Structure, 2008. 16(2): p. 308320.

164. Kummel, D., et al., Complexin cross-links prefusion SNAREs into a zigzag array. Nat Struct Mol Biol, 2011. 18(8): p. 927-33.

165. Wei, S., et al., Exocytotic mechanism studied by truncated and zero layer mutants of the C-terminus of SNAP-25. EMBO J, 2000. 19(6): p. 1279-89.

166. Zorman, S., et al., Common intermediates and kinetics, but different energetics, in the assembly of SNARE proteins. Elife, 2014: p. e03348.

167. Helm, C.A., J.N. Israelachvili, and P.M. McGuiggan, Role of hydrophobic forces in bilayer adhesion and fusion. Biochemistry, 1992. 31(6): p. 1794-805.

168. Donaldson, S.H., Jr., et al., General hydrophobic interaction potential for surfactant/lipid bilayers from direct force measurements between light-modulated bilayers. Proc Natl Acad Sci U S A, 2011. 108(38): p. 15699-704.

169. JN, I., Intermolecular and Surface Forces. 3rd Ed. London, San Diego: Academic Press. 2010. 
Bibliography

170. Sakuma, Y., et al., Adhesion of binary giant vesicles containing negative spontaneous curvature lipids induced by phase separation. Eur Phys J E Soft Matter, 2008. 25(4): p. 403-13. 